\title{
Topological string on elliptic CY 3-folds and the ring of Jacobi forms
}

\author{
Min-xin Huang, ${ }^{a}$ Sheldon $\mathrm{Katz}^{b}$ and Albrecht Klemm ${ }^{c}$ \\ ${ }^{a}$ Interdisciplinary Center for Theoretical Study, \\ University of Science and Technology of China, Hefei, Anhui 230026, China \\ ${ }^{b}$ Department of Mathematics, University of Illinois at Urbana-Champaign, \\ 1409 W. Green St., Urbana, IL 6180, U.S.A. \\ ${ }^{c}$ Bethe Center for Theoretical Physics (BCTP), \\ Physikalisches Institut, Universität Bonn, 53115 Bonn, Germany \\ E-mail: minxin@ustc.edu.cn, katz@math.uiuc.edu, \\ aklemm@th.physik.uni-bonn.de
}

ABSTRACT: We give evidence that the all genus amplitudes of topological string theory on compact elliptically fibered Calabi-Yau manifolds can be written in terms of meromorphic Jacobi forms whose weight grows linearly and whose index grows quadratically with the base degree. The denominators of these forms have a simple universal form with the property that the poles of the meromorphic form lie only at torsion points. The modular parameter corresponds to the fibre class while the rôle of the string coupling is played by the elliptic parameter. As a consequence the topological string amplitudes are modular and quasi periodic in the string coupling. This leads to very strong all genus results on these geometries, which are checked against results from curve counting. The structure can be viewed as an indication that an $N=2$ analog of the reciprocal of the Igusa cusp form exists that might govern the topological string theory on these Calabi-Yau manifolds completely.

KeYwords: Topological Strings, String Duality

ArXiv EPrint: 1501.04891 


\section{Contents}

1 Introduction and summary 1

2 The elliptic Calabi-Yau manifolds and their mirrors 4

2.1 Construction of toric hypersurface Calabi-Yau spaces 5

2.2 The $X_{18}(1,1,1,6,9) 3$-fold, an elliptic fibration over $\mathbb{P}^{2} 5$

2.3 Integral symplectic basis and genus zero topological string amplitudes $\quad 7$

3 Involution symmetry and BCOV formalism $\quad 8$

$\begin{array}{ll}3.1 \text { The involution symmetry } & 8\end{array}$

$\begin{array}{ll}3.2 \text { Monodromy group versus involution symmetry } & 10\end{array}$

$\begin{array}{lll}3.2 .1 & \text { The local limit } & 11\end{array}$

$\begin{array}{lll}3.3 & \text { Involution symmetry at genus one } & 12\end{array}$

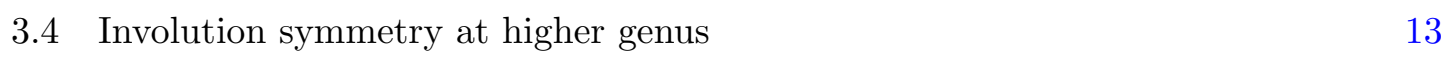

$\begin{array}{lll}\text { 3.4.1 The propagators and rigid special geometry } & 13\end{array}$

$\begin{array}{lll}3.4 .2 & \text { Projective special Kähler manifolds } & 14\end{array}$

$\begin{array}{lll}3.4 .3 & \text { Involution symmetry on the propagators } & 15\end{array}$

$\begin{array}{ll}\text { 3.4.4 Involution symmetry on the higher genus amplitudes } & 16\end{array}$

4 Fiber modularity $\quad 18$

$\begin{array}{ll}4.1 \text { The modular anomaly equation } & 18\end{array}$

4.2 The Hirzebruch surface $\mathbb{F}_{1}$ as base 20

$\begin{array}{ll}4.3 \text { The ring of weak Jacobi forms } & 21\end{array}$

4.4 Weak Jacobi Forms and holomorphic anomaly equation 23

4.5 Exact formulae for base degree zero 24

$\begin{array}{lll}4.6 & \text { Exact formulae for higher base degrees } & 25\end{array}$

$\begin{array}{lll}5 & \text { BPS invariants } & \mathbf{3 2}\end{array}$

5.1 Physical definition of the BPS invariants 32

5.1.1 Unrefined BPS invariants 32

$\begin{array}{lll}\text { 5.1.2 Refined BPS invariants } & 33\end{array}$

5.2 Gopakumar-Vafa invariants from geometry 34

$\begin{array}{lll}\text { 5.2.1 Geometry of curves } & 34\end{array}$

$\begin{array}{lll}\text { 5.2.2 Examples and computations } & 38\end{array}$

$\begin{array}{ll}\text { A Gopakumar-Vafa invariants } & 45\end{array}$

B Derivation of the involution symmetry on the propagators $\quad 47$

C Reducing the ambiguity with the involution symmetry $\quad 52$ 
D Fibre modularity versus involution symmetry

$\begin{array}{lll}\text { D.1 Some (quasi-)modularity formulae } & 58\end{array}$

D.2 Proof of Conjecture 2 and Conjecture 3. 62

$\begin{array}{ll}\text { E Expansion around a point on the conifold divisor } & 70\end{array}$

\section{Introduction and summary}

Topological strings on non-compact Calabi-Yau geometries are solvable and have a very interesting structure with a wealth of connections to gauge theories, integrable models, large N-dualities, Chern-Simons theories, supersymmetric localisation and matrix models. In fact topological string techniques had considerable influence on these areas and solved important problems, see [18] for a recent example. As a benchmark problem in topological string theory with a similar wealth of connections to quantum gravity problems, remains the solution of the topological string on compact Calabi-Yau manifolds. In this paper we report on some progress in this matter.

It follows from the Feynman rules ${ }^{1}$ of [7] that the genus $g$ topological string amplitudes $F_{g}\left(S^{i j}, S^{j}, S ; \underline{z}\right)$ are inhomogeneous polynomials of weighted degree $3 g-3$ in the anholomorphic propagators, $S^{i j}, S^{j}:=S^{j \varphi}, S:=S^{\varphi \varphi}$, which have respective weights $(1,2,3)$, with rational functions of the moduli $\underline{z}$ as coefficients. The holomorphic anomaly determines all of $F_{g}$ except the weight zero piece $f_{g}$, the so called holomophic ambiguity [7] whose determination is the key conceptual obstacle to solving the topological string on compact Calabi-Yau spaces. A careful analysis of the conifold gap condition [44], regularity at the orbifold and Castelnuovo's criterium for the vanishing of higher genus curves reveals that the $f_{g}$ can be determined up genus $51,{ }^{2}$ for the quintic [44]. While for noncompact models the same conditions lead to a complete solution of the closed topological string on these geometries $[29],{ }^{3}$ on compact Calabi-Yau manifolds one needs additional boundary condition.

One purpose of this article is to investigate the additional symmetries and boundary conditions that are specific for elliptic fibered Calabi-Yau threefolds. ${ }^{4}$ Beside the motivation to solve the topological string in general the elliptically fibered cases are very interesting in their own right as the topological string amplitudes predict terms in the low energy

\footnotetext{
${ }^{1}$ See the vertex rule $(6.10)[7]$.

${ }^{2}$ That condition has been derived under the assumption that the ring of modular generators on the quintic has no relations at high weight, which has been checked up to the weight needed for genus 30 .

${ }^{3}$ In fact our key example $X_{18}(1,1,1,6,9)$ is an elliptic CY manifold over $\mathbb{P}^{2}$ and decompactifying the elliptic fibre, leads to a local model solved in [29].

${ }^{4}$ Note that the transitions between Calabi-Yau manifolds [68] and mirror symmetry make it possible to predict from the solution of the topological string on one Calabi-Yau 3 fold with fibration structure the ones of such without fibration structure, if the complex moduli space of the latter is embedded into the one of the former, see [32] for many examples.
} 
effective actions of F-theory and calculate indices of $6 \mathrm{~d}$ conformal theories in particular the $E$-string and its generalizations with gauge theories [28].

One possible constraint could come from the $S L(2, \mathbb{Z})$ modularity of the amplitudes in the Kähler parameter of the elliptic fibre that has been argued in [3,54] based on a monodromy analysis in one global case [12] and calculations for more general elliptic threefolds [52]. It also extends observations for local elliptic surfaces inside Calabi-Yau spaces [55] and [31, 34, 35].

We find that the constraint from the fibre modularity is equivalent to the constraint that comes from a $\mathbb{Z}_{2}$ involution symmetry $I$, that acts on the moduli space of elliptic fibrations as an R symmetry, i.e. it is not realized as a symplectic transformation like the monodromies of the model. This symmetry $I$ acts also on the $F_{g}$ by $(-1)^{(g-1)}$ and on the propagators in a way described in section 3.4.3. Knowing these actions we can restrict the possible constants in the rational ambiguity $f_{g}$ to roughly one fourth. Using this information, the conifold gap and the regularity at the other points in the moduli space, especially the orbifold point we can solve the topological string for all classes to genus 9 on an elliptic fibration over $\mathbb{P}^{2}$, with one section. This is already the strongest available result for compact multiparameter Calabi-Yau manifolds and needs only very mild results on the vanishing of the BPS numbers.

Our main result is however as follows. Consider the topological string partition function $Z=\exp (F)=\exp \left(\sum_{g=0}^{\infty} \lambda^{2 g-2} F_{g}\right)$ expanded in the base degree(s). E.g. for the $\mathbb{P}^{2}$ case one has ${ }^{5}$

$$
Z=Z_{0}\left(1+\sum_{d_{B}=1}^{\infty} Z_{d_{B}}(\tau, z) Q^{d_{B}}\right),
$$

where $\tau$ is the complexified Kähler class of the fiber, and $Q=e^{2 \pi i t_{B}}$ with $t_{d_{B}}$ the suitable defined complexified Kähler class of the base, see (4.1). $Z_{d_{B}}$ is a quotient of even weak Jacobi forms of the following form ${ }^{6}$

$$
Z_{d_{B}}(\tau, z)=\frac{\varphi_{d_{B}}(\tau, z)}{\eta^{36 d_{B}(\tau) \prod_{k=1}^{d_{B}} \varphi_{-2,1}(\tau, k z)}}
$$

where $\eta(\tau)$ is the Dedekind function and $\varphi_{d_{B}}(\tau, z)$ is an even weak Jacobi form of index $\frac{1}{3} d_{B}\left(d_{B}-1\right)\left(d_{B}+4\right)$ and weight $16 d_{B}$. Note that the elliptic argument $z$ is identified by $2 \pi z=\lambda$ with the topological string coupling $\lambda$ and as a consequence the topological string amplitudes are modular (4.15) quasiperiodic (4.16) in the string coupling!

The number of coefficients of $\varphi_{d_{B}}(\tau, z)$ expressed in the basis of the ring of even weak Jacobi forms $Q:=E_{4}(\tau), R:=E_{6}(\tau), A:=\varphi_{-2,1}(\tau, z)$ and $B:=\varphi_{0,1}(\tau, z)$ still grows very fast with $d_{B}^{6}$, however we can argue that the Castelnuovo bounds discussed in section 5.2 bring down the growth to $d_{B}^{4}$. Combined with the restriction from the constraints from the conifold gap and the regularity of the $F_{g}$ in the interior of the moduli space and with

\footnotetext{
${ }^{5}$ The base degree zero contribution $Z_{0}$, is rather trivial and discussed separately.

${ }^{6}$ Here we use the standard notation of the elliptic argument $z$. We trust that there will be no confusion with the B-model moduli, which are also traditionally denoted by $z$.
} 
the independence of these conditions assumed, ${ }^{7}$ this allows us to solve the model up to genus $g=189$ for arbitrary base and fibre classes, or up to $d_{B} \leq 20$ for arbitrary genus and fibre classes.

We will show in section 4.4 that the Jacobi forms satisfy a heat equation form of the holomorphic anomaly equation for $Z_{d_{B}}$ that is equivalent to a limit of the wave function equation proposed by Witten [73] for the topological string partition function in interpreting the holomorphic anomaly equation of [7] as a consequence of a background independent quantization of $H_{3}(M, \mathbb{R})$. This heat equation form of the holomorphic anomaly equation further summarizes all the holomorphic anomaly equations for the fibre modulus, which were proven in [54], i.e. the ansatz (1.2) identically satisfies the holomorphic anomaly equation for the fibre modulus. The relation of the wave function equation and the heat equation was pointed out in [73] and applied to the case in which the moduli space is a symmetric space [26], which however is not the case for generic SU(3) holonomy manifolds.

We argue that the special form of the denominator in our ansatz naturally is crucial to satisfying the Castelnuovo bounds. The scaling of the $z$ argument in the denominator can be interpreted as coming from multiple string windings of the base similar as in the elliptic genus of E-strings [11, 30, 51] or more general strings of 6d SCFTs [28] with gauge symmetries. On the other hand, the paper [51] constructs a $2 \mathrm{~d}$ quiver gauge theory for E-strings and [28] for the $\hat{D}_{4}$ string that can in principle compute the elliptic genus of any finite number of E-strings with the techniques of $[5] .{ }^{8}$

The prediction from the B-model calculations and from (1.1), (1.2) are sucessfully checked in section 5.2 against results from enumerative geometry. Since the moduli space of the BPS states tends to becomes smoother for fixed degree and high $g$ we can make an infinite number of nontrivial checks on the form of $Z_{d_{B}}$ and the determination of its coefficients following the methods proposed in [50].

A remarkable mathematical characterization of the form (1.2) is is that, again due to the form of the denominator, the poles of $Z_{d_{B}}$ lie only at the points $z \in \mathbb{Q} \tau+\mathbb{Q}$, i.e. at torsion points of the elliptic curve. As such they have a modified theta expansion that involves mock modular forms [75]. These structures played a role in the understanding of wall-crossing for $N=4$ BPS states [15] based on the reciprocal of the Igusa cusp form and related meromophic forms.

The general structure (1.1), (1.2) can be viewed as an extension of the only compact case 3 -fold case $K 3 \times T 2$ for which all BPS states associated ${ }^{9}$ to moduli space of curves are known [50]. Due to the SU(2) holonomy the Type II compactification has $N=4$ supersymmetry. The prediction is based on the formula for the elliptic genus of symmetric products of K3 [17] and M-theory/Type IIB duality. It has been recently proven in [66]. In this case the BPS generating function $Z_{B P S}$ is related to the reciprocal of the Igusa

\footnotetext{
${ }^{7}$ This holds at least for as far as we could actually calculate i.e. up to $d_{B} \leq 5, d_{E}, g$ arbitrary or $g \leq 8$, $d_{B}, d_{E}$ arbitrary.

${ }^{8}$ It would be very interesting to see if such $2 \mathrm{~d}$ quiver theory duals exist also for the compact threefold case, i.e. $6 \mathrm{~d}$ theories that do include gravity.

${ }^{9}$ In order to make this interpretation one has to lift zero modes that come from the $T^{2}$ and make the $N=2$ invariants trivially zero.
} 
cusp form of a genus two curve with weight 10 which has a Fourier expansion in $p=$ $\exp (2 \pi i \sigma)$ as $^{10}$

$$
Z_{B P S}(\Omega)=\frac{1}{\Phi_{10}(\Omega)}=\sum_{m=-1} \psi_{-10, m}(\tau, z) p^{m}, \text { with } \Omega=\left(\begin{array}{cc}
\tau & z \\
z & \sigma
\end{array}\right),
$$

where $\Omega$ parametrizes the Siegel upper half space $\mathbb{H}_{2}$ and $\psi_{0, m+1}=\eta^{24}(\tau) \varphi_{-2,1} \psi_{-10, m}$ are meromophic Jacobi-Forms of weight zero and index $m+1$ in the analogeous expansion of $\hat{Z}(\Omega)=\eta^{24} \phi_{-2,1} Z_{B P S}(\Omega)$. The latter can written in terms of the elliptic genera of symmetric products of $K 3$ as

$$
\hat{Z}(\Omega)=\sum_{m=-1} \chi\left(\operatorname{Sym}^{m+1}(K 3), q, y\right) p^{m}=\frac{1}{p} \prod_{\substack{n>0, m \geq 0, l \\ l, m, n \in \mathbb{Z}}} \frac{1}{\left(1-p^{n} q^{m} y^{l}\right)^{c(m \cdot n, l)}}
$$

and summed to the total free energy $F(\lambda, Q)=\log (Z)=\sum_{j=1}^{\infty} \frac{1}{j} F^{(j)}$ by [50]

$$
F^{(j)}=\left(2 \sin \frac{j \lambda}{2}\right)^{-2} \prod_{\substack{k>0, n>0 \\ m \geq 0, l \in \mathbb{Z}}} \frac{\left(1-q^{j k}\right)^{4}}{\left(1-y^{j} q^{j k}\right)^{2}\left(1-y^{-j} q^{j k}\right)^{2}} \frac{1}{\left(1-p^{j n} q^{j m} y^{l j}\right)^{c(m \cdot n, l)}},
$$

where $c(m, r)$ are the expansion coefficients of the elliptic genus of K3 (4.22) and we identified $\lambda=2 \pi z$ and defined $y=\exp (i \lambda)$.

Of course a key question for a full $N=2$ extension of the discussed structure is whether the formulas (1.1), (1.2) come from an underlying modular object as (1.3), (1.5). One obvious difference is the quadratic versus linear growth of the index in the weak Jacobi forms. In section 3.4.2 we discuss the formulation of projective special Kähler manifolds in $N=2$ supergravity that might give a hint for the construction of such an object. We hope to report on progress on that question in [39].

In this article we focus for simplicity on elliptic fibrations with only $I_{1}$ singular fibers in the Kodaira classification, i.e. no gauge symmetries, but eventually flavor symmetries, in the $F$ theory interpretation. We note however that for the gauge theory case, i.e. elliptic fibrations with higher Kodaira fibre singularities, the work [28] gives already the crucial hint for the generalization of the numerator in (1.2). In this case, we will find additional Jacobi theta functions in the numerator, whose different elliptic arguments correspond to the additional Kähler classes of the resolution. This Jacobi theta function will introduce additional zeros in the numerator, which signal the gauge symmetry enhancements, and should be fixed by this structure. The formulas we find also suggest a possible refinement, which we shortly indicate in (5.1.2).

\section{The elliptic Calabi-Yau manifolds and their mirrors}

In this section we describe the toric construction of elliptically fibered Calabi-Yau spaces with only $I_{1}$ Kodaira fibers. Mirror symmetry is manifest in this formalism. We collect the key data of our main example and describe shortly the general structure of the genus zero sector.

\footnotetext{
${ }^{10}$ We follow the notation of [15].
} 


\subsection{Construction of toric hypersurface Calabi-Yau spaces}

Our construction of mirror pairs of Calabi-Yau n-folds as hypersurfaces in toric ambient spaces $\mathbb{P}_{\Delta}^{n+1}$ follows Batyrev's construction which relies on dual pairs of $n+1$ dimensional reflexive pairs of lattice polyhedra $\left(\Delta, \Delta^{*}\right)$. Let $\left(\Gamma, \Gamma^{*}\right)$ be dual lattices with pairing $\langle.,$.$\rangle and$ real completion $\left(\Gamma_{\mathbb{R}}, \Gamma_{\mathbb{R}}^{*}\right)$. A lattice polyhedron $\Delta\left(\Delta^{*}\right)$ is the convex hull of integer points in $\Gamma\left(\Gamma^{*}\right)$. The dual polyhedron $\Delta^{*}$ to $\Delta$ is defined by $\Delta^{*}=\left\{y \in \Gamma_{\mathbb{R}}^{*} \mid\langle y, x\rangle \geq-1, \forall x \in \Delta\right\}$. $\Delta$ is reflexive if $\Delta^{*}$ is a lattice polyhedron. Note $\left(\Delta^{*}\right)^{*}=\Delta$ so if $\Delta$ is reflexive $\left(\Delta, \Delta^{*}\right)$ is a reflexive pair and each polyhedron contains the origin as its unique inner point.

The Calabi-Yau n-fold $M_{n}$ is given by the canonical hypersurface

$$
P=\sum_{\nu_{i} \in \hat{\Delta} \cap \Gamma} a_{i}^{*} \prod_{\nu_{j}^{*} \in \hat{\Delta}^{*} \cap \Gamma^{*}} y_{j}^{\left\langle\nu_{i}, \nu_{j}^{*}\right\rangle+1}=0
$$

in the toric ambient space $\mathbb{P}_{\Delta}$ with coordinate ring $y_{j}$, while the mirror Calabi-Yau n-fold $W_{n}$ is given by the constraint $P^{*}=0$ in $\mathbb{P}_{\Delta^{*}}$ with coordinate ring $x_{i}$, where $P^{*}=0$ is defined analogously to (2.1) with $\Delta$ and $\Delta^{*}$ exchanged. The notation $\hat{\Delta}\left(\hat{\Delta}^{*}\right)$ means the polyhedra $\Delta\left(\Delta^{*}\right)$ with the integer points interior to codimension one faces omitted.

To give the ambient space $\mathbb{P}_{\Delta}$ a fibration structure ${ }^{11}$ such that the embedded CalabiYau $n$-fold defined as hypersurface has a fibration by a Calabi-Yau $m$-fold, we combine a base polyhedron $\Delta^{B *}$ and a reflexive fibre polyhedron $\Delta^{F *}$ into an $n+1$ dimensional polyhedron $\Delta^{*}$ as follows

$$
\left|\begin{array}{cc|cc}
\nu_{i}^{*} \in \Delta^{*} & \multicolumn{2}{c}{\nu_{j} \in \Delta} \\
& \nu_{i}^{F *} & & \nu_{j}^{F} \\
\Delta_{n-m}^{B *} & \vdots & s_{i j} \Delta_{n-m}^{B} & \vdots \\
& \nu_{i}^{F *} & & \nu_{j}^{F} \\
0 \ldots 0 & & 0 \ldots 0 & \\
\vdots & \Delta_{m+1}^{* F} & \vdots & \Delta_{m+1}^{F} \\
0 \ldots 0 & & 0 \ldots 0 &
\end{array}\right| .
$$

If $\Delta^{* F}$ and $\Delta^{* B}$ are reflexive then $\left(\Delta, \Delta^{*}\right)$, given by the complex hull of the indicated points, is a reflexive pair. Reflexivity of $\Delta^{* F}$ is required by the CY condition on the fibre. For $\Delta_{n-m}^{* B}$ it is not a necessary condition, see [43] for more details on this construction. We defined $s_{i j}=\left\langle\nu_{i}^{F}, \nu_{j}^{F *}\right\rangle+1 \in \mathbb{N}$ and scaled $\Delta^{B} \rightarrow s_{i j} \Delta^{B}$. Here we indicated the dimensions of some polyhedra by subscripts; elliptic fibrations correspond to $m=1$.

For $n=3$ and $m=1$ we get many examples by choosing any of the 16 reflexive polyhedra in $2 \mathrm{~d}$ as $\Delta^{* F}$ and $\Delta^{* B}$ respectively and specifying in addition $\nu_{i}^{F *} \in \Delta^{* F}$ as well as twisting parameters [10], which not indicated in (2.2).

\subsection{The $X_{18}(1,1,1,6,9) 3$-fold, an elliptic fibration over $\mathbb{P}^{2}$}

Our main example is the smooth elliptic fibration over $\mathbb{P}^{2}$. This case $\mathrm{e}^{12}$ is a two parameter model discussed in [32] and in greater detail in [12] and illustrates the general construction described above.

\footnotetext{
${ }^{11}$ See exercise in [22] p. 49, where the statement is made in the language of the fans associated to $\Delta$.

${ }^{12}$ It can be also written as the zero locus of a degree 18 polynomial in the weighted projective space $\mathbb{P}^{4}(1,1,1,6,9)$ called $X_{18}(1,1,1,6,9)$.
} 
Pick for the base $\mathbb{P}^{2}$, whose toric polyhedron is the convex hull of the points $\Delta^{* B}=\operatorname{conv}(\{(1,0),(0,1),(-1,-1)\})$, for the fibre polynomial $\Delta^{* F}=$ $\operatorname{conv}(\{(1,0),(0,1),(-2,-3)\})$ and for $\nu_{3}^{* F}=(-2,-3)$. Then $\nu_{3}^{F}=(-1,-1)$ and $s_{33}=6$.

We list the points which give rise to the coordinate ring of $\mathbb{P}=\mathbb{P}_{\Delta} /\left(\mathbb{Z}_{18} \times \mathbb{Z}_{6}\right)$, all points $\nu_{i}^{*} \in \hat{\Delta}^{*}$ and the two vectors of linear relations among them, which correspond to the Mori cone of $\mathbb{P}_{\Delta^{*}}$, as well the toric divisors $D_{x_{i}}=\left\{x_{i}=0\right\}$

\begin{tabular}{|c|rrrr|rrrr|rrr|}
\hline Div. & \multicolumn{1}{c|}{$\nu_{i}$} & \multicolumn{1}{c|}{$\bar{\nu}_{i}^{*}$} & \multicolumn{1}{c|}{$l^{(E)}$} & $l^{(B)}$ \\
\hline$D_{x_{0}}$ & 0 & 0 & 0 & 0 & 0 & 0 & 0 & 0 & -6 & 0 \\
$D_{x_{1}}=L$ & 12 & -6 & -1 & -1 & 1 & 0 & -2 & -3 & 0 & 1 \\
$D_{x_{2}}=L$ & -6 & 12 & -1 & -1 & 0 & 1 & -2 & -3 & 0 & 1 \\
$D_{x_{3}}=L$ & -6 & -6 & -1 & -1 & -1 & -1 & -2 & -3 & 0 & 1 \\
$D_{z}=E$ & 0 & 0 & -1 & -1 & 0 & 0 & -2 & -3 & 1 & -3 \\
$D_{x}=2 H$ & 0 & 0 & 2 & -1 & 0 & 0 & 1 & 0 & 2 & 0 \\
$D_{y}=3 H$ & 0 & 0 & -1 & 1 & 0 & 0 & 0 & 1 & 3 & 0 \\
\hline
\end{tabular}

The classical topological data of the 3 -fold $M$ are easily calculable from the toric construction. The Euler is $\chi(M)=-540$, the two independent Hodge numbers are $h^{1,1}(M)=2$, $h^{2,1}(M)=272$, the classical triple intersection numbers are given by ${ }^{13}$

$$
C_{111}^{0}=H^{3}=9, \quad C_{112}^{0}=H^{2} \cdot L=3, \quad C_{122}^{0}=H \cdot L^{2}=1, \quad C_{222}^{0}=L^{3}=0
$$

where $H$ and $L$ are the divisors dual to the curves defined by the Mori vectors $l^{(E)}$ and $l^{(B)}$ and the Kähler classes $J_{E}$ and $J_{B}$. The intersection with the second Chern class $c_{2}$ of $M$ are

$$
\int_{M} c_{2} \wedge J_{E}=\left[c_{2}\right] \cdot H=36, \quad \int_{M} c_{2} \wedge J_{B}=\left[c_{2}\right] \cdot L=102 .
$$

The mirror manifold is given by the zero locus

$$
P^{*}=x_{0}\left(z^{6}\left(x_{1}^{18}+x_{2}^{18}+x_{3}^{18}-b\left(x_{1} x_{2} x_{3}\right)^{6}\right)-2^{\frac{1}{3}} \sqrt{3} a z x_{1} x_{2} x_{3} x_{4} x_{5}+x^{3}+y^{2}\right)=0
$$

in the space $\mathbb{P} . z:=x_{4}, x:=x_{5}=$ and $y:=x_{6}$ are the conventional names of variables in the Weierstrass form of the elliptic fibre. In $\mathbb{P}$ there are toric $\mathbb{C}^{*}$ actions on the coordinates $x_{i}, i=1, \ldots, 6$, which can be used to eliminate all $a_{i}$, but the two complex structure variables $(a, b)$ of $W$. This is because two $\mathbb{C}^{*}$ actions

$$
x_{i} \rightarrow \mu_{r}^{l_{i}^{(r)}} x_{i}, \text { with } \mu_{r} \in \mathbb{C}^{*},
$$

are divided out from the coordinate ring of $\mathbb{P}$. One can introduce manifestly $\mathbb{C}^{*}$ invariant combinations the $a_{i}$ as complex structure variables of $W$, namely

$$
z_{i}=(-1)^{l_{0}^{(i)}} \prod_{k=1} a_{k}^{l_{k}^{(i)}}, \quad i=1, \ldots, h_{21}\left(W_{3}\right)=h_{11}\left(M_{3}\right) .
$$

\footnotetext{
${ }^{13}$ In the notation of [32] these intersections are encoded in the ring $\mathcal{R}=9 J_{E}^{3}+3 J_{E}^{2} J_{B}+J_{E} J_{B}^{2} . H, L$ are the notations for the divisors used in [12].
} 
In the case at hand $z_{1}:=z_{E}=\frac{a_{4} a_{5}^{2} a_{6}^{3}}{a_{0}^{6}}$ corresponds to the elliptic fibre and $z_{2}:=z_{B}=\frac{a_{1} a_{2} a_{3}}{a_{4}^{3}}$ to the base class. Using the $\mathbb{C}^{*}$ actions on the period integrals $\Pi(z)=\int_{\gamma_{3}} \Omega$ with $\left(a=a_{0}\right)$

$$
\Omega=\oint_{\gamma_{\epsilon}} \frac{a \mu}{P^{*}}
$$

given by a residuum integral around $P^{*}=0$ with the measure $\mu=\sum_{i=1}^{5}(-1)^{i} w_{i} d \tilde{x}_{1} \wedge$ $\ldots \widehat{d x}_{i} \ldots \wedge d \tilde{x}_{5}$, one can derive two Picard-Fuchs (PF) differential equations [32]

$$
\begin{aligned}
& \mathcal{L}_{1}=\theta_{1}\left(\theta_{1}-3 \theta_{2}\right)-12 z_{1}\left(6 \theta_{1}+1\right)\left(6 \theta_{1}+5\right), \\
& \mathcal{L}_{2}=\theta_{2}^{3}+z_{2} \prod_{i=0}^{2}\left(3 \theta_{2}-\theta_{1}+i\right)
\end{aligned}
$$

determining the periods from $\mathcal{L}_{i} \Pi(z)=0, i=1,2$. Here $\theta_{i}=z_{i} \frac{\partial}{\partial z_{i}}$. The discriminants of the operators are

$$
\begin{aligned}
& \Delta_{1}=\left(1-432 z_{1}\right)^{3}-27 z_{2}\left(432 z_{1}\right)^{3}, \\
& \Delta_{2}=1+27 z_{2}
\end{aligned}
$$

The 3-point couplings can be computed from the PF operators [32]

$$
\begin{array}{ll}
C_{111}=\frac{9}{z_{1}^{3} \Delta_{1}}, & C_{112}=C_{121}=C_{211}=\frac{3 \Delta}{z_{1}^{2} z_{2}} \\
C_{122}=C_{212}=C_{221}=\frac{\Delta_{3}^{2}}{z_{1} z_{2}^{2} \Delta_{1}}, & C_{222}=\frac{9\left(\Delta_{3}^{3}+\left(432 z_{1}\right)^{3}\right)}{z_{2}^{2} \Delta_{1} \Delta_{2}},
\end{array}
$$

where for convenience we can define the factor $\Delta_{3}=1-432 z_{1}$.

\subsection{Integral symplectic basis and genus zero topological string amplitudes}

The PF operators determine the 3 -point couplings. Its solutions in a special basis determine the metric on the moduli space, which is a Kähler manifold $G_{i \bar{\jmath}}=\partial_{t_{i}} \partial_{t_{\bar{j}}} K$, whose Kähler potential $K$ is given by

$$
e^{-K}=i \int_{W} \Omega \wedge \bar{\Omega}=i \Pi^{\dagger} \eta \Pi
$$

where $\left(k=h^{1,1}(W)+1\right)$

$$
\eta=\left(\begin{array}{cc}
0 & \mathbf{1}_{k \times k} \\
-\mathbf{1}_{k \times k} & 0
\end{array}\right) .
$$

is the symplectic pairing on $H_{3}(W, \mathbb{Z})$ and $\Pi(z)=\left(X^{I}=\int_{A^{I}} \Omega, F_{I}=\int_{B_{I}} \Omega\right)^{T}$ is the period vector w.r.t. to the corresponding symplectic basis $\left(A^{I}, B_{I}\right), I=0, \ldots, k-1$ of $H_{3}(W, \mathbb{Z})$. The two structures are related by special geometry, which implies the existence of a prepotential, the genus zero amplitude $\mathcal{F}^{(0)}$, with

$$
\Pi=\left(\begin{array}{c}
X^{0} \\
X^{i} \\
F_{0} \\
F_{i}
\end{array}\right)=X^{0}\left(\begin{array}{c}
1 \\
t^{i} \\
2 \mathcal{F}^{(0)}-t^{i} \partial_{i} \mathcal{F}^{(0)} \\
\frac{\partial \mathcal{F}^{(0)}}{\partial t^{i}}
\end{array}\right)=X^{0}\left(\begin{array}{c}
1 \\
t^{i} \\
-\frac{C_{i j k}^{0}}{3 !} t^{i} t^{j} t^{k}+\frac{\int c_{2} \wedge J_{i}}{24} t^{i}-i \frac{\chi \zeta(3)}{(2 \pi)^{3}}+f(q) \\
\frac{C_{i j k}^{0}}{2} t^{i} t^{j}+n_{i j} t^{j}+\frac{\int c_{2} \wedge J_{i}}{24}+\partial_{i} f(\underline{q})
\end{array}\right) .
$$


Here

$$
\mathcal{F}^{(0)}=\left[-\frac{C_{i j k} t^{i} t^{j} t^{k}}{3 !}+n_{i j} \frac{t^{i} t^{j}}{2}+\frac{\int c_{2} \wedge J_{i}}{24} t^{i}-i \frac{\chi \zeta(3)}{2(2 \pi)^{3}}+f(\underline{q})\right]
$$

and $X^{I}$ are homogeneous coordinates and $t^{i}(z)$ are inhomogeneous coordinates

$$
t^{i}(z)=\frac{X^{i}(\underline{z})}{X^{0}(\underline{z})}=\frac{1}{2 \pi i}\left(\log \left(z_{i}\right)+\Sigma^{i}(\underline{z})\right), \quad i=1, \ldots, h^{1,1}(W),
$$

which serve as a mirror map. In particular the complexified volumes of the curves in the Mori cone $t_{i}$ are the flat coordinates at large radius near $z_{i}=0, \forall i=1, \ldots, h^{11}(M)$, where the third equal sign in (2.15) is valid, $X^{0}$ is the unique holomorphic period normalized to $\omega_{0}(z)=1+\mathcal{O}(z)$. The $C_{i j k}^{(0)}$ are the classical intersection numbers. There is a freedom in choosing the $n_{i j}$, but because of the odd intersections and the requirement of integer monodromy around $z_{i}=0$, they cannot be set to zero as they must be half integral. Following [12] or the topological description the quadratic terms in $\mathcal{F}^{(0)}$ in the appendix of [44] we may take them to $n_{11}=\frac{9}{2}$ and $n_{12}=\frac{3}{2}$ for the $X_{18}(1,1,1,6,9)$ model. Like for all toric hypersurfaces in this example the Picard-Fuchs equations are of the generalized hyperelliptic type and all logarithmic solutions can be derived from

$$
\omega_{0}(\underline{z}, \underline{\rho})=\sum_{\underline{n}} c(\underline{n}, \underline{\rho}) z^{\underline{n}+\underline{\rho}} \text { where } c(\underline{n}, \underline{\rho})=\frac{\prod_{j} \Gamma\left(\sum_{\alpha} l_{0 j}^{(\alpha)}\left(n_{\alpha}+\rho_{\alpha}\right)+1\right)}{\prod_{i} \Gamma\left(\sum_{\alpha} l_{i}^{(\alpha)}\left(n_{\alpha}+\rho_{\alpha}\right)+1\right)},
$$

with $X^{0}(\underline{z})=\omega_{0}(\underline{z}, 0)$, by taking derivatives with respect to $\rho_{\alpha}$, in particular

$$
X^{i}(\underline{z})=\left.\frac{\partial_{\rho_{i}}}{2 \pi i} \omega_{0}(\underline{z}, \underline{\rho})\right|_{\underline{\rho}=\underline{0}},
$$

see the appendix in [33] for the explicit structure of the higher derivatives.

\section{Involution symmetry and BCOV formalism}

In this section we mainly discuss the involution symmetry, its realization on the topological string amplitudes and its consequences. In the course of the exposition we also review the relevant aspects of the BCOV formalism. Many of the actual calculations are quite technical and relegated to the appendices. We comment on the relation of the involution symmetry to the monodromies in subsection 3.2 and on the local limit in subsection 3.2.1. The section 3.4.1 illustrates the idea of the BCOV ring and contains many modular properties needed in section 4. Subsection 3.4.2 is more speculative and tries to give a perspective on a possible global modular object related to the all genus amplitude.

\subsection{The involution symmetry}

Fibrations with fibre polytope $\Delta^{* F}$ have an involution symmetry $I$ acting on the moduli space, which is independent of the chosen base $B$. The action of $I$ on the $X_{18}(1,1,1,6,9)$ moduli space that we describe below appeared in [12].

$I$ acts non-trivial on the higher genus amplitudes $F_{g}\left(S^{i j}, S^{i}, S\right)$ and the anholomorphic propagators $S^{i j}, S^{j}, S$. As mentioned in the introduction it follows from the Feynman rules 
of [7] that the $F_{g}$ are inhomogeneous polynomials of degree $3 g-3$ with weights $1,2,3$ and rational functions in the moduli as coefficients. Moreover the structure of the genus zero three-point vertex within these Feynman rules implies, that the degree of the denominators of these rational functions grows generically with $\alpha(2 g-2)$. Since the amplitudes are finite for large moduli values this is also an upper bound for degree of the numerators, which turns out to be nearly saturated. This implies that for an $r$ parameter model the complexity of the $F_{g}$ grows ${ }^{14}$ growth very fast with $g^{(r+1)(r+2) / 2+r}$.

In particular the rational function $f_{g}$ that does not multiply any propagator is not determined by the holomorphic anomaly equation, that otherwise fix the $F_{g}$ recursively in $g$. The function $f_{g}$ is restricted by the pole behavior of the $F_{g}$ at the boundary divisors of the moduli space to contain finite number of unknown parameters which growths with $g^{r}$. This is called the holomorphic ambiguity and its determination is a main problem to solve the topological string on compact Calabi-Yau manifolds. If we know the action of $I$ on $F_{g}$ and the propagators we can further restrict $f_{g}$. In fact this requirement cuts down the possible parameters in $f_{g}$ to roughly one fourth. Moreover we find that the symmetry $I$ is equivalent to the constraints that the fibre modularity imposes on $f_{g}$.

To see the involution symmetry explicitly define the monomial $m=\left(\prod_{i} x_{i}\right)^{6}$, where $x_{i}$ are the coordinates of the base. Then

$$
P^{*}=g\left(z, \underline{x}, \underline{a}_{B}\right)-b z^{6} m^{6}-2^{\frac{1}{3}} \sqrt{3} a z m x y+x^{3}+y^{2}=0,
$$

where $g\left(z, \underline{x}, \underline{a}_{B}\right)$ is a polynomial compatible with the scaling, which does not contain $m$. Now requiring that

$$
\begin{aligned}
& x \rightarrow x+c_{1} z^{2} m^{2} \\
& y \rightarrow y+c_{2} x z m+c_{3} z^{3} m^{3}
\end{aligned}
$$

leaves $P^{*}$ invariant fixes $c_{1}=2^{\frac{1}{3}} a^{2}, c_{2}=\frac{(1-i)}{2^{\frac{1}{3}}} \sqrt{3} a$ and $c_{3}=\sqrt{3} a^{3}$ and acts on the parameters as

$$
I:(a, b) \rightarrow\left(i a, b+a^{6}\right) .
$$

This involution operation acts on the $(3,0)$ form $\Omega$ given in $(2.9)$ by

$$
I: \Omega \rightarrow i \Omega
$$

because $P^{*}$ as well as the measure $\mu$ are invariant. Since $\Omega$ defines vacuum line bundle $\mathcal{L}$ and the higher genus amplitudes $\mathcal{F}_{g}$ transforms as section $\mathcal{F}_{g} \in \mathcal{L}^{2 g-2}$ we conclude that the involution symmetry maps $\mathcal{F}^{(g)}$ to $\tilde{\mathcal{F}}^{(g)}$ with

$$
\tilde{\mathcal{F}}^{(g)}=(-1)^{g-1} \mathcal{F}^{(g)} \text {. }
$$

In the the $z_{i}$ coordinates the involution acts as

$$
I: \quad\left(z_{1}, z_{2}\right) \rightarrow\left(x_{1}, x_{2}\right)=\left(\frac{1}{432}-z_{1},-\frac{\left(432 z_{1}\right)^{3} z_{2}}{\left(1-432 z_{1}\right)^{3}}\right) .
$$

\footnotetext{
${ }^{14}$ This estimate is in good agreement with the byte count for the $F_{g}$ in the two parameter model, which occupy $6.5 \times 10^{4}, 1.3 \times 10^{6}, 1.1 \times 10^{7}, 6 \times 10^{7}, 2.4 \times 10^{8}, 7.8 \times 10^{8}, 2.2 \times 10^{9}, 5.5 \times 10^{9}$ bytes of memory for $g=2, \ldots, 9$.
} 
The PF operators (2.10) are invariant up to some trivial factors under the involution. On the other hand, the involution exchanges the two discriminants up to some factors as

$$
I\left(\Delta_{1}\right)=\left(432 z_{1}\right)^{3} \Delta_{2}, \quad I\left(\Delta_{2}\right)=\frac{\Delta_{1}}{\left(1-432 z_{1}\right)^{3}} .
$$

\subsection{Monodromy group versus involution symmetry}

The involution symmetry multiplies the periods w.r.t. $\Omega$ with $i$, so it is in particular not a symplectic transformation (in fact $M_{I}^{T} \eta M_{I}=-\eta$ ) and cannot be related to an actual monodromy action.

The monodromy group is most quickly described as follows. There are Neveu-Schwarz B-field shifts that leave the instanton action invariant $t_{i} \rightarrow t_{i}+1, i=1, \ldots, h^{1,1}(M)$. Their monodromy is completely fixed by (2.15) after specifying the topological data as in subsections 2.2, 2.3 for the main example. We call these monodromies and the corresponding $6 \times 6$ matrices $T_{1}\left(=T_{E}\right)$ and $T_{2}\left(=T_{B}\right)$.

Further there is a cycle $\nu_{1}$, which corresponds to the $B_{3} \sim S^{3}$ base of the StromingerYau-Zaslow $T^{3} \rightarrow B_{3}$ fibration [70], that vanishes at the conifold $\Delta_{1}=0$. In the $X_{6}(1,2,3)$ elliptic fibrations such as the $X_{18}(1,1,1,6,9)$ one has a second conifold discriminant $\Delta_{2}=0$, where $\Delta_{1}$ and are exchanged, up to irrelevant factors, by the involution symmetry as discussed in the last section. Let us call the corresponding vanishing cycle $\nu_{2}$ and use the integral symplectic basis of cycles that corresponds to the period vector (2.15). Using some basic analytic continuation one calculates the vanishing cycles in this basis as ${ }^{15}$

$$
\begin{aligned}
& \nu_{1}=(0,0,0,1,0,0), \\
& \nu_{2}=(-1,0,0,0,1,-3) \text {. }
\end{aligned}
$$

The Lefschetz monodromy theorem for 3-folds states that the monodromy around a conifold divisor, where by definition an $S^{3}$ sphere $\nu$ vanishes, on each cycle $\gamma \in H_{3}(M, \mathbb{Z})$ is given by the symplectic reflection

$$
S_{\nu}(\gamma)=\gamma-\langle\gamma, \nu\rangle \nu
$$

where $\langle$,$\rangle denotes the symplectic pairing. By this formula and (3.8) we can calculate$ the monodromy in the basis (2.15). We call the corresponding monodromies and $6 \times 6$ matrices $C_{1}$ and $C_{2}$ respectively. The $T_{i}$ and $C_{i}$ generate the monodromy group of the $X_{18}(1,1,1,6,9)$ model in fact redundantly, as can be seen by the Van Kampen relations analyzed for this case in great detail in [12]. In particular one has that an order 18 element $A$ is given by ${ }^{16}$

$$
A^{-1}=C_{1} C_{2} T_{2}
$$

and the two conifold monodromies $C_{1}$ and $C_{2}$ are conjugated to each other by the order 6 element $A^{3}$

$$
C_{1}=A^{3} C_{2} A^{-3},
$$

\footnotetext{
${ }^{15}$ In fact $\nu_{1}$ corresponds always to $F_{0}$, while $\nu_{2}$ corresponds to $F_{E}-X_{0}-\sum_{i=1}^{h^{11}(B)} a^{i} F_{B, i}$, where the $a_{i}$ defined above (4.1) .

${ }^{16}$ We use the same notation $A$ for this element as in [12]. The other notions ate related by $C_{1}=T$, $C_{2}=B, T_{1}=T_{\infty}$ and $T_{2}=D_{\infty}$.
} 
which exchanges $\nu_{1} \leftrightarrow \nu_{2}$ and is explicitly

$$
A^{3}=\left(\begin{array}{cccccc}
1 & 1 & 0 & 0 & 0 & 0 \\
-1 & 0 & 0 & 0 & 0 & 0 \\
3 & 3 & 1 & 0 & 0 & -1 \\
-1 & 0 & 0 & 0 & 1 & -3 \\
0 & 10 & 3 & -1 & 1 & -3 \\
0 & 3 & 1 & 0 & 0 & 0
\end{array}\right)
$$

One can conclude that the monodromy group is generated by $A$ and $C_{1}$ [12]. Note that $T_{1}$ and $A^{3}$ acts on $t_{1}$ as [12]

$$
T_{1}: t_{1} \mapsto t_{1}+1, \quad A^{3}: t_{1} \mapsto-\frac{1}{t_{1}+1}
$$

These operations generate an $\mathrm{Sl}(2, \mathbb{Z})$ action on the elliptic fibre parameter, which we call therefore often $\tau$ in the following. As we mentioned already invariance under the involution symmetry $I$ is equivalent to the fibre modularity. This could be guessed from the fact that it is $A^{3}$ that really imposes the nontrivial part of fibre modularity — the shift symmetry is present for all Kähler moduli — and conjugates the conifold monodromies just as $I$ exchanges the two conifold discriminants. The equivalence of the restrictions imposed the by invariance under $I$ and the fibre modularity will be strictly proven in appendix D.

\subsubsection{The local limit}

As we have seen there is an $\operatorname{SL}(2, \mathbb{Z})$ action on the elliptic fibre modulus. This symmetry governs the amplitudes order by order in the exponential $Q_{B}=\exp \left(2 \pi i t_{B}\right)$ of the flat coordinate representing the base curve in $\mathbb{P}^{2}$. There is an other action of the congruence subgroup $\Gamma_{0}(3)=\left\{\left(\begin{array}{ll}a & b \\ c & d\end{array}\right) \in \mathrm{SL}(2, \mathbb{Z}) \mid c=0 \bmod 3\right\}$ of $\mathrm{SL}(2, \mathbb{Z})$ on the base modulus. To see it we must make the volume of the fibre large, $\lim \left(t_{E}\right) \rightarrow i \infty$, which corresponds to $z_{E} \sim q=\exp \left(2 \pi i t_{E}\right) \sim z_{E} \sim 0$. This is known as the local limit. In the $A$-model language it focuses on the $\mathcal{O}(-3) \rightarrow \mathbb{P}^{2}$ geometry by decompactifying the elliptic fibre of $X_{18}(1,1,1,6,9)$. The periods of the local geometry are given by integrals of a meromorphic differential $\lambda$ over cycles on an elliptic curve $\mathcal{C}_{B}$ that is the the mirror geometry. These periods $\Pi_{l o c}=\left(\int_{i} \lambda, i=0, a, b,\right)=\left(1, t_{B},-3 \partial_{t_{B}} F_{l o c}^{(0)}\right)$ fulfill local rigid special geometry [38]. The occurrence of the 1 indicates that the CY $(3,0)$ form $\Omega$ becomes in the local limit the meromorphic one form $\lambda$ which has a non-vanishing residuum at a pole $\lambda$ on $\mathcal{C}_{B}$. From (2.10) one sees that the periods in the local limit $\Pi_{l o c}$ are governed by a specialization of the second differential operator $\mathcal{L}=\theta_{2}^{3}+z_{2} \prod_{i=0}^{2}\left(3 \theta_{2}+i\right)$, which is another way to see the constant solution.

However we want to understand precisely, which periods $\Pi=\int_{\underline{\underline{\Gamma}}} \Omega$ on the compact CY 3-fold become the local periods in the limit or equivalently how the $\Gamma_{0}(3)$ action is embedded in the action of $\operatorname{Sp}(6, \mathbb{Z})$. To see this notice that we have to make a linear 
change in the $\partial_{t_{i}} F^{(0)}, i=1,2$ part of the basis $\Pi$ (2.15) to keep with

$$
\begin{aligned}
& \tilde{F}_{1}=F_{B}=-\frac{3}{2} t_{E}^{2}-t_{E} t_{B}+\frac{3 t_{E}}{2}+\frac{3}{2} \\
& \tilde{F}_{2}=3 F_{B}-F_{E}=\frac{t_{B}^{2}}{2}-\frac{3 t_{B}}{2}+\frac{1}{4}
\end{aligned}
$$

in the limit $z_{E}=0$ three fine periods $\left(X_{0}=1, t_{B}, \tilde{F}_{2}\right)$. If we now conjugate the monodromies that we found in the last section by the corresponding element $C=\left(\begin{array}{ccc}\mathbf{1}_{4 \times 4} & 0 & 0 \\ 0 & 0 & 1 \\ 0 & -1 & 3\end{array}\right)$, we obtain the following monodromies $M_{i}=C T_{2} C^{-1}, M_{c}=C C_{2} C^{-1}$ and $M_{o}=\left(M_{c} M_{i}\right)^{-1}$

$$
M_{i}=\left(\begin{array}{cccccc}
\underline{1} & 0 & 0 & 0 & 0 & 0 \\
0 & 1 & 0 & 0 & 0 & 0 \\
\underline{1} & 0 & 1 & 0 & 0 & \mathbf{0} \\
3 & 2 & 0 & 1 & -1 & 0 \\
0 & -1 & 0 & 0 & 1 & 0 \\
-\underline{1} & 0 & 1 & 0 & 0 & \mathbf{1}
\end{array}\right), M_{c}=\left(\begin{array}{cccccc}
\underline{1} & 0 & 0 & 0 & 0 & 0 \\
1 & 1 & 0 & 0 & 0 & 1 \\
-\underline{3} & 0 & 1 & 0 & 0 & -\mathbf{3} \\
1 & 0 & 0 & 1 & 0 & 1 \\
0 & 0 & 0 & 0 & 1 & 0 \\
\underline{0} & 0 & 0 & 0 & 0 & \mathbf{1}
\end{array}\right), M_{o}=\left(\begin{array}{cccccc}
\underline{1} & 0 & 0 & 0 & 0 & 0 \\
-1 & 1 & 0 & 0 & 0 & -1 \\
2 & 0 & \mathbf{1} & 0 & 0 & \mathbf{3} \\
-3 & -1 & 0 & 1 & 1 & 0 \\
-1 & 1 & 0 & 0 & 1 & -1 \\
-\underline{1} & 0 & -\mathbf{1} & 0 & 0 & -\mathbf{2}
\end{array}\right)
$$

Here we have printed the elements $\left(\begin{array}{ll}d & c \\ a & b\end{array}\right)$ of the $\Gamma_{0}(3)$ subgroup, see [1], in bold face and underlined the shifts due to the non-vanishing residua of $\lambda$. A consequence of this symmetry is the fact that in the strict local limit the amplitudes $F^{(g)}\left(\hat{E}_{2}\left(\tau_{B}\right), G_{2}\left(\tau_{B}\right), G_{4}\left(\tau_{B}\right), G_{6}\left(\tau_{B}\right)\right)[1]$, with $\tau_{B}=\frac{-3 \partial_{u} \partial_{t_{B}} F^{(0)}}{\partial_{u} t_{B}}$, can be expressed in the indicated generators of the ring of almost holomorphic forms of $\Gamma_{0}(3)$ and has been completely solved [29]. Here $u$ is the complex modulus of the local geometry [29], which is identified with the $b$ parameter in (2.6). Of course this base modularity that holds in the strict large fibre limit, and acts not directly on the periods of the 3-fold geometry, extends much less trivially to the whole two parameter family than the fibre modularity, that we discuss further in section 4 .

\subsection{Involution symmetry at genus one}

First we consider the genus one amplitude $\mathcal{F}^{(1)}$ which fulfills the holomorphic anomaly equation $[7]^{17}$

$$
\partial_{i} \bar{\partial}_{\bar{\jmath}} F^{(1)}=\frac{1}{2} \operatorname{Tr} C_{i} \bar{C}_{\bar{\jmath}}-\frac{\chi(M)}{24} G_{i \bar{\jmath}},
$$

where $\left\langle j\left|\bar{\phi}_{\bar{\imath}}\right| k\right\rangle=\bar{C}_{\bar{\imath} \bar{\jmath} \bar{k}}=C_{\bar{\imath}}^{j^{\prime} k^{\prime}} \eta_{j^{\prime} j} \eta_{k k^{\prime}}$ and $\left\langle j\left|\phi_{i}\right| k\right\rangle=C_{i j k}$ are the 3 point functions discussed above, with the relation $C_{\bar{\imath}}^{j k}=e^{2 K} G^{j \bar{\jmath}} G^{k \bar{k}} \bar{C}_{\bar{\imath} \bar{\jmath} \bar{k}}$. The solution has the well known form

$$
\mathcal{F}^{(1)}=\frac{1}{2}\left(3+h^{1,1}-\frac{\chi}{12}\right) K+\frac{1}{2} \log \operatorname{det} G^{-1}-\frac{1}{24} \sum_{i=1}^{2} s_{i} \log z_{i}-\frac{1}{12} \sum_{a=1}^{2} \log \Delta_{a} .
$$

The topological data for the $X_{18}(1,1,1,6,9)$ model are given in section 2.2. The leading asymptotic of $\mathcal{F}^{(1)}$ anholomorphic near large volume limit is

$$
\mathcal{F}^{(1)}=-\frac{1}{24} \sum_{i=1}^{2} t_{i} \int_{M} c_{2} J_{i}+\mathcal{O}(Q) .
$$

\footnotetext{
${ }^{17}$ This was derived first in [6]. We use however the conventions of [7].
} 
The values of the second Chern-Classes (2.5) determine the constants $s_{1}=114, s_{2}=48$. In the holomorphic limit, the determinant of the Kahler metric transforms as $\operatorname{det}(\tilde{G})=$ $\operatorname{det}\left(\frac{\partial z_{i}}{\partial x_{j}}\right) \operatorname{det}(G)$. A simple calculation shows that the invariance of the genus one amplitude $\mathcal{F}^{(1)}$ under the involution transformation (3.6) impose the constrain on the constants $s_{1}=$ $-30+3 s_{2}$, which is consistent with the evaluation of the second Chern class on $J_{i}$.

\subsection{Involution symmetry at higher genus}

The higher genus amplitudes are defined recursively from the holomorphic anomaly equations [7]

$$
\partial_{\bar{\imath}} F^{(g)}=\frac{1}{2} \bar{C}_{\bar{\imath}}^{k l}\left(D_{k} D_{l} F^{(g-1)}+\sum_{r=1}^{g-1} D_{k} F^{(r)} D_{l} F^{(g-r)}\right) .
$$

The $F^{(g)}$ can be integrated using an anholomorphic potential $S$ for $\bar{C}_{\bar{l}}^{j k}$, whose existence is consequence of special geometry or more generally the $t t^{*}$ geometry [7]. One has

$$
\partial_{\bar{\imath}} S=G_{\bar{\imath} j} S^{j}, \quad \partial_{\bar{\imath}} S^{j}=G_{\bar{\imath} k} S^{j k}, \quad \partial_{\bar{\imath}} S^{i j}=\bar{C}_{\bar{\imath}}^{i j} .
$$

The $F^{(g)}$ were obtained in [7] either using the special geometry commutator $\left[D_{i}, \partial_{\bar{\jmath}}\right]_{k}^{l}=$ $G_{k} \delta_{i}^{l}+G_{i \bar{\jmath}} \delta_{k}^{l}-C_{i k n} \bar{C}_{\bar{\jmath}}^{n l}$ and partial integration or a Feynman graph expansion of a master functional, which fulfills a heat equation type of equation. The most vantage point of view is to find directly a solution for this master integral, which we achieve at least iteratively in the base modulus.

\subsubsection{The propagators and rigid special geometry}

It was shown in [74] as a consequence of special geometry that the covariant derivatives $D_{i}$ in (3.19) close on anholomorphic generators, that are closely related to the propagators, up to rational functions of $\underline{z}$. We call the corresponding ring generated by these anholomorphic generators over the rational functions in $\underline{z}$ the BCOV ring.

This principal structure of the BCOV ring can be understood by the analogy to the ring of almost holomorphic forms $\bigoplus_{k} \widehat{\mathcal{M}}_{k}\left(\Gamma_{0}\right)=\mathbb{C}\left[\hat{E}_{2}, E_{4}, E_{6}\right]$ of $\Gamma_{0}=\operatorname{SL}(2, \mathbb{Z})$ (or congruence subgroups thereof) that is graded by the weight $k$. Let us denote the modular transformation $\tau \mapsto \tau_{\gamma}=\frac{a \tau+b}{c \tau+d}$ with $\left(\begin{array}{ll}a & b \\ c & d\end{array}\right) \in \mathrm{SL}(2, \mathbb{Z})$. For $k \in 2 \mathbb{N}_{+}$the normalized Eisenstein series $E_{k}$ of modular weight are defined as [9]

$$
E_{k}(\tau)=\frac{1}{2 \zeta(k)} \sum_{\substack{m, n \in \mathbb{Z} \\(m, n) \neq(0,0)}} \frac{1}{(m \tau+n)^{k}}=1+\frac{(2 \pi i)^{k}}{(k-1) ! \zeta(k)} \sum_{n=1}^{\infty} \sigma_{k-1}(n) q^{n},
$$

where the last equal sign holds straightforwardly only for $k>2$. Here $\sigma_{k}(n)$ is the sum of the $k$-th power of the positive divisors of $n$ and $\zeta(k)=\sum_{r \geq 0} \frac{1}{r^{k}}$ with

$$
\zeta(2 k)=-\frac{(2 \pi i)^{2 k} B_{2 k}}{4 k(2 k-1) !} .
$$

The Bernoulli numbers $B_{k} k \neq 1$ are defined by the generating function

$$
\sum_{m=0}^{\infty} \frac{B_{m} x^{m}}{m !}:=\frac{x}{e^{x}-1} .
$$


If the sum in (3.21) converges $k>2$ it is obvious that $E_{k}\left(\tau_{\gamma}\right)=(c \tau+d)^{k} E_{k}(\tau)$ transforms as a weight $k$ form under modular transformations. For $k=2$ the second equal sign in (3.21) can be viewed as a regularization of the sum on the right [9]. In this case the modular transformation is broken to

$$
E_{2}\left(\tau_{\gamma}\right)=(c \tau+d)^{2} E_{2}(\tau)-\frac{6 i}{\pi} c(c \tau+d) .
$$

Since $\frac{1}{\operatorname{Im}\left(\tau_{\gamma}\right)}=\frac{(c \tau+d)^{2}}{\operatorname{Im}(\tau)}-2 i c(c \tau+d)=\frac{|c \tau+d|^{2}}{\operatorname{Im}(\tau)}$ one can define an almost holomorphic object

$$
\hat{E}_{2}(\tau)=E_{2}(\tau)-\frac{3}{\pi \operatorname{Im}(\tau)},
$$

which transforms as a modular form of weight 2 .

In the solution of the holomorphic anomaly equation $\hat{E}_{2}(\tau)$ plays the rôle of the propagator and the Maass derivative $D_{\tau}: \hat{\mathcal{M}}_{k} \rightarrow \hat{\mathcal{M}}_{k+2}$

$$
D_{\tau} f_{k}=\left(d_{\tau}-\frac{k}{4 \pi \operatorname{Im}(\tau)}\right) f_{k}
$$

on weight $k$ forms, with $d_{\tau}=\frac{1}{2 \pi i} \frac{\mathrm{d}}{\mathrm{d} \tau}$, plays the role of the covariant derivative $D_{i}$. The an-holomorphic version of the Ramanujan identities

$$
D_{\tau} \hat{E}_{2}=\frac{1}{12}\left(\hat{E}^{2}-E_{4}\right), \quad D_{\tau} \hat{E}_{4}=\frac{1}{3}\left(\hat{E}_{2} E_{4}-E_{6}\right), \quad D_{\tau} \hat{E}_{6}=\frac{1}{2}\left(\hat{E}_{2} E_{6}-E_{4}^{2}\right),
$$

show that it closes on $\bigoplus_{k} \widehat{\mathcal{M}}_{k}\left(\Gamma_{0}\right)$. Note that by the isomorphism ([48] Prop. 1) of the ring $\bigoplus_{k} \widehat{\mathcal{M}}_{k}\left(\Gamma_{0}\right)$ and the one quasi modular forms $\bigoplus_{k} \tilde{\mathcal{M}}_{k}\left(\Gamma_{0}\right)=\mathbb{C}\left[E_{2}, E_{4}, E_{6}\right]$ one can take the holomorphic limit in (3.27) by replacing $\hat{E}_{2} \rightarrow E_{2}$ and $D_{\tau} \rightarrow d_{\tau}$ without losing information about the ring structure.

The relation between the BCOV ring of the $X_{18}(1,1,1,6,9)$ Calabi-Yau manifold and the ring of almost holomorphic forms is more then an analogy. In the limit of large fibre discussed in section 3.2.1 the special Kähler structure of supergravity reduces to local rigid special geometry and the BCOV ring of $X_{18}(1,1,1,6,9)$ reduces to $\bigoplus_{k} \widehat{\mathcal{M}}_{k}\left(\Gamma_{0}(3)\right)=$ $\mathbb{C}\left[\hat{E}_{2}, G_{2}, G_{4}, G_{6}\right][1]$.

In the limit of large base the relevant ring becomes $\bigoplus_{k} \widehat{\mathcal{M}}_{k}\left(\Gamma_{0}\right)$ and the structure of the holomorphic anomaly equation can be be even more neatly combined into the ring of weak Jacobi forms, which include all powers of the topological string coupling as we will discuss in section 4 .

\subsubsection{Projective special Kähler manifolds}

The form (1.5) suggests that the all genus amplitude in $K 3 \times T 2$ is related to a modular form associated to a degenerate Riemann surface of genus 3 . In this section we recall some structure of projective special Kähler manifolds, see [21] for a modern review, that relates for $\mathrm{N}=2$ compactifications of type II string the complex structure moduli of Calabi-Yau 3 folds to a family of Riemann surfaces of genus $h_{21}+1$.

Of course we could first address the simpler question, how the objects of rigid special Kähler geometry described in the last section extend to the moduli space of Riemann 
surfaces of higher genus. However this is done in [56], with the result that a holomorphic analog of $E_{2}$ does not exist, but a meromorphic one does.

In the formalism of projective special Kähler manifolds the formal description of the formulae for the propagators and the closing of the covariant derivatives on the propagators, i.e the analogs of the relations (3.27), (B.2), as well as the recursive integration w.r.t. to the anholomorphic generators all simplify relative to the formalism [74] and resemble closely the formalism in the rigid case [25].

However the price one has to pay is a very complicated anholomorphic embedding of the complex moduli space of the Calabi-Yau manifold including the string coupling $\lambda$, that is related to the projective scaling, into the Siegel upper half plane $\mathbb{H}_{h_{21}+1}$ of Riemann surfaces of genus $h_{21}+1$, which is given by [16]

$$
\mathcal{N}_{I J}=\bar{F}_{I J}+2 i \frac{\operatorname{Im}\left(F_{I L}\right) X^{L} \operatorname{Im}\left(F_{J K}\right) X^{K}}{\operatorname{Im}\left(F_{K L}\right) X^{K} X^{L}},
$$

see [62] for a concise review. ${ }^{18}$ Here upper case letters refer to the projective coordinates of the complex moduli space given by a set of $A$-cycle periods $X^{I}(\underline{z}), I=0, \ldots, h_{21}(W)$ in the integral symplectic basis discussed in section 2.3. $F_{I J}(\underline{z})=\frac{\partial^{2} F}{\partial X^{I} \partial X^{J}}$ with $F(\underline{z})=\left(X^{0}\right)^{2} \mathcal{F}^{(0)}$ the prepotential in homogeneous coordinates. The positivity, or rather negativity in the supergravity conventions $\operatorname{Im}\left(\mathcal{N}_{I J}\right)<0$, is discussed in [72]. It is physically enforced by the condition that kinetic terms in vector multiplets and the gauge kinetic terms $\mathcal{L}=$ $-\frac{1}{4} g_{I J}^{-2} F_{n m}^{I} F^{J} n m-\frac{\theta_{I J}}{32 \pi^{2}} F^{I} \tilde{F}^{J}+\ldots$ of the graviphoton $F_{n m}^{0}$ and the vector bosons $F_{n m}^{i}$, $i=1, \ldots, h_{21}$ in vector multiplets with $g_{I J}^{-2}=\frac{i}{4}\left(\mathcal{N}_{I J}-\overline{\mathcal{N}}_{I J}\right)$ and $\theta_{I J}=2 \pi^{2}\left(\mathcal{N}_{I J}+\overline{\mathcal{N}}_{I J}\right)$ must be positive definite.

Note that $\mathcal{N}_{I J}$ transforms under real or integer symplectic transformations, w.r.t. to $(2.14)$

$$
\left(\begin{array}{c}
X^{\prime A} \\
F_{A}^{\prime}
\end{array}\right)=\left(\begin{array}{cc}
D & B \\
B & A
\end{array}\right)\left(\begin{array}{c}
X^{A} \\
F_{A}
\end{array}\right),
$$

with $A^{T} C=C^{T} A, B^{T} D=D^{T} B$ and $A^{T} D-C^{T} B=1$, such as the monodromies discussed in section 3.2 , in the canonical way

$$
\mathcal{N}^{\prime}=(A \mathcal{N}+B)(C \mathcal{N}+D)^{-1} .
$$

This suggests that the special monodromy family of Riemann surface of genus 3 and a better understanding of the map (3.28) might be essential to understand whether the formulae (1.1), (1.2) come from an underlying modular object as in the $N=4$ case.

\subsubsection{Involution symmetry on the propagators}

For the concrete calculation we follow [74] and work in the in-homogenous coordinates and use the BCOV propagators with a shift by derivatives of the Kähler potential as the generators [2]. We denote the propagators after this shift (B.1) as $S^{i j}, S^{i}, S$. The definition of the propagators involves a choice of rational functions in the moduli $\underline{z}[7,52]$, which is

\footnotetext{
${ }^{18}$ We would like to thank Jan Louis for a guidance to the literature.
} 
not unique. It has to be accompanied by a consistent choice of rational functions of $\underline{z}$ in the closing relations (B.2) [2] that generalize (3.27). The derivation of these rational functions for non-hypergeometric, but more interesting Apery like Picard-Fuchs equations that arise in non-abelian gauged linear $\sigma$ models requires a minimal choice that uses the properties of differential equations [46]. For the $X_{18}(1,1,1,6,9)$ model, we summarize two canonical choices and the derivation of the action under the involution symmetry in the appendix B.

Let us now state the action of the involution symmetry on the propagators. Suppose $f\left(z_{1}, z_{2}\right)$ is a rational function of $z_{1}, z_{2}$, we denote with the tilde symbol $\tilde{f}=f\left(x_{1}, x_{2}\right)$, the transformed function by replacing the $z_{i}$ 's in the arguments with $x_{i}$ 's in the involution (3.6). The $\tilde{f}$ can be considered as a function of either $x_{i}$ 's or $z_{i}$ 's using the transformation (3.6). For example under the involution, the three point functions (2.12) transform as a tensor except for a minus sign due to (3.5)

$$
\tilde{C}_{i j k}=-\frac{\partial z_{l}}{\partial x_{i}} \frac{\partial z_{m}}{\partial x_{j}} \frac{\partial z_{n}}{\partial x_{k}} C_{l m n}
$$

We find in B that the action of the involution on the propagators has the following form

$$
\tilde{S}^{i j}=-\frac{\partial x_{i}}{\partial z_{k}} \frac{\partial x_{j}}{\partial z_{m}} S^{k m}, \quad \tilde{S}^{i}=-\frac{\partial x_{i}}{\partial z_{k}} S^{k}+f^{i}, \quad \tilde{S}=-S+f^{0},
$$

The precise form of the rational functions $f_{i}, f_{0}$ depends on the choice of the rational functions in the choice of the propagator as well as in (B.2). Fixing this choice as in (B.3) [2] we find the solution

$$
\begin{aligned}
& f^{1}=\frac{5 z_{1} z_{2}\left(1-1296 z_{1}+559872 z_{1}^{2}\right)}{12\left(1-432 z_{1}\right)^{2}}, \\
& f^{2}=\frac{3732480 z_{1}^{3} z_{2}\left[1-1296 z_{1}+559872 z_{1}^{2}-80621568 z_{1}^{3}\left(1+27 z_{2}\right)\right]}{\left(1-432 z_{1}\right)^{6}} \\
& f^{0}=5\left[45 z_{2}-67+1296 z_{1}\left(67-135 z_{2}\right)-559872 z_{1}^{2}\left(67-315 z_{2}\right)\right. \\
& \left.+80621568 z_{1}^{3}\left(67-630 z_{2}\right)\right] /\left(23328\left(1-432 z_{1}\right)^{3}\right) .
\end{aligned}
$$

\subsubsection{Involution symmetry on the higher genus amplitudes}

The topological string amplitudes transform under the involution symmetry (3.6) by replacing the propagators with the transformed propagators (3.32) and the complex structure coordinates $z_{i}$ 's with $x_{i}$ 's. We would like to understand how the transformed amplitude is related to the original amplitude and how many constraints the symmetry implies.

At higher genus $g \geq 2$, the BCOV holomorphic anomaly equation [7] can be written as partial derivatives with respect to the an-holomorphic generators [2, 25, 74]. This is another way to see the polynomial structure of the amplitudes in the an-holormophic generators and more importantly to obtain them by direct integration in an alogarithm that grows polynomial in complexity in $g$. Assuming the algebraic independence of $K_{i}$ 's, we can write the partial derivatives in terms of lower genus amplitudes. Here the $K_{i}$ appear as in the covariant derivative of higher genus amplitude $D_{i} \mathcal{F}^{(g)}=\partial_{i} \mathcal{F}^{(g)}-(2 g-2) K_{i} \mathcal{F}^{(g)}$. We need 
to be a little careful for the genus one case, as the $K_{i}$ already appear at the ordinary derivative

$$
\partial_{i} \mathcal{F}^{(1)}=\frac{1}{2} C_{i j k} S^{j k}-\left(\frac{\chi}{24}-1\right) K_{i}-\frac{1}{2} s_{i j}^{j}-\partial_{i}\left(\frac{1}{24} \sum_{k=1}^{2} s_{k} \log z_{k}+\frac{1}{12} \sum_{a=1}^{2} \log \Delta_{a}\right),
$$

where we have computed the derivative of Kahler metric in terms of Christoffel connections and use the first equation in (B.2). The equations for partial derivative in the multi moduli case have been worked out in [2]

$$
\begin{aligned}
\frac{\partial \mathcal{F}^{(g)}}{\partial S^{i j}}= & \frac{1}{2} \partial_{i}\left(\partial_{j}^{\prime} \mathcal{F}^{(g-1)}\right)+\frac{1}{2}\left(C_{i j l} S^{l k}-s_{i j}^{k}\right) \partial_{k}^{\prime} \mathcal{F}^{(g-1)}+\frac{1}{2}\left(C_{i j k} S^{k}-h_{i j}\right) c_{g-1} \\
& +\frac{1}{2} \sum_{h=1}^{g-1} \partial_{i}^{\prime} \mathcal{F}^{(h)} \partial_{j}^{\prime} \mathcal{F}^{(g-h)}, \\
\frac{\partial \mathcal{F}^{(g)}}{\partial S^{i}}= & (2 g-3) \partial_{i}^{\prime} \mathcal{F}^{(g-1)}+\sum_{h=1}^{g-1} c_{h} \partial_{i}^{\prime} \mathcal{F}^{(g-h)}, \\
\frac{\partial \mathcal{F}^{(g)}}{\partial S}= & (2 g-3) c_{g-1}+\sum_{h=1}^{g-1} c_{h} c_{g-h},
\end{aligned}
$$

where the $c_{g}$ is defined as

$$
c_{g}= \begin{cases}\frac{\chi}{24}-1, & g=1 \\ (2 g-2) \mathcal{F}^{(g)}, & g>1\end{cases}
$$

We have also used the notation $\partial^{\prime}$ to denote

$$
\partial_{i}^{\prime} \mathcal{F}^{(g)}= \begin{cases}\partial_{i} \mathcal{F}^{(g)}+\left(\frac{\chi}{24}-1\right) K_{i}, & g=1 \\ \partial_{i} \mathcal{F}^{(g)} & g>1\end{cases}
$$

i.e. on the right hand in (3.35), we use the formula (3.34) for $\partial_{i} \mathcal{F}^{(1)}$ omitting the $-\left(\frac{\chi}{24}-1\right) K_{i}$ term.

Since the propagators are symmetric $S^{i j}=S^{j i}$, we can choose to use only the $S^{i j}$ with $i \leq j$. In the case of $i \neq j$, the right hand side of first equation in (3.35) need to be multiplied by an extra factor of 2 to take account of the double contribution.

Let us make some remarks about (3.5). When we combine the amplitude with its involution transformation as in (3.5), we find the dependence on the propagators $S^{i j}, S^{i}, S$ cancels with our proposal for the transformed propagators (3.32) and the shifts (3.33), which is a consistency check of the formulae (3.32), (3.33).

To make an ansatz for the holomorphic ambiguity $f^{(g)}$ at genus $g$, one considers a rational function of $z_{1}, z_{2}$ with a pole of $\left(\Delta_{1} \Delta_{2}\right)^{2 g-2}$. When we combine the correct holomorphic ambiguity with its transformation $\tilde{f}^{(g)}+(-1)^{g} f^{(g)}$ as in (3.5), we find the degree of the pole at discriminant $\Delta_{1} \Delta_{2}$ is reduced for $g>2$, and the pole completely cancels 
for the genus $g=2$ case. Meanwhile, a new pole at $\Delta_{3}=1-432 z_{1}$ appears due to the involution transformation (3.6)..$^{19}$

The use of the involution symmetry greatly reduce the number of unknown constants in the holomorphic ambiguity. In the naive ansatz in [3], the holomorphic ambiguity has the form $\frac{p^{(g)}\left(z_{1}, z_{2}\right)}{\left(\Delta_{1} \Delta_{2}\right)^{2 g-2}}$, where $p^{(g)}\left(z_{1}, z_{2}\right)$ is a polynomial of degree $7(g-1)$ in $z_{1}$ and degree $5(g-1)$ in $z_{2}$. So naively there are $(7 g-6)(5 g-4)$ unknown constants. For example, at genus 2 and 3 , the naive ansatz has 48 and 165 unknown constants. We find the equation (3.5) imposes 36 and 125 conditions on the naive ansatz, so we are left with only 12 and 40 unknown constants to be fixed by boundary conditions. We present a detailed analysis on the reduction of unknowns in appendix C. Together with the conifold gap discussed in appendix E, this allows us to fix the ambiguity to genus 9 .

\section{Fiber modularity}

In this section we reformulate the holomorphic anomaly equation expressing the fibre modularity [54] in a convenient way that allows us to summarize the structure in terms of even weak Jacobi forms. We exemplify the geometry setting with the elliptic fibration over $\mathbb{F}_{1}$, which exhibits geometric limits that yields the BPS numbers the $K 3$ fibre, the BPS numbers on an embedded $\frac{1}{2} K 3$ and maybe most remarkably by blowing down the $\frac{1}{2} K 3$, the compact elliptic fibration over $\mathbb{P}^{2}$. The even weak Jacobi forms are hence a unified mathematical language to address heterotic type duality [63], the six dimensional superconformal theories $[23,55]$ and compact elliptic Calabi-Yau spaces.

\subsection{The modular anomaly equation}

In the simplest case the base $B_{n-1}$ of the elliptic fibration is Fano (or almost Fano),${ }^{20}$ the degenerate elliptic fibers are only of Kodaira type $I_{1}$ and the elliptic fibre has only one holomorphic section and no rational sections. In the language of $F$-theory the complex structure is so generic that the 12-2n dimensional theory is completely higgsed.

The cohomology and the C.T.C. Wall data of $M_{3}$ is then fixed by the cohomology of the base and the fibre type [54]. One key feature of topological string amplitudes that comes from the described geometrical setting is the $\Gamma_{F} \in \mathrm{SL}(2, \mathbb{Z})$ modularity of the amplitudes. Here $\Gamma_{F}$ is the modular group acting projectively on the fibre modulus. In particular for one holomorphic section $\Gamma_{F}$ is the full ${ }^{21} \mathrm{SL}(2, \mathbb{Z})$. In this case the $b_{2}\left(M_{3}\right)$ Kähler classes of $M_{3}$ split in $b_{2}\left(B_{n-1}\right)$ and one class, which corresponds to the elliptic fibre. Let $\tilde{t}_{i}$ be the complexified volumes of the curve classes $[C]_{i}, i=1, \ldots, b_{2}\left(B_{2}\right)$ in the base and $\tau$ the complexified volume of the elliptic fibre. One defines with $q=\exp (2 \pi i \tau)$ a parameter, which becomes exponentially small, when the volume $\operatorname{Im}(\tau)$ of the elliptic fibre becomes large and similarly $\tilde{Q}^{\beta} \equiv \prod_{\alpha=1}^{h_{2}\left(B_{n}\right)} \exp \left(2 \pi i \tilde{t}^{\alpha} \beta_{\alpha}\right)$, with $\beta \in H_{2}\left(B_{n}, \mathbb{Z}\right)$. Let $a^{i}$ be

\footnotetext{
${ }^{19}$ The genus two and three holomorphic ambiguity formulae in [3] miss a constant $\frac{511}{144}$ and $-\frac{2105053}{1959552}$.

${ }^{20}$ To construct already many concrete examples we may assume that $B_{n-1}$ is a toric variety $\mathbb{P}_{\Delta_{n-1}^{* B}}$, i.e. specified by an $n-1$ dimensional reflexive polyhedron $\Delta_{n-1}^{* B}$.

${ }^{21}$ It gets restricted to congruence subgroup $\Gamma_{F} \in \mathrm{SL}(2, \mathbb{Z})$ if the fibration has more rational sections or multi sections.
} 
the intersections of the curves $[C]_{i}$ with the canonical class $K_{B}$ of the base. E.g. for $B_{2}$ a $\mathbb{P}^{2}$ one has $a^{1}=3$, while for $B_{2}$ the Hirzebruch surfaces $\mathbb{F}_{n}$ one has $\left(a^{1}, a^{2}\right)=(2,2-n)$ etc, see [54] for the other toric bases. In order to make the $\mathrm{SL}(2, \mathbb{Z})$ duality in the fibre direction more manifest it is convenient to redefine

$$
t^{i}=\tilde{t}^{i}+\frac{\tau a^{i}}{2},
$$

so that the base moduli are summarized in $Q^{\beta} \equiv \prod_{\alpha=1}^{h_{2}\left(B_{n}\right)} \exp \left(2 \pi i t^{\alpha} \beta_{\alpha}\right)$.

We can write an expansion of the disconnected topological string amplitudes also known as the free energy in the large volume limit

$$
F(\tau, \lambda, Q)=\operatorname{class}(\underline{t})+\sum_{g=0}^{\infty} \lambda^{2 g-2} F^{(g)}(\tau, Q) .
$$

The classical terms

$$
\operatorname{class}(\underline{t})=\lambda^{-2} c_{3}(\underline{t})+c_{1}(\underline{t})
$$

are at genus zero a cubic polynomial $c_{3}(\underline{t})$ in the Kähler parameter $t$ that can be read from (2.16) without the constant term. Similarly $c_{1}(\underline{t})$ can be read from (3.18). Constant terms are proportional to the Euler number and are included via the 0 in the sum over $H_{2}\left(B_{n}, \mathbb{Z}\right)$ in (4.4). This a formal expansion in the string coupling $\lambda$, but each genus $g$ amplitude $F^{(g)}(\tau, Q)$ has a finite radius of convergence in the $(q, Q)$ parameters, determined by the discriminant of the Picard-Fuchs equations. We decompose the genus $g$ amplitudes in fibre $q$ and base moduli $Q$ as

$$
F^{(g)}(\tau, Q)=\sum_{\beta \in H_{2}\left(B_{n}, \mathbb{Z}\right)} F_{\beta}^{(g)}(\tau) Q^{\beta} .
$$

There are two closely related properties partially characterizing the $F_{\beta}^{(g)}(q)$.

- The $F_{\beta}^{(g)}(\tau)$ are, up to a potential sign transformation, meromorphic modular forms in $q$ of weight $2 g-2$. More precisely the $F_{\beta}^{(g)}(\tau)$ can be decomposed into

$$
F_{\beta}^{(g)}(\tau)=Z_{\beta}^{\mathrm{osc}}(\tau) P_{\beta}^{(g)}(\tau) .
$$

The first factor $Z_{\beta}^{\text {osc }}(\tau)$ is the partition function of

$$
n(\beta)=12 \sum_{i} \beta_{i} a^{i}
$$

bosons, i.e. given by $Z_{\beta}^{o s c}=\frac{1}{\eta^{n(\beta)}}$. Here $i=1, \ldots, h_{2}\left(B_{n-1}\right)$. Since $n(\beta) / 12$ can be an odd integer (4.5) can take an additional sign under modular transformations.

The second factor $P_{\beta}^{(g)}(\tau)$ is a polynomial in the generators of almost holomorphic modular forms with respect to the subgroup $\Gamma_{F}$ of $\mathrm{SL}(2, \mathbb{Z})$. In particular for our main examples with the $X_{6}(1,2,3)$ elliptic fibre type $\Gamma_{F}=\mathrm{SL}(2, \mathbb{Z})$ and the generators are given by almost holomorphic weight 2 Eisenstein series $\hat{E}_{2}$ and the holomorphic 
Eisenstein series $E_{4}$ and $E_{6}$. The $w$ weight and hence the degree of the polynomial $P_{\beta}^{(g)}(q)$ grow linearly with $g$ and $\beta$ as

$$
w=2 g+6 \sum_{i} \beta_{i} a^{i}-2
$$

- They satisfy a holomorphic anomaly equation, which for elliptic fibrations one section and only $I_{1}$ fibers is completely determined by topological data of the base ${ }^{22}[3,54]$

$$
\frac{\partial F_{\beta}^{(g)}(\tau)}{\partial \hat{E}_{2}}=\frac{(-1)^{n+1}}{24}\left(\sum_{h=0}^{g} \sum_{\beta^{\prime}+\beta^{\prime \prime}=\beta}\left(\beta^{\prime} \cdot \beta^{\prime \prime}\right) F_{\beta^{\prime}}^{(h)} F_{\beta^{\prime \prime}}^{(g-h)}+\beta \cdot\left(\beta-K_{B}\right) F_{\beta}^{(g-1)}\right) .
$$

In particular $n$ is the dimension of the base, ${ }^{23} K_{B}$ is the canonical class of the base and the dot $\cdot$ is the intersection form on the base. $Z_{\beta}^{o s c}$ can be factored out and (4.8) holds equivalently for $P_{\beta}^{(g)}$. Clearly (4.8) can determine the $P_{\beta}^{(g)}$ only up to a polynomial $P_{\beta}^{(g), h o l}$, which depends only on the holomorphic generators of $\Gamma_{F}$, i.e. in particular not on $\hat{E}_{2}$, and is called the holomorphic or modular ambiguity.

- We define a similar split expansion in fibre and base degree for the partition function $Z=\exp (F(\tau, \lambda, Q))$

$$
Z=1+\sum_{\beta \in H_{2}\left(B_{2}, \mathbb{Z}\right)} Z_{\beta}(\tau, \lambda) Q^{\beta}
$$

We identify now $\lambda=z$ with the elliptic parameter of the weak Jacobi forms ${ }^{24}$ and assign modular weight -1 to it so that $Z$ and $Z_{\beta}(\tau, \lambda)$ have modular weight zero. From (4.8) follows

$$
\left(\partial_{\hat{E}_{2}}+\frac{\beta \cdot\left(\beta-K_{B}\right)}{24} z^{2}\right) Z_{\beta}(\tau, z)=0
$$

Basic properties of the weak Jacobi forms are summarized in the next section, see equation (4.26), yield then that the index of $Z_{\beta}(\tau, z)$ is $\frac{\beta \cdot\left(\beta-K_{B}\right)}{2}$.

As a corollary to the holomorphic anomaly equation (4.8) we have the following. If the base has itself a fibration $B_{n-2} \cdot F=1$ and $F^{2}=0$, we can decompactify the fibre and consider the topological string on the local geometry of a rational fibration over the $B_{n-2}$.

\subsection{The Hirzebruch surface $\mathbb{F}_{1}$ as base}

In this section we present the base $\mathbb{F}_{\alpha}=\mathbb{F}_{1}$ as an explicit example and discuss the relation between the holomorphic anomaly equation in various dimensions. Our base has two divisor classes a minus $-\alpha$ curve which we call $S$ and the fibre class $F$. The canonical class of the base is $K_{B}=2 S+(2+\alpha) F$. One has the intersections $S^{2}=-\alpha, F S=1$ and $F^{2}=0$. We

\footnotetext{
${ }^{22} \mathrm{~A}$ similar form of the holomorphic anomaly equation appeared for rational elliptic surfaces in [34] and for a compact 3-fold with an elliptic and a Enriques fibration in [25].

${ }^{23}$ Since only one dimension was considered the alternating factor was absorbed in the definitions in [54]. Here we exhibit it to specialize to from the threefold to the surface case.

${ }^{24}$ We also use the standard arguments of these forms $(\tau, z)$.
} 
denote $\beta=m F+n S$ and a short form $F_{m F+n S}^{(g)}=F_{m, n}^{(g)}$ so that our anomaly formula (4.8) reads

$$
\begin{aligned}
\frac{\partial F_{m, n}^{(g)}(q)}{\partial \hat{E}_{2}}=-\frac{1}{24}\left(\sum_{h=0}^{g}\right. & \sum_{k=0}^{m} \sum_{l=0}^{n}[k(n-l)+l(m-k)-\alpha l(n-l)] F_{k, l}^{(h)} F_{m-k, n-l}^{(g-h)} \\
+ & {\left.[2 m(n-1)-n(\alpha(n-1)+2)] F_{m, n}^{(g-1)}\right) . }
\end{aligned}
$$

This formula has interesting specialization, e.g. for the $\alpha=1$ case we get

- In the $n=0$ case corresponds to the corollary mentioned above. The two dimensional surface is a specially polarized $K 3$. In this case we get a very simple recursion in the genus

$$
\frac{\partial F_{m}^{(g)}(q)}{\partial \hat{E}_{2}}=-\frac{m}{12} F_{m}^{(g-1)}
$$

but no recursion in the base degree. An all genus result can be obtained for all $m$ using the heterotic one loop result.

- The $m=0$ case corresponds likewise to the corollary. The rational elliptic surface is the $\frac{1}{2} K 3$. The solution has been discussed first in [55] and in the context of the (refined) holomorphic anomaly equation in [11,30,43]. A quiver description has been found in [51].

- $m=n$ corresponds to the blow down of the Hirzebruch surface $\mathbb{F}_{1}$ to $\mathbb{P}^{2}$. For this geometry we get hence

$$
\frac{\partial F_{m}^{(g)}(q)}{\partial \hat{E}_{2}}=-\frac{1}{24}\left(\sum_{h=0}^{g} \sum_{k=0}^{m}[k(m-k)] F_{k}^{(h)} F_{m-k}^{(g-h)}+[m(m-3)] F_{m}^{(g-1)}\right) .
$$

The solution will be discussed in section 4.6.

\subsection{The ring of weak Jacobi forms}

Jacobi forms $\varphi: \mathbb{H} \times \mathbb{C} \rightarrow \mathbb{C}$ depend on a modular parameter $\tau \in \mathbb{H}$ and an elliptic parameter $z \in \mathbb{C}$. They transform under the modular group [19]

$$
\tau \mapsto \tau_{\gamma}=\frac{a \tau+b}{c \tau+d}, \quad z \mapsto z_{\gamma}=\frac{z}{c \tau+d} \quad \text { with } \quad\left(\begin{array}{cc}
a & b \\
a & c
\end{array}\right) \in \operatorname{SL}(2 ; \mathbb{Z})
$$

as

$$
\varphi\left(\tau_{\gamma}, z_{\gamma}\right)=(c \tau+d)^{k} e^{\frac{2 \pi i m c z^{2}}{c \tau+d}} \varphi(\tau, z)
$$

and under quasi periodicity in the elliptic parameter as

$$
\varphi(\tau, z+\lambda \tau+\mu)=e^{-2 \pi i m\left(\lambda^{2} \tau+2 \lambda z\right)} \varphi(\tau, z), \quad \forall \quad \lambda, \mu \in \mathbb{Z} .
$$

Here $k \in \mathbb{Z}$ is called the weight and $m \in \mathbb{Z}_{>0}$ is called the index of the Jacobi form. 


\begin{tabular}{|c|c|c|c|c|c|c|}
\hline & $Q=E_{4}$ & $R=E_{6}$ & $A=\varphi_{-2,1}$ & $B=\varphi_{0,1}$ & $\varphi_{d_{B}}$ & $Z_{d_{B}}(\tau, z)$ \\
\hline weight $\mathrm{k}$ : & 4 & 6 & -2 & 0 & $16 d_{B}$ & 0 \\
\hline index $\mathrm{m}$ : & 0 & 0 & 1 & 1 & $\frac{1}{3} d_{B}\left(d_{B}-1\right)\left(d_{B}+4\right)$ & $\frac{d_{B}\left(d_{B}-3\right)}{2}$ \\
\hline
\end{tabular}

Table 1. $E_{4}, E_{6}, \varphi_{0,1}, \varphi_{-2,1}$ are generators of the ring of weak Jacobi forms with even weights, $\varphi_{d_{B}}(\tau, z)$ captures the all genus amplitudes for the $X_{18}(1,1,1,6,9)$ CY 3 -fold and $Z_{d_{B}}(\tau, z)$ is the ratio of weak Jacobi forms.

The Jacobi forms have a Fourier expansion

$$
\varphi(\tau, z)=\sum_{n, r} c(n, r) q^{n} y^{r}, \quad \text { where } q=e^{2 \pi i \tau}, \quad y=e^{2 \pi i z}
$$

Because of the translation symmetry one has $c(n, r)=: C\left(4 n m-r^{2}, r\right)$, which depends on $r$ only modulo $2 m$. For a holomorphic Jacobi form $c(n, r)=0$ unless $4 m n \geq r^{2}$, for cusp forms $c(n, r)=0$ unless $4 m n>r^{2}$, while for weak Jacobi forms one has only the condition $c(n, r)=0$ unless $n \geq 0$.

According to [19], ${ }^{25}$ a weak Jacobi form of given index $m$ and even modular weight $k$ is freely generated over the ring of modular forms of level one, i.e. polynomials in $R=E_{4}(\tau)$, $P=E_{6}(\tau), A=\varphi_{0,1}(\tau, z), B=\varphi_{-2,1}(\tau, z)$ as

$$
J_{k, m}^{w e a k}=\bigoplus_{j=0}^{m} M_{k+2 j}(\mathrm{SL}(2, \mathbb{Z})) \varphi_{-2,1}^{j} \varphi_{0,1}^{m-j} .
$$

We summarize the weights and index of some important forms in the table 1 . The first generators are defined in (3.21). Our conventions for the elliptic theta function and weak Jacobi forms $A$ and $B$ are the following ${ }^{26}$

$$
\begin{aligned}
\theta_{1}(\tau, z) & =z \cdot \eta(\tau)^{3} \exp \left[\sum_{k=1}^{\infty} \frac{B_{2 k}}{2 k(2 k) !}(i z)^{2 k} E_{2 k}(\tau)\right], \\
A=\varphi_{-2,1}(\tau, z) & =-\frac{\theta_{1}(\tau, z)^{2}}{\eta^{6}(\tau)}, \\
B=\varphi_{0,1}(\tau, z) & =4\left[\frac{\theta_{2}(\tau, z)^{2}}{\theta_{2}(0, \tau)^{2}}+\frac{\theta_{3}(\tau, z)^{2}}{\theta_{3}(0, \tau)^{2}}+\frac{\theta_{4}(\tau, z)^{2}}{\theta_{4}(0, \tau)^{2}}\right] .
\end{aligned}
$$

The weak Jacobi form of index $\varphi_{-2,1}$ has simple product form using the Jacobi triple product for $\theta_{1}$ and $x=\left(2 \sin \left(\frac{\lambda}{2}\right)\right)^{2}=-\left(y^{\frac{1}{2}}-y^{\frac{1}{2}}\right)^{2}$

$$
A=-x \prod_{n=1}^{\infty} \frac{\left(1-y q^{n}\right)^{2}\left(1-y^{-1} q^{n}\right)^{2}}{\left(1-q^{n}\right)^{4}} .
$$

\footnotetext{
${ }^{25} \mathrm{~A}$ review of the theory can be found in [15]. We try to follow the notation used there.

${ }^{26}$ Our conventions for the $\theta$ functions associated to the spin structure on the torus are

$$
\Theta\left[\begin{array}{l}
a \\
b
\end{array}\right](\tau, z)=\sum_{n \in \mathbb{Z}} e^{\pi i(n+a)^{2} \tau+2 \pi i z(n+a)+2 \pi i b}
$$
}

and the Jacobi theta functions $\theta_{1}=i \Theta\left[\begin{array}{c}\frac{1}{2} \\ \frac{1}{2}\end{array}\right], \theta_{2}=\Theta\left[\begin{array}{c}\frac{1}{2} \\ 0\end{array}\right], \theta_{3}=\Theta\left[\begin{array}{l}0 \\ 0\end{array}\right]$ and $\theta_{4}=\Theta\left[\begin{array}{c}0 \\ \frac{1}{2}\end{array}\right]$. 
and the weight zero index one form is one half of the elliptic genus of the $K 3$

$$
\chi(K 3 ; q, y)=2 \varphi_{0,1}(\tau, z)=\left(2 y+20+\frac{2}{y}\right)+\left(\frac{20}{y^{2}}-\frac{128}{y}+216-128 y+20 y^{2}\right) q+\mathcal{O}\left(q^{2}\right)
$$

\subsection{Weak Jacobi Forms and holomorphic anomaly equation}

There is a ring isomorphism between the ring of quasi modular forms and the ring of almost holomorphic forms [48], which are crucial in the solution of the holomorphic anomaly equations in rank one Seiberg Witten theories [40] and local Calabi-Yau spaces [1].

In this section we will show by a related argument that the master holomorphic anomaly equation (4.10) for fibre modularity, which reads for the main example $X_{18}(1,1,1,6,9)$

$$
\left(\partial_{\hat{E}_{2}}+\frac{d_{B}\left(d_{B}-3\right)}{24} \lambda^{2}\right) Z_{d_{B}}(\tau, z)=0
$$

is solved by a weak Jacobi Form of index $m=\frac{d_{B}\left(d_{B}-3\right)}{2}$.

Because of (4.15) and (3.24), given a weak Jacobi form $\varphi_{k, m}(\tau, z)$ one can always define an honest modular form of weight $k$ as follows

$$
\tilde{\varphi}_{k}(\tau, z)=e^{\frac{\pi^{2}}{3} m z^{2} E_{2}(\tau)} \varphi_{k, m}(\tau, z) .
$$

It follows that the weak Jacobi forms $\varphi_{k, m}(\tau, z)$ have a Taylor expansion in $z$ with coefficients that are quasi-modular forms as $[15,19]$

$$
\begin{aligned}
\varphi_{k, m}= & \xi_{0}(\tau)+\left(\frac{\xi_{1}(\tau)}{2}+\frac{m \xi_{0}^{\prime}(\tau)}{k}\right)(2 \pi i z)^{2} \\
& +\left(\frac{\xi_{2}(\tau)}{24}+\frac{m \xi_{1}^{\prime}(\tau)}{2(k+2)}+\frac{m^{2} \xi_{0}^{\prime \prime}(\tau)}{2 k(k+1)}\right)(2 \pi i z)^{4}+\mathcal{O}\left(z^{6}\right) .
\end{aligned}
$$

Here the ${ }^{\prime}=d_{\tau}=\frac{\mathrm{d}}{2 \pi i \mathrm{~d} \tau}$ and $\xi_{\nu} \in \tilde{\mathcal{M}}_{k+2 \nu}\left(\Gamma_{0}\right)$, i.e. the coefficients of can be expressed as polynomials of Eisenstein series $E_{2}(\tau), E_{4}(\tau)$ and $E_{6}(\tau)$. Moreover from (4.24) one has

$$
\left(\partial_{E_{2}}+\frac{m \lambda^{2}}{12}\right) \varphi_{k, m}(\tau, z)=0 \text {. }
$$

Prop. 1 of $[48]^{27}$ implies the claim (4.23). Moreover by (3.25) we can write this as holomorphic anomaly equation

$$
\left(2 \pi i \operatorname{Im}^{2}(\tau) \bar{\partial}_{\bar{\tau}}-\frac{m \lambda^{2}}{4}\right) \hat{\varphi}_{k, m}(\tau, z)=0 .
$$

We note as examples of (4.25) the first coefficients in the expansion of $\phi_{-2,1}(\tau, z)$ and $\phi_{0,1}(\tau, z)$ are

$$
\begin{gathered}
\varphi_{-2,1}(\tau, z)=-z^{2}+\frac{E_{2} z^{4}}{12}+\frac{-5 E_{2}^{2}+E_{4}}{1440} z^{6}+\frac{35 E_{2}^{3}-21 E_{2} E_{4}+4 E_{6}}{362880} z^{8}+\mathcal{O}\left(z^{10}\right), \\
\varphi_{0,1}(\tau, z)=12-E_{2} z^{2}+\frac{E_{2}^{2}+E_{4}}{24} z^{4}+\frac{-5 E_{2}^{3}-15 E_{2} E_{4}+8 E_{6}}{4320} z^{6}+\mathcal{O}\left(z^{8}\right) .
\end{gathered}
$$

\footnotetext{
${ }^{27}$ See also http://people.mpim-bonn.mpg.de/zagier/.
} 
Therefore $\phi_{-2,1}(\tau, z)$ and $\phi_{0,1}(\tau, z)$ can be thought of as quasi-modular forms and we can see that they satisfy the modular anomaly equation

$$
\partial_{E_{2}} \varphi_{-2,1}(\tau, z)=-\frac{z^{2}}{12} \varphi_{-2,1}(\tau, z), \quad \partial_{E_{2}} \varphi_{0,1}(\tau, z)=-\frac{z^{2}}{12} \varphi_{0,1}(\tau, z)
$$

Let us finish the section with a comparison of (4.23), (4.27) with Witten's wave equation for the the topological string partition function that reads [73]

$$
\left(\frac{\partial}{\partial\left(t^{\prime}\right)^{\bar{a}}}+\frac{i}{2} \lambda^{2} C_{\bar{a} \bar{b} \bar{c}} g^{b \bar{b}} g^{c \bar{c}} \frac{D}{D t^{b}} \frac{D}{D t^{c}}\right) Z\left(\lambda, \tau, t_{B}\right)=0 .
$$

If we apply this equation to $Z$ defined in (4.9) with $\left(t^{\prime}\right)^{\bar{a}}=\bar{\tau}$ and $Q^{\beta}=e^{2 \pi i d_{B} t_{B}}$, we get in the large base because of the special from of the intersection matrix of elliptically fibered Calabi-Yau 3 folds only derivatives in the base direction $t_{B}$ for $t^{b}$ and $t^{c}$. Identifying $\lambda$ with $z$ we see already that the index will grow quadratically in $d_{B}$. A more detailed analysis as in [54] shows also the shift by $K_{B}$ so that the large base limit of (4.30) becomes equivalent to all equations (4.10).

\subsection{Exact formulae for base degree zero}

For base degree $\underline{d}_{B}=\underline{0}$, since the BPS numbers $n_{d_{E}, \underline{\underline{0}}}^{(g)}=0$ for genus $g \geq 2$, we get from (5.1) the formula

$$
P_{0}^{(g)}=-\chi \frac{B_{2 g}}{2 g(2 g-2) !}\left(\frac{B_{2 g-2}}{2(2 g-2)}-\sum_{d=1}^{\infty} \operatorname{Li}_{3-2 g}\left(q^{d}\right)\right), \quad g \geq 2,
$$

where $\chi$ is the Euler number. This formula holds not just for the cases of elliptic fibrations with only $I_{1}$ Kodaira fibers [54], whose toric description is reviewed in section 2, but also for the case discussed in [28] with higher Kodaira fibre and non-abelian gauge symmetries.

We recognize in the multi-cover formula (4.31) the well-known formula for Eisenstein series

$$
P_{0}^{(g)}=-\chi \frac{B_{2 g} B_{2 g-2}}{8 g(g-1)(2 g-2) !} E_{2 g-2}(q), \quad g \geq 2 .
$$

So we see for $\chi=-540$ that $P_{0}^{(2)}=-\frac{3}{32} E_{2}$, and in general $P_{0}^{(g)}$ is a $\operatorname{SL}(2, \mathbb{Z})$ modular form of weight $2 g-2$ for $g>2$.

For the $d_{B}=0$ genus one amplitude for our case, we can compute the base degree zero amplitude $P_{0}^{(1)}$ from the formula (3.17) and modularity formulas proven in [39]. Taking $z_{2} \rightarrow 0$ of theses, we compute the determinant of Kähler metric as $\operatorname{det}(G) \sim$ $\left(\partial_{z_{1}} t_{E}\right)\left(\partial_{z_{2}} t_{B}\right) \sim \frac{E_{4}\left(q_{E}\right)^{\frac{5}{2}}}{\eta\left(q_{E}\right)^{24}} \frac{1}{z_{2}}$, with $\partial_{z_{1}} t_{E}=\frac{E_{4}\left(q_{E}\right)^{\frac{5}{2}}}{\eta\left(q_{E}\right)^{24}}+\mathcal{O}\left(z_{2}\right)$. We can hence write the genus one amplitude in the $z_{2} \rightarrow 0$ limit as

$$
\mathcal{F}^{(1)}=-\frac{25}{4} \log \left(E_{4}\right)-\frac{1}{2} \log \left(\frac{E_{4}\left(q_{E}\right)^{\frac{5}{2}}}{\eta\left(q_{E}\right)^{24}}\right)-\frac{19}{4} \log \left(z_{1}\right)-\frac{3}{2} \log \left(z_{2}\right)-\frac{1}{12} \log \left(\Delta_{1} \Delta_{2}\right)+\mathcal{O}\left(z_{2}\right) .
$$


A careful calculation show that

$$
P_{0}^{(1)}=-48 \log \left(\eta\left(q_{E}\right)\right)-\frac{3}{2} \log \left(q_{B} q_{E}^{\frac{3}{2}}\right) .
$$

For the $d_{B}=0$ genus the zero amplitude $P_{0}^{(0)}$ has formally weight -2 . We therefore consider the three point coupling which is the well defined observable after fixing the conformal Killing symmetries on the world-sheet. The classical contribution is a cubic polynomial of the flat coordinates (2.16), which encode the classical triple intersection numbers. Similar to genus one formula (4.34), we should shift the base flat coordinate according to (4.1) and find

$$
\partial_{t_{E}}^{3} P_{0}^{(0)}=\frac{9}{4} E_{4}\left(q_{E}\right)
$$

which confirms the effective modular weight of -2 since each derivative increases modular weight by 2 . The modularity at genus zero in this limit is a consequence of (3.13) and equation (4.35) has been observed in [12].

\subsection{Exact formulae for higher base degrees}

Our main claim is that the all genus partition function for the topological string elliptic fibration over $\mathbb{P}^{2}$ realized e.g. as degree 18 hypersurface in the weighted projective space is given by

$$
Z_{d_{B}}(\tau, z)=\frac{\varphi_{d_{B}}(\tau, z)}{\eta(\tau)^{36 d_{B}} \prod_{s=1}^{d_{B}} \varphi_{-2,1}(\tau, s z)},
$$

where $\varphi_{d_{B}}$ is an weak Jacobi form of weight $k=16 d_{B}$ and index $m=\frac{d_{B}}{3}\left(d_{B}-1\right)\left(d_{B}+4\right)$. Scaling $z$ in $\varphi_{k, m}(\tau, z) \in J_{k, m}$ by $s$ corresponds to a Hecke-like operator $U_{s}: J_{k, m}^{*} \rightarrow J_{k, s^{2} m}^{*}$. The weights and indices of the relevant weak Jacobi forms are summarized in table 1. Since

$$
\operatorname{dim} M_{k}(\mathrm{SL}(2, \mathbb{Z}))=\left\{\begin{array}{l}
{[k / 12]+1 \text { if } k \neq 2 \bmod 12} \\
{[k / 12] \quad \text { if } k=2 \bmod 12}
\end{array}\right\}
$$

the number of coefficients in $\varphi_{d_{B}}^{t s}$ grows like $\frac{d_{B}^{6}}{108}$ for large $d_{B}$. The first few numbers for $d_{b}=1,2, \ldots$ are $2,17,84,278,737,1692,3501, \ldots$

For the case of $d_{B}=1$ the two coefficients are fixed by two genus 0 BPS numbers

$$
\varphi_{1}=-\frac{Q\left(31 Q^{3}+113 R^{2}\right)}{48} .
$$

This determines for base degree 1 all genus BPS invariants by (4.36), (4.9), (4.5) and the multi-covering formula (5.1). Up to $g=6$ and $d_{E}=6$ we list them in the table (2). We notice that they match all the numbers that have been obtained to genus 8 using the direct integration of the holomorphic anomaly condition, the involution symmetry and the conifold gap condition. A detailed discussion on the perfect matching with the accessible enumerative invariants can be found in section 5.2.1 


\begin{tabular}{|c|c|c|c|c|c|c|c|}
\hline$g \backslash d_{E}$ & $d_{E}=0$ & 1 & 2 & 3 & 4 & 5 & 6 \\
\hline$g=0$ & 3 & -1080 & 143370 & 204071184 & 21772947555 & 1076518252152 & 33381348217290 \\
\hline 1 & 0 & -6 & 2142 & -280284 & -408993990 & -44771454090 & -2285308753398 \\
\hline 2 & 0 & 0 & 9 & -3192 & 412965 & 614459160 & 68590330119 \\
\hline 3 & 0 & 0 & 0 & -12 & 4230 & -541440 & -820457286 \\
\hline 4 & 0 & 0 & 0 & 0 & 15 & -5256 & 665745 \\
\hline 5 & 0 & 0 & 0 & 0 & 0 & -18 & 6270 \\
\hline 6 & 0 & 0 & 0 & 0 & 0 & 0 & 21 \\
\hline
\end{tabular}

Table 2. Some BPS invariants $n_{\left(d_{E}, 1\right)}^{g}$ for base degree $d_{B}=1$ and $g, d_{E} \leq 6$ as determined by (4.38) for all $g, d_{E}$.

Note that (4.36) implies Hilbert-scheme like infinite product formulae for the generating functions. E.g. for $d_{B}=1$ one gets

$$
Z_{1}(\tau, z)=\left[\frac{1}{2 i \sin \left(\frac{z}{2}\right) \eta(\tau)^{18}} \prod_{n=1}^{\infty} \frac{\left(1-q^{n}\right)^{2}}{\left(1-e^{i z} q^{n}\right)\left(1-e^{-i z} q^{n}\right)}\right]^{2} \varphi_{1}
$$

where $B_{2 k}$ are the Bernoulli numbers. This infinite product formula alone eliminates the subspace $V_{ \pm}^{\left(1,\left[\frac{g-1}{3}\right], 1\right)}$ in the holomorphic ambiguity (E.8) and allows already to topological string amplitudes up to $g \leq 18$ for this model.

For the $d_{B}=2$ case three of 17 coefficients can be already fixed by demanding that there is no pole $z^{-4}=\lambda^{-4}$ in $P_{2}(\tau, z)$. Note that this pole has to be canceled by the $\left(Z_{1}\right)^{2}$ contribution in $P_{2}(\tau, z)$. This explains the first term in (4.42). The vanishing bound which we will demonstrate in section 5.2.1 is a Castelnuovo-like criterium, namely that

$$
n_{d_{E}, 2}^{g}=0, \quad \text { for } \quad d_{E} \geq 5, g \geq 2 d_{E}-3
$$

fixes eleven other constants and implies all vanishing on the right from the edge. The actual nonzero values of the BPS numbers on the generic part of the edge for $d_{E} \geq 5$

$$
n_{d_{E}, 2}^{2 d_{E}-4}=12-6 d_{E}
$$

are then calculated geometrically and yield no further constraints. In order to fix the remaining three constants one needs the information of any three nonzero numbers in the same row or the same column way from the numbers (4.41). Notice that three numbers $n_{d_{E}, 2}^{2 d_{E}-5}, d_{E}=5+i, i=0,1,2$ do yield only one constraint. Random patterns of nonzero numbers away from (4.41) will lead in general to independent equations. The result e.g. fixed from three genus zero numbers gives

$$
\begin{aligned}
\varphi_{2}= & \frac{B^{4} Q^{2}\left(31 Q^{3}+113 R^{2}\right)^{2}}{23887872}+\frac{1}{1146617856}\left[2507892 B^{3} A Q^{7} R+9070872 B^{3} A Q^{4} R^{3}\right. \\
& +2355828 B^{3} A Q R^{5}+36469 B^{2} A^{2} Q^{9}+764613 B^{2} A^{2} Q^{6} R^{2}-823017 B^{2} A^{2} Q^{3} R^{4} \\
& +21935 B^{2} A^{2} R^{6}-9004644 B A^{3} Q^{8} R-30250296 B A^{3} Q^{5} R^{3}-6530148 B A^{3} Q^{2} R^{5} \\
& \left.+31 A^{4} Q^{10}+5986623 A^{4} Q^{7} R^{2}+19960101 A^{4} Q^{4} R^{4}+4908413 A^{4} Q R^{6}\right],
\end{aligned}
$$

which predicts the BPS numbers in all genus and fibre classes for $d_{B}=2$. 


\begin{tabular}{|c|c|c|c|c|c|c|c|}
\hline$g \backslash d_{E}$ & $d_{E}=0$ & 1 & 2 & 3 & 4 & 5 & 6 \\
\hline$g=0$ & -6 & 2700 & -574560 & 74810520 & -49933059660 & 7772494870800 & 31128163315047072 \\
\hline 1 & 0 & 15 & -8574 & 2126358 & 521856996 & 1122213103092 & 879831736511916 \\
\hline 2 & 0 & 0 & -36 & 20826 & -5904756 & -47646003780 & -80065270602672 \\
\hline 3 & 0 & 0 & 0 & 66 & -45729 & 627574428 & 3776946955338 \\
\hline 4 & 0 & 0 & 0 & 0 & -132 & -453960 & -95306132778 \\
\hline 5 & 0 & 0 & 0 & 0 & 0 & -5031 & 1028427996 \\
\hline 6 & 0 & 0 & 0 & 0 & 0 & -18 & -771642 \\
\hline 7 & 0 & 0 & 0 & 0 & 0 & 0 & -7224 \\
\hline 8 & 0 & 0 & 0 & 0 & 0 & 0 & -24 \\
\hline
\end{tabular}

Table 3. Some BPS invariants for $n_{\left(d_{E}, 2\right)}^{g}$.

For $d_{B}=3$ the vanishing, given by

$$
n_{d_{E}, 3}^{g}=0, \quad \text { for } \quad d_{E} \geq 8, g \geq 3 d_{E}-10
$$

fixes 74 other constants. These conditions are not independent from the conditions that eliminate the $z^{-6}$ and $z^{-4}$ poles and implies all vanishing on the right from the edge. If we impose the conditions successively we get $\{25,47,58,63,67,70,71,72,73, \overline{74}\}$ conditions for $d_{E}=8,9, \ldots$. The genus zero and one invariants yield 5 and 5 conditions fixing all coefficients of $\varphi_{3}$.

As we will argue in section 5.2.1 the vanishing conditions for general $d_{B}$ is

$$
n_{d_{E}, d_{B}}^{g}=0, \quad \text { for } \quad d_{E} \geq 3 d_{B}-1, g \geq d_{B} d_{E}-\frac{1}{2}\left(3 d_{B}^{2}-d_{B}-4\right) .
$$

Together with the data up to genus eight tabulated in appendix A, this allows to fix $\varphi_{4}$ with 20 and $\varphi_{5}$ with 2 nontrivial checks respectively. ${ }^{28}$ The tables of base degree $d_{B}=4$ and $d_{B}=5$ to genus 27 and 41 respectively can be found likewise in the appendix. We appended the expression for $\varphi_{d}, d=1, \ldots, 5$ in a for algebraic programs readable form to this TeX file.

One can make further checks on the results based on considerations in section (5.2.1). For examples we see that for each $d_{B}$ there is a critical value

$$
\left(g^{c}\left(d_{B}\right)=\frac{1}{2}\left(3 d_{B}^{2}-d_{B}+2\right), d_{E}^{c}\left(d_{B}\right)=3 d_{B}-1\right)
$$

in the $\left(g, d_{E}\right)$ plane so that the BPS states on the line in the $\left(g, d_{E}\right)$ takes the value

$$
n_{d_{E}, d_{B}}^{d_{B} d_{E}-g_{c}\left(d_{B}\right)+2}=(-1)^{\frac{2 d_{E} d_{B}+d_{B}\left(d_{B}-1\right)}{2}} 3\left(d_{B} d_{E}-\left(3 d_{B}^{2}+d_{B}-6\right) / 2\right) \text { for } d_{E} \geq 3 d_{B}-1,
$$

which can be confirmed as well as many other predictions by direct curve counting in section 5.2.1.

These formulas (4.38), (4.42) are valid for all genera, so we can consider their implications for Gopakumar-Vafa (GV) invariants at large genus. We use the Mathematica

\footnotetext{
${ }^{28}$ We have checked the results now at genus nine, which gives 18 new independent checks for $\varphi_{4}$ and 20 for $\varphi_{5}$. The data are available on request.
} 


\begin{tabular}{|c|c|c|c|c|c|c|c|c|c|c|}
\hline$g \backslash d_{E}$ & 0 & 1 & 2 & 3 & 4 & 5 & 6 & 7 & 8 & 9 \\
\hline 0 & 27 & -17280 & 5051970 & $-91(5) 00$ & $22(8) 00$ & $-42(10) 68$ & $40(12) 60$ & $-16(16) 20$ & $55(17) 80$ & $12(22) 00$ \\
\hline 1 & -10 & 4764 & -1079298 & $15(5) 86$ & $-16(7) 80$ & $-33(9) 68$ & $12(12) 88$ & $-55(14) 44$ & $10(18) 24$ & $37(21) 76$ \\
\hline 2 & 0 & 27 & -16884 & 4768830 & $-81(5) 36$ & $28(8) 63$ & $67(10) 51$ & $45(13) 02$ & $36(16) 60$ & $54(19) 78$ \\
\hline 3 & 0 & 0 & -72 & 48036 & $-14(4) 90$ & $29(5) 64$ & $-79(9) 12$ & $-63(12) 64$ & $-94(15) 98$ & $-11(19) 86$ \\
\hline 4 & 0 & 0 & 0 & 154 & -110574 & $38(4) 41$ & $21(8) 04$ & $50(11) 76$ & $12(15) 04$ & $20(18) 021$ \\
\hline 5 & 0 & 0 & 0 & 0 & -306 & 247014 & $-25(6) 52$ & $-21(10) 36$ & $-11(14) 06$ & $-30(17) 40$ \\
\hline 6 & 0 & 0 & 0 & 0 & 0 & 612 & 1401468 & $50(8) 62$ & $74(12) 04$ & $34(16) 43$ \\
\hline 7 & 0 & 0 & 0 & 0 & 0 & 0 & 17386 & $-49(6) 70$ & $-35(11) 70$ & $-32(15) 78$ \\
\hline 8 & 0 & 0 & 0 & 0 & 0 & 0 & 63 & 3396663 & $11(10) 79$ & $23(14) 91$ \\
\hline 9 & 0 & 0 & 0 & 0 & 0 & 0 & 0 & 32418 & $-21(8) 14$ & $-13(13) 22$ \\
\hline 10 & 0 & 0 & 0 & 0 & 0 & 0 & 0 & 108 & $18(6) 07$ & $57(11) 85$ \\
\hline 11 & 0 & 0 & 0 & 0 & 0 & 0 & 0 & 0 & -1151442 & $-16(10) 36$ \\
\hline 12 & 0 & 0 & 0 & 0 & 0 & 0 & 0 & 0 & -10917 & $29(8) 80$ \\
\hline 13 & 0 & 0 & 0 & 0 & 0 & 0 & 0 & 0 & -36 & $-24(6) 86$ \\
\hline 14 & 0 & 0 & 0 & 0 & 0 & 0 & 0 & 0 & 0 & 1458792 \\
\hline 15 & 0 & 0 & 0 & 0 & 0 & 0 & 0 & 0 & 0 & 13770 \\
\hline 16 & 0 & 0 & 0 & 0 & 0 & 0 & 0 & 0 & 0 & 45 \\
\hline
\end{tabular}

Table 4. Some BPS invariants for $n_{\left(d_{E}, 3\right)}^{g}$. To save space we only give the first and the last two significant digits and the number of omitted digits in brackets.

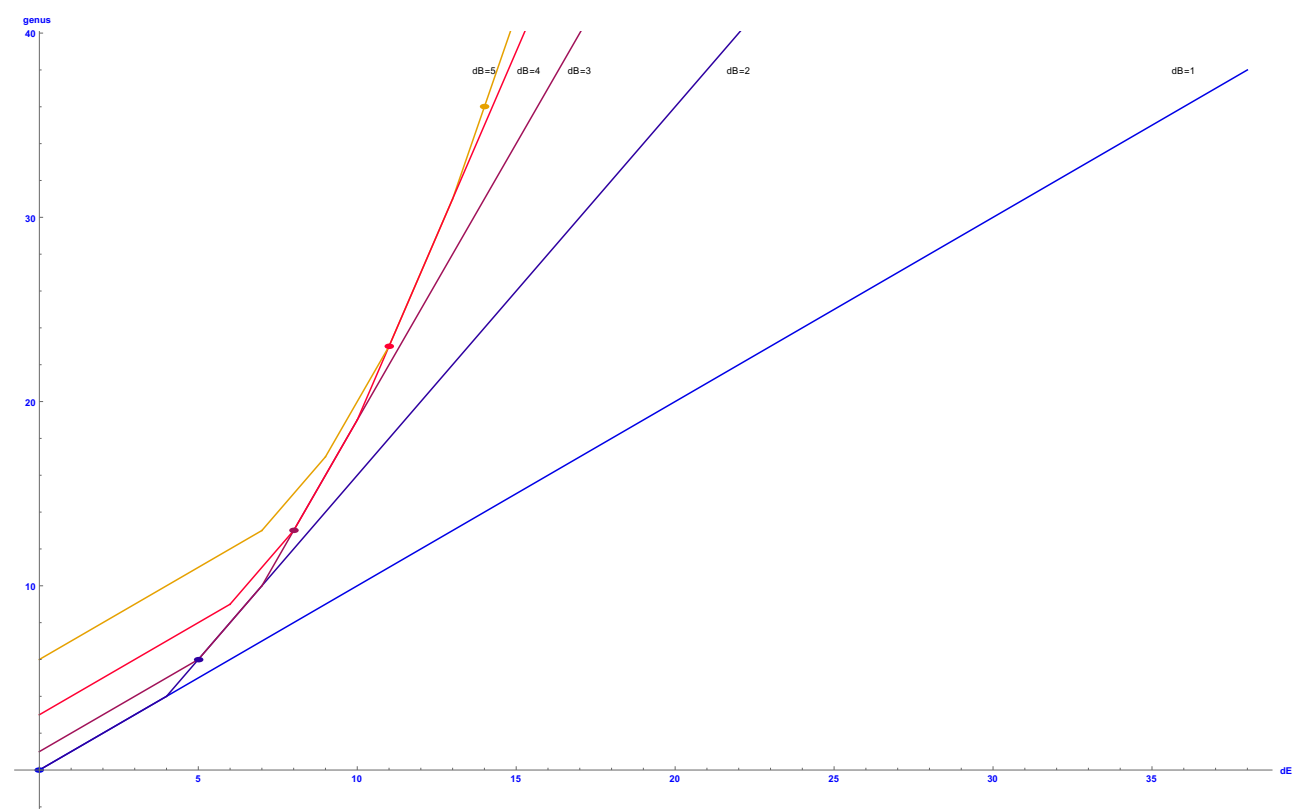

Figure 1. The figure shows the boundary of non-vanishing curves for the values of $d_{B}=1,2,3,4,5$. The dot on each $d_{B}$ line at $\left(g^{c}\left(d_{B}\right)=\frac{1}{2}\left(3 d_{B}^{2}-d_{B}+2\right), d_{E}^{c}\left(d_{B}\right)=3 d_{B}-1\right)$ indicates the value at which the boundary slope becomes generic and the numbers of BPS states on the line are given by (4.46). 
program to compute the GV invariants to genus 8, and the results are listed in the tables in the appendix. A well-known feature of the GV invariants from algebraic geometric arguments [50] is that, for a given 2nd-homology class, in our case a pair of fiber and base degrees $\left(d_{E}, d_{B}\right)$, the GV invariant $n_{\left(d_{E}, d_{B}\right)}^{g}$ vanishes for sufficiently large genus $g$. The largest genus with non-vanishing GV invariant is called the top genus for the given degrees $\left(d_{E}, d_{B}\right)$. For example, from the GV tables in the appendix A we can see that the top genus for the degrees $\left(d_{E}, 1\right)$ is always $d_{E}$, while the top genus for $\left(d_{E}, 2\right)$ is $d_{E}$ for the cases of $d_{E} \leq 4$, and is 6,8 for $d_{E}=5,6$ respectively. The situation is summarized in figure 1 and the bounds are verified from algebraic geometry in section 5.2.

We consider whether such vanishing conditions help to fix the unknown coefficients in the ansatz of the weak Jacobi form. It turns out for the first case of $d_{B}=1$, a general ansatz (4.36) ensures the vanishings of $n_{\left(d_{E}, 1\right)}^{g}=0$ for $g>d_{E}$, therefore this does not help to fix the two coefficients in the numerator in the formula (4.38). To understand this structure, let us analyze the formula for $\phi_{-2,1}(\tau, z)$ in (4.20), which can be also written in the infinite product form. For comparing with GV invariants, we define the variable $x=\left(2 \sin \left(\frac{z}{2}\right)\right)^{2}$, then we find

$$
\phi_{-2,1}(\tau, z)=-x \prod_{n=1}^{\infty} \frac{\left(1+x q^{n}-2 q^{n}+q^{2 n}\right)^{2}}{\left(1-q^{n}\right)^{4}} .
$$

So we see that excluding the total factor of $x$, in the power series expansion of the infinite product, the power of $q$ is always no less than that of $x$. Using the series expansion $(1+x)^{-2}=\sum_{n=0}^{\infty}(-1)^{n}(n+1) x^{n}$, we find the inverse can be written as

$$
\frac{1}{\phi_{-2,1}(\tau, z)}=-\frac{1}{x} \sum_{n=0}^{\infty}(-1)^{n}(n+1) q^{n}\left[x^{n}+f_{n-1}(x)\right],
$$

where $f_{n-1}(x)$ is a polynomial of $x$ of degree $n-1$. Topological string free energy in the $d_{B}=1$ case has no multi-cover contributions from lower degrees, and according to the general ansatz (4.36) is simply $\phi_{-2,1}(\tau, z)^{-1}$ multiplying a modular form of weight 16 and the factor of $\left(\frac{q}{\left.\eta(q)^{24}\right)}\right)^{\frac{3}{2}}$, which only increase the $q$ power in the series expansion. Here the factor $q^{\frac{3}{2}}$ comes from the shift of base Kahler parameter such that the partition function has a nice modular form. From the well-known GV expansion of the topological free energy, the coefficient of $q^{d_{E}} x^{g-1}$ is exactly the GV invariant $n_{\left(d_{E}, 1\right)}^{g}$. Therefore we see from the expansion (4.48) that indeed the GV invariant $n_{\left(d_{E}, 1\right)}^{g}$ vanishes for $g>d_{E}$. Furthermore, using the formula (4.38) we can easily calculate the top genus numbers $n_{(g, 1)}^{g}=3(-1)^{g}(g+1)$. This agrees with the numbers in the tables of GV invariants and can be also derived from algebraic geometric arguments in section 5.2.1.

Now we consider the general cases of higher base degrees. Similar to the case of $\phi_{-2,1}(\tau, z)$ in (4.47), we can also expand $\phi_{0,1}(\tau, z)$ as power series of $x, q$

$$
\phi_{0,1}(\tau, z)=-x\left(1-10 x q+x^{2} q^{2}\right)+\sum_{n=0}^{\infty} q^{n} f_{n}(x),
$$


where $f_{n}(x)$ symbolizes a degree $n$ polynomial of $x$. Overall the difference of the powers of $x$ and $q$ in both the series $\phi_{-2,1}(\tau, z)$ and $\phi_{0,1}(\tau, z)$ is always no larger than 1 . So we can consider the sub-family of ansatz with only $\phi_{-2,1}(\tau, z)$ in the denominator, namely

$$
\sum_{k=-1}^{\frac{d_{B}\left(d_{B}-3\right)}{2}} \frac{f_{18 d_{B}+2 k}\left(E_{4}, E_{6}\right)}{\eta(\tau)^{36 d_{B}}} \phi_{-2,1}(\tau, z)^{k} \phi_{0,1}(\tau, z)^{\frac{d_{B}\left(d_{B}-3\right)}{2}-k},
$$

where $f_{18 d_{B}+2 k}\left(E_{4}, E_{6}\right)$ symbolizes a modular form of weight $18 d_{B}+2 k$, which is a polynomials of $E_{4}$ and $E_{6}$ with many unknown coefficients, so that the total modular weight and index are the same as the more general ansatz (4.36) with the product $\prod_{k=1}^{d_{B}} \phi_{-2,1}(\tau, k z)$ in the denominator. From the power series of $\phi_{-2,1}(\tau, z)$ and $\phi_{0,1}(\tau, z)$ in $x, q$ and including the factor of $q^{\frac{3 d_{B}}{2}}$ from the shift of base Kahler parameter, we deduce that the sub-family of ansatz (4.50) contribute only to the GV invariants $n_{\left(d_{E}, d_{B}\right)}^{g}$ with $g \leq d_{E}+\frac{d_{B}\left(d_{B}-3\right)}{2}+1$. On the other hand, from the algebro-geometric arguments in section 5.2.1, the top genus with non-vanishing GV invariants for the case of $d_{E}=0$ is always $\frac{d_{B}\left(d_{B}-3\right)}{2}+1$. For a given $d_{B}$, the top genus as a function of $d_{E}$ is strictly monotonically increasing, i.e. the top genus for $\left(d_{E}+1, d_{B}\right)$ is always strictly bigger than that of $\left(d_{E}, d_{B}\right)$. Therefore we deduce that the top genus with non-vanishing GV invariants for degrees $\left(d_{E}, d_{B}\right)$ is always no less than $d_{E}+\frac{d_{B}\left(d_{B}-3\right)}{2}+1$. Furthermore, when $d_{E}$ is sufficiently large, from the algebraic geometric arguments, the top genus is given by a formula $d_{B} d_{E}+\frac{1}{2}\left(-3 d_{B}^{2}+d_{B}+2\right)$, which is certainly much larger than $d_{E}+\frac{d_{B}\left(d_{B}-3\right)}{2}+1$. We can choose a particular solution from the general ansatz (4.36), so that the GV invariants vanish at sufficiently large genus, then the addition of extra ansatz of the form (4.50) does not affect the conditions. So we conclude that the sub-family of ansatz (4.50) can not be fixed by vanishing GV conditions.

The reverse is also true, namely, any remaining ambiguities which can not be fixed by vanishing GV conditions are necessarily of the form (4.50). To see this, we note that $\left(2 \sin \left(\frac{k z}{2}\right)\right)^{2}$ can be written as a polynomial of $x \equiv\left(2 \sin \left(\frac{z}{2}\right)\right)^{2}$ of degree $k$, more explicitly

$$
\left(2 \sin \left(\frac{k z}{2}\right)\right)^{2}=x f_{k-1}(x)
$$

where $f_{k-1}(x)$ is a degree $k-1$ polynomial, related to the Chebyshev polynomials. For example, for $k=2$, we have $(2 \sin z)^{2}=x(4-x)$. The constant term $f_{k-1}(0)$ in the polynomial is always $k^{2}$ since we have $\frac{\left(2 \sin \left(\frac{k z}{2}\right)\right)^{2}}{x} \sim k^{2}$ in the limit $x \sim z \sim 0$. When we expand $\frac{1}{\phi_{-2,1}(\tau, k z)}$ for $k>1$ as power series of $x, q$, we see that the power of $x$ has no upper bound for a given power of $q$, unlike the case of $k=1$. So if the factor $f_{k-1}(x)$ is not cancelled in the denominator, the ansatz would contribute to the GV invariants $n_{\left(d_{E}, d_{B}\right)}^{g}$ of arbitrarily large genus $g$ for the given degrees $\left(d_{E}, d_{B}\right)$, and we can always fix them with vanishing GV invariants, even without the precise knowledge of the top genus. Furthermore, we require that the power of $x$ in the generating function of the GV invariants is always no less than -1 , since the lowest genus is zero. Those ansatz that contribute to $x^{-n}$ with $n>1$ can be always fixed by this requirement. Overall we have at most one factor $x$ in the denominator for those ansatz that are not affected by these considerations. Thus all ansatz except for those of the form (4.50) can be fixed in this way. 
This is checked by actual calculations. One can easily count that the sub-family of ansatz (4.50) has 2,3,10,26 unknown coefficients for base degrees $d_{B}=1,2,3,4$ respectively. After we impose the conditions that the generating function of GV invariants start from genus zero and vanish at sufficiently large genus for a given fiber degree, these are the indeed the remaining numbers of unfixed coefficients in the more general ansatz (4.36) with the denominator $\prod_{k=1}^{d_{B}} \phi_{-2,1}(\tau, k z)$.

We can also see the scaling of the top genus for a given base degree $d_{B}$ and for large fiber degree $d_{E}$. We can expand more explicitly

$$
\frac{1}{\phi_{-2,1}(\tau, k z)}=-\frac{1}{x f_{k-1}(x)}+\sum_{n=1}^{\infty} q^{n} g_{n-1}\left(x f_{k-1}(x)\right),
$$

where $f_{k-1}(x)$ is the polynomial in (4.51) and $g_{n-1}$ is a degree $n-1$ polynomial. There are also multi-cover contributions from lower degrees. For example, we can consider $k$-cover contributions from the base degree one formula. The denominator can be expanded as

$$
\frac{1}{\phi_{-2,1}(k \tau, k z)}=-\frac{1}{x f_{k-1}(x)}+\sum_{n=1}^{\infty} q^{k n} g_{n-1}\left(x f_{k-1}(x)\right),
$$

where the polynomials $f_{k-1}, g_{n-1}$ are the same as in (4.52). We see that the first terms, which would have unbounded powers of $x$ in series expansion, are the same. At the end, the polynomial $f_{k-1}(x)$ must be cancelled in the denominator, so that the resulting GV invariants have a top genus. In this way we see that the minimal requirements for the denominator of the ansatz at base degree $d_{B}$ is indeed $\prod_{k=1}^{d_{B}} \phi_{-2,1}(\tau, k z)$, otherwise there will be non-vanishing GV invariants at arbitrarily large genus for fixed degrees from the multi-cover contributions. Since the factor $f_{k-1}(x)$ in the denominator of the first term in (4.52) is eventually canceled, this term contributes a finite polynomial of $x$ in the generating function of GV invariants and does not affect the leading scaling behavior of the top genus for large fiber degree. At the base degree $d_{B}$, the leading contribution to the top genus comes from the second term in (4.52) for $k=d_{B}$ with large $n$, and scales like $g_{\text {top }} \sim d_{B} d_{E}$. However it is more tricky to determine the exact formula in this way for the cases of $d_{B}>1$. We find that the exact top genus numbers are not universal for the general ansatz (4.36), and the top genus formula $d_{B} d_{E}+\frac{1}{2}\left(-3 d_{B}^{2}+d_{B}+2\right)$ for large $d_{E}$ derived from algebraic arguments only appears for the the particular solution in topological string theory.

This approach can be combined with the B-model holomorphic anomaly approach to compute higher genus topological string amplitudes. Using the involution symmetry and the boundary conditions at the conifold point, we find that the exact formula at base degree $d_{B}$ can provide sufficient boundary data to fix the B-model formula at genus $9\left(d_{B}+1\right)$, valid for all base and fiber degrees, see the appendixes for more details. On the other hand, in order to fix the exact formula at base degree $d_{B}$, we need to fix the ansatz (4.50) with some non-vanishing GV invariants. In particular, consider the term in (4.50) with $k=\frac{d_{B}\left(d_{B}-3\right)}{2}$ in the sum. Since $\phi_{-2,1}(\tau, z) \sim x$ in the power series expansion of $x, q$, this term contributes only to and can be only fixed by topological string free energy or some nonvanishing GV invariants of genus no less than $\frac{d_{B}\left(d_{B}-3\right)}{2}+1$. The contributions of the other 
terms in (4.50) start from lower genus. On the other hand, the modular form coefficients in the ansatz (4.50) have different modular weights for different $k$, and their contributions can not cancel when we extract the contributions at a given genus by expanding for small $z$. Therefore if we only know the B-model formula at any genus no less than $\frac{d_{B}\left(d_{B}-3\right)}{2}+1$, it is also already sufficient to fix the ansatz (4.50) at base degree $d_{B}$. This is checked by actual low base degrees calculations. Thus, as long as $9\left(d_{B}+1\right) \geq \frac{\left(d_{B}+1\right)\left(d_{B}-2\right)}{2}+1$, we can repeat this procedure to fix the exact formula with increasing base degrees. If no other obstacle arises, we foresee the eventual termination of this recursive process only at $d_{B}=20$. In this way we can in principle determine the exact formula up to base degree $d_{B}=20$ (for all genera and fiber degrees), and the topological string free energy up to genus 189 (for all base and fiber degrees). In practice we provide here the solution of the topological strings up to $d_{B}=5$ for all genera and genus $g=8$ for all degrees.

\section{BPS invariants}

After a technical recapitulating of the the physical definition of the BPS invariants in section 5.1, the main part of the section is devoted to the direct geometrical calculation of the unrefined BPS invariants or Gopakumar-Vafa invariants for the $X_{18}(1,1,1,6,9)$ CalabiYau 3-fold in section 5.2.

\subsection{Physical definition of the BPS invariants}

In this section we recall the definition of the integer BPS invariants from $\mathcal{F}(\lambda, \underline{t})$ or $\mathcal{Z}(\lambda, \underline{t})$. In view of the similarity of the all genus expansions discussed in section 4.6 and the product expansion of (1.4) based on the Borcherds lifting of automorphic forms of $O(2, n, \mathbb{Z})$, see [8] and [59] the formulae (5.5) and (5.11) should give further hints how to characterize $\mathcal{Z}$. We comment on possible refinements in 5.1.2 in analogy to the $E$-string.

\subsubsection{Unrefined BPS invariants}

The unrefined BPS states multiplicities $n_{\beta}^{g} \in \mathbb{Z}$ are defined by their contribution to a Schwinger-Loop integral in $5 d N=2$ supergravity theory $[24]^{29}$

$$
\mathcal{F}(\lambda, \underline{t})=\operatorname{class}(\underline{t})+\sum_{\beta \in H_{2}\left(M_{3}, \mathbb{Z}\right)} \sum_{g=0}^{\infty} \sum_{m=1}^{\infty} \frac{n_{\beta}^{g}}{m}\left(2 \sin \left(\frac{m \lambda}{2}\right)\right)^{2 g-2} q^{\beta m} .
$$

Note that $q^{\beta}=\exp \left(2 \pi i \sum_{\alpha=1}^{b_{2}\left(M_{3}\right)} t^{\alpha} \beta_{\alpha}\right)$ and $y^{\frac{m}{2}}-y^{-\frac{m}{2}}=2 i \sin \left(\frac{m \lambda}{2}\right)$. One can interpret $\beta$ as BPS charge and $\sum_{\alpha} t^{\alpha} \beta_{\alpha}$ as the central charge mass term. In the 5 -field theory the fugacity $\lambda$ traces the left spin of the BPS states.

The $n_{\beta}^{g} \in \mathbb{Z}$ may be calculated by identifying the above expression to the perturbative topological string expansion on $M$

$$
\mathcal{F}(\lambda, \underline{t})=\operatorname{class}(\underline{t})+\sum_{g=0}^{\infty} \lambda^{2 g-2} \mathcal{F}^{g}(\underline{t})=\sum_{g=0}^{\infty} \sum_{\beta \in H_{2}(M, \mathbb{Z})} \lambda^{2 g-2} r_{\beta}^{g} q^{\beta},
$$

\footnotetext{
${ }^{29}$ Caligraphic letters should refer to quantities in the holomorphic limit.
} 
where $\lambda$ is now the topological string coupling constant. The Gromov-Witten invariants $r_{\beta}^{g} \in \mathbb{Q}$ can be mathematically defined and in suitable cases calculated using localization. In this article we use mirror symmetry, modularity, the holomorphic anomaly equation and direct curve counting to obtain the $F^{(g)}(\underline{t})$. With

$$
\sum_{m=1}^{\infty} \frac{1}{m} \frac{q^{m}}{\left(2 \sin \left(\frac{m \lambda}{2}\right)\right)^{2}}=\sum_{g=0}^{\infty} \lambda^{2 g-2}(-1)^{g+1} \frac{B_{2 g}}{2 g(2 g-2) !} \operatorname{Li}_{3-2 g}(q)
$$

where $\operatorname{Li}_{n}(x)=\sum_{k=1}^{\infty} \frac{x^{k}}{k^{m}}$ and the Bernoulli numbers are defined in (3.23). Using further $\zeta(-n)=-\frac{B_{n+1}}{n+1}$ for $n \in \mathbb{N}_{+}$we get

$$
F_{g}(\underline{t})=(-1)^{g+1} n_{0}^{0} \frac{\left|B_{2 g} B_{2 g-2}\right|}{2 g(2 g-2)(2 g-2) !}+\mathcal{O}(\underline{q}), \quad \text { for } \quad n>1,
$$

which matches the constant map contribution [20] for $n_{0}^{0}=-\frac{\chi}{2}$. Exponentiating (5.1) one gets $[49,52]$

$$
\mathcal{Z}(\lambda, \underline{t})=\prod_{\beta}\left[\left(\prod_{k=1}^{\infty}\left(1-y^{k} q^{\beta}\right)^{k n_{\beta}^{0}}\right) \prod_{g=1}^{\infty} \prod_{l=0}^{2 g-2}\left(1-y^{g-l-1} q^{\beta}\right)^{(-1)^{g+l}\left({ }^{2 g-2}{ }_{l}\right) n_{\beta}^{g}}\right] .
$$

\subsubsection{Refined BPS invariants}

Let us define as in [42] in analogy with (4.2), (4.4)

$$
F=\sum_{g=0}^{\infty}\left(\epsilon_{1}+\epsilon_{2}\right)^{2 h}\left(\epsilon_{1} \epsilon_{2}\right)^{g-1} F^{(g, h)}(q, Q) .
$$

We decompose the genus $g$ amplitude as

$$
F^{(g, h)}=\sum_{\beta \in H_{2}\left(B_{n}, \mathbb{Z}\right)} F_{\beta}^{(g, h)}(q) Q^{\beta}
$$

then holomorphic anomaly equation for E-strings [43] becomes

$$
\frac{\partial F_{n}^{(g, h)}}{\partial \hat{E}_{2}}=\frac{1}{24} \sum_{h_{1}=0}^{h} \sum_{g_{1}=0}^{g} \sum_{s=1}^{n-1} s(n-s) F_{s}^{\left(g_{1}, h_{1}\right)} F_{n-s}^{\left(g-g_{1}, h-h_{1}\right)}+\frac{n(n+1)}{24} F_{n}^{(g-1, h)}-\frac{n}{24} F_{n}^{(g, h-1)} .
$$

Making an analogous fibre and base separation as in (4.9) of the $E$-string geometry $\frac{1}{2} K 3$ surface with the rational elliptic fibration $\mathcal{E} \rightarrow \mathbb{P}^{1}$, one finds [30] for the massless $E$-string the analogous equation to (4.10), compare [28]

$$
\left(\partial_{E_{2}}+\frac{1}{24}\left[\epsilon_{1} \epsilon_{2}\left(n^{2}+n\right)-\left(\epsilon_{1}+\epsilon_{2}\right)^{2} n\right]\right) Z_{n}^{E-\operatorname{string}}\left(\tau, \epsilon_{1}, \epsilon_{2}\right)=0 .
$$

We note that in the Nekrasov-Shatashvili limit the solution will be a weak Jacobi form. Indeed the index grows linearly as in the $N=4$ case discussed in the introduction. In the general case we expect as solution a meromorphic weak Jacobi theta function. The refined BPS invariants

$$
N_{j_{L}, j_{R}}^{\beta} \in \mathbb{N}_{0} .
$$


are labelled by $\beta \in H_{2}(M, \mathbb{Z})$ and $j_{L}, j_{R}$, the $5 d$ left and right spin

$$
j_{L}, j_{R} \in \frac{1}{2} \mathbb{Z}_{\geq 0}
$$

If we define

$$
[j]_{x}=x^{2 j}+x^{-2 j+2}+\ldots+x^{2 j-2}+x^{2 j}
$$

then (5.1) generalizes with $u=e^{i \epsilon_{1}}$ and $v=e^{i \epsilon_{2}}$ to

$$
\mathcal{F}\left(\epsilon_{1}, \epsilon_{2}, t\right)=\sum_{\beta \in \mathbb{Z}^{r}} \sum_{j_{J}, j_{R}}^{\infty} \sum_{m=1}^{\infty} \frac{(-1)^{2\left(j_{L}+j_{R}\right)} N_{\beta}^{j_{L}, j_{R}}\left[m j_{L}\right]_{(u v)}\left[m j_{R}\right]_{\left(\frac{u}{v}\right)}}{m\left(u^{\frac{m}{2}}-u^{-\frac{m}{2}}\right)\left(v^{\frac{m}{2}}-v^{-\frac{m}{2}}\right)} q^{m \beta},
$$

while $(5.5)$ generalizes to $[14,45]^{30}$

$$
\begin{aligned}
& \mathcal{Z}=\prod_{\beta} \prod_{j_{L / R}=0}^{\infty} \\
& \prod_{m_{L / R}=-j_{L / R}}^{j_{L / R}} \prod_{m_{1}, m_{2}=1}^{\infty}\left(1-\left(\frac{u}{v}\right)^{m_{L}}(u v)^{m_{R}} u^{\left(m_{1}-\frac{1}{2}\right)} v^{\left(m_{2}-\frac{1}{2}\right)} q^{\beta}\right)^{(-1)^{2\left(j_{L}+j_{R}\right)} N_{j_{L} j_{R}}^{\beta}},
\end{aligned}
$$

For the E-string (4.39) generalizes to

$$
\mathcal{Z}_{1}=\sum_{j_{L}, j_{R}} \sum_{n_{e}=0}^{\infty} N_{j_{L}, j_{R}}^{n_{e}, 1}\left[j_{L}\right]_{u}\left[j_{R}\right]_{v} q^{n_{e}}=\frac{E_{4}(q)}{\eta(q)^{8} \prod_{n=1}^{\infty}\left(1-u v q^{n}\right)\left(1-\frac{u q^{n}}{v}\right)\left(1-\frac{q^{n}}{u v}\right)\left(1-\frac{v q^{n}}{u}\right)} .
$$

This can easily seen to be compatible with (5.8). Eqs. (5.8), (5.9) and (5.12) suggest that a refinement of (4.13), (4.10) and (4.39). We will discuss this further in [39].

\subsection{Gopakumar-Vafa invariants from geometry}

\subsubsection{Geometry of curves}

In this section, we describe the geometry of a number of families of curves in the $X_{18}(1,1,1,6,9)$ model, from which a good number of GV invariants can be extracted. For simplicity, we will simply refer to this model as $X$ in this section.

We begin by reviewing some results and notation from [12], which will be used to describe the geometry.

The Calabi-Yau $X$ is described as a blowup of a degree 18 weighted hypersurface in $\mathbb{P}(1,1,1,6,9)$ at its (unique) singular point $x_{1}=x_{2}=x_{3}=0$. As noted earlier, $X$ has Hodge numbers $h^{1,1}=2$ and $h^{2,1}=272$, and Euler characteristic -540 .

The exceptional divisor $E$ is isomorphic to $\mathbb{P}^{2}$. Projection to the first three coordinates of the weighted projective space presents $X$ as a Weierstrass elliptic fibration $\pi: X \rightarrow \mathbb{P}^{2}$. Let $L=\pi^{-1}(\ell)$ be the pullback of a line $\ell \subset \mathbb{P}^{2}$ to $X$, and let $H=3 L+E$. Then

\footnotetext{
${ }^{30}$ See [14] for a mathematical definition of the $N_{j_{L} j_{R}}^{\beta}$ in the local case using the virtual Bialynicki-Birula decomposition of the moduli space Pandharipande-Thomas invariants.
} 
$\pi^{*}\left(x_{1}\right), \pi^{*}\left(x_{2}\right)$, and $\pi^{*}\left(x_{3}\right)$ are sections of $L, \pi^{*}\left(x_{4}\right)$ is a section of $2 H$, and $\pi^{*}\left(x_{5}\right)$ is a section of $3 H$.

For the benefit of those readers more familiar with the toric description, we refer to (2.3) where the two descriptions of the divisors are related: the toric divisor $D_{x_{0}}$ is identified with $E$, the divisor classes of the toric divisors $D_{x_{i}}$ are all equal to $L$ for $i=1,2,3$, the toric divisor $D_{x}$ is in the class $2 H$, and the toric divisor $D_{y}$ is in the class $3 H$.

The Kähler cone of $X$ is generated by $H$ and $L$. The triple intersections are

$$
H^{3}=9, \quad H^{2} L=3, \quad H L^{2}=1, \quad L^{3}=0 .
$$

Dually, the Mori cone is generated by the class of an elliptic fiber $f$ and the class of a line $\tilde{\ell} \subset E \simeq \mathbb{P}^{2}$.

Now, let $C \subset X$ be a connected curve (not necessarily reduced or irreducible, but having no embedded points). The invariants of $C$ are

$$
d_{E}=C \cdot H, \quad d_{B}=C \cdot L, \quad g=p_{a}(C),
$$

where the last is the arithmetic genus of $C$. The curves of fixed $\left(g ; d_{E}, d_{B}\right)$ are parametrized by a Hilbert scheme, and we will be able to describe many of these as well as the corresponding GV invariants.

As a comment on the mathematical rigor of our calculations, we are actually studying PT moduli spaces and computing PT invariants, which are mathematically equivalent to the GV invariants invariants by [67]. However, we will frequently simplify the exposition by using the language of Hilbert schemes as in [50] and then describing corrections required by the use of stable pairs. The reader is referred to [67] for more details, and to [14] for an amplification of the comparison between the methods of [14] and [50].

We say that $\left(d_{E}, d_{B}\right)$ is the degree of $C$, and sometimes use the notation $C_{d_{E}, d_{B}}$ for a curve of degree $\left(d_{E}, d_{B}\right)$. A fiber $f$ has degree $(1,0)$ and a line $\tilde{\ell} \subset E$ has degree $(0,1)$.

We are able to completely describe the moduli space of connected curves of degree $\left(d_{E}, d_{B}\right)$ and genus $g$ for various $d_{E}, d_{B}$, and $g$. In this section we describe the geometric principles that these descriptions are based on. In the next section, we will apply these principles to compute various moduli spaces with $d_{B} \leq 5$ and use this description to compute the associated Gopakumar-Vafa invariants, and make some comments about general $d_{B}$.

Lemma 1. Let $C$ have degree $\left(d_{E}, d_{B}\right)$. Then $\pi(C)$ is a plane curve of degree $d_{B}$, including multiplicity.

Proof. We compute the degree of $\pi(C)$ as

$$
\pi(C) \cdot \ell=C \cdot \pi^{-1}(\ell)=C \cdot L=d_{B},
$$

where $\ell \subset \mathbb{P}^{2}$ is a line.

Corollary 1. A curve of degree $\left(d_{E}, 0\right)$ is a union of $d_{E}$ fibers (including multiplicity).

Proof. By Lemma 1, $\pi(C)$ is a finite point set, hence $C$ is a union of fibers. Since each fiber has degree $(1,0)$, the result follows. 
Lemma 2. If $d_{E}<3 d_{B}$, then for any curve $C$ of degree $\left(d_{E}, d_{B}\right)$ at least one component of $C$ (with its reduced structure) is contained in $E$. In particular, if in addition $C$ is irreducible, then $d_{E}=0$ and $C_{\mathrm{red}} \subset E$, where $C_{\mathrm{red}}$ is the reduced (multiplicity 1) structure on $C$.

Proof. To see this, we first note that if a curve $C^{\prime}$ is not contained in $E$, then $C^{\prime} \cdot E \geq 0$, as the intersection number is just a count of the intersection points of $C^{\prime}$ and $E$ with multiplicity. Then we compute

$$
C \cdot E=C \cdot(H-3 L)=d_{E}-3 d_{B} .
$$

If this is negative, it follows immediately that some component $C^{\prime}$ of $C$ must be contained in $E$. If in addition $C$ is irreducible, it follows that $C_{\text {red }} \subset E$. Since $H \cdot E=0$, it follows that $d_{E}=C \cdot H=0$ as claimed.

Remark. If $C_{\text {red }} \subset E$, it is possible for $C$ itself to not be contained in $E$ due to thickenings in a direction transverse to $E$.

Proposition 1. If $C$ has degree $\left(0, d_{B}\right)$, then its genus satisfies

$$
g \leq \frac{1}{2}\left(d_{B}-1\right)\left(d_{B}-2\right),
$$

with equality holding if and only if $C$ is a plane curve of degree $d_{B}$ after identifying $E$ with $\mathbb{P}^{2}$.

The second half of the proposition says that if we try to thicken components of $C$ outside of $E$, the genus would be less than if we thicken inside $E$.

Proof. We have $C \cdot H=d_{E}=0$. Since $H$ is ample on the weighted hypersurface in $\mathbb{P}(1,1,1,6,9)$ before the blowup, the only way for the intersection $C \cdot H$ to be 0 is for $C_{\text {red }}$ to be contained in $E$. Since any $\mathbb{P}^{2}$ in a Calabi-Yau threefold has a neighborhood which is isomorphic to a neighborhood of $\mathbb{P}^{2}$ inside local $\mathbb{P}^{2}$, the result follows from the corresponding result for local $\mathbb{P}^{2}$.

Remark. We will see in the next subsection that for each $d_{B} \geq 2$, non-reduced curves occur generically in moduli spaces of curves with particular $d_{E}$ and $g$.

We now set out to find the largest possible genus of a connected curve of degree $\left(d_{E}, d_{B}\right)$. Part of our strategy in studying curves is to first study irreducible curves and then study how the components can glue together.

By Lemma 1, a curve $C$ of degree $\left(d_{E}, d_{B}\right)$ is contained in a surface $\pi^{-1}(D)$ where $D \subset E \simeq \mathbb{P}^{2}$ is a plane curve of degree less than or equal to $d_{B}$. The degree will be strictly less than $d_{B}$ if and only if some component of $\pi(C)$ has multiplicity greater than 1 .

By analogy with the case of curves in $\mathbb{P}^{3}$, where the genus of a curve of degree $d$ is maximized when the curve is contained in a plane, one might expect that in our situation the genus is maximized if the degree of $D$ is one, i.e. when $C \subset \pi^{-1}(\ell)$ for some line $\ell \subset E$. This turns out to be the case for irreducible curves with $d_{E}>0$. 
Proposition 2. Suppose $C$ is an irreducible curve of degree $\left(d_{E}, d_{B}\right)$ with $d_{E}>0$. Then

$$
g \leq d_{E} d_{B}-\frac{1}{2}\left(3 d_{B}^{2}-d_{B}-2\right)
$$

with equality holding if and only if $C \subset \pi^{-1}(\ell)$ for some $\ell$. In that case, $C \subset S$ is the zero locus of a section of $\mathcal{O}_{\mathcal{S}}\left(d_{E} L+d_{B} E\right)$. The moduli space of all curves $C$ (not necessarily irreducible) given by all $\ell$ and all sections of $\mathcal{O}_{\mathcal{S}}\left(d_{E} L+d_{B} E\right)$ is a $\mathbb{P}^{d_{E} d_{B}-(1 / 2)\left(3 d_{B}^{2}+d_{B}-4\right)}$ bundle over $\mathbb{P}^{2}$.

Part of the proof involves an analysis of certain curves in surfaces of the form $S=$ $\pi^{-1}(\ell)$ which will be given below. Another part of the proof is analogous to the proof of the Castelnuovo bound for the genus of a curve of fixed degree in $\mathbb{P}^{3}$. The proof itself is omitted.

We now describe curves in a smooth $S=\pi^{-1}(\ell)$.

Suppose that $S \in|L|$, i.e. $S=\pi^{-1}(\ell)$ for some line $\ell \subset \mathbb{P}^{2}$. Then for $C \subset S, \pi(C)$ is either a point $p$ (in which case $C$ is an elliptic fiber of $\pi$, hence completely understood), or $\pi(C)=\ell$. Conversely, if $C$ is any curve in $X$ for which $\pi(C)=\ell$, then $C \subset S=\pi^{-1}(\ell)$.

We now describe all divisors on $S$ associated to line bundles on $S$ which are restrictions of line bundles on $X$. We denote restrictions to $S$ by a subscript, so that the basic divisor classes on $S$ are $E_{S}$ and $L_{S}$. For divisors $D$ and $D^{\prime}$ on $X$ we have for the intersection on $S$ of their restrictions

$$
D_{S} \cdot D_{S}^{\prime}=D \cdot D^{\prime} \cdot L
$$

where the intersection on the right hand side is taken in $X$.

From (5.17), (5.13), and $E=H-3 L$ we get

$$
E_{S}^{2}=-3, E_{S} H_{S}=0, H_{S}^{2}=3, H_{S} L_{S}=1, E_{S} L_{S}=1, L_{S}^{2}=0 .
$$

Let $C \subset S$ be in the class $[C]=d_{E} L_{S}+d_{B} E_{S}$. Then by (5.18) we see that $C \cdot L=d_{B}$ and $C \cdot H=d_{E}$, consistent with our earlier conventions. The moduli of such $C$ in fixed $S$ is a projective space of dimension depending on $\left(d_{E}, d_{B}\right)$ of a genus also depending on $\left(d_{E}, d_{B}\right)$. We now turn to computing the genus and dimension.

For simplicity of exposition, we assume that $S$ is smooth. The adjunction formula says

$$
K_{S}=\mathcal{O}_{S}\left(L_{S}\right)
$$

By the projection formula we get $H^{0}\left(S, K_{S}\right)=H^{0}\left(S, \mathcal{O}_{S}\left(L_{S}\right)\right)=H^{0}\left(\ell, \mathcal{O}_{\ell}(1)\right)$ and so $p_{g}=\operatorname{dim} H^{0}\left(S, K_{S}\right)=2$. Here we are using the language of classical algebraic geometry, where $p_{g}=\operatorname{dim} H^{0}\left(S, K_{S}\right)$ is the geometric genus of a complex surface.

It is not hard to see that $q:=h^{1}\left(S, \mathcal{O}_{S}\right)=0$. So

$$
\chi\left(\mathcal{O}_{S}\right)=1-q+p_{g}=3 .
$$

The genus is given by

$$
g=\frac{1}{2} C\left(C+K_{S}\right)+1=\frac{1}{2}\left(d_{E} L_{S}+d_{B} E_{S}\right)\left(\left(d_{E}+1\right) L_{S}+d_{B} E_{S}\right)+1
$$


which simplifies to

$$
g=d_{E} d_{B}+\frac{1}{2}\left(-3 d_{B}^{2}+d_{B}+2\right) .
$$

The curves $C \subset S$ are a projective space of dimension $h^{0}(S, \mathcal{O}(C))-1$. If $d_{E}>0$ and $d_{B}>1$ then $C-K_{S}=C-L_{S}$ is ample, so by Kodaira vanishing we have $h^{i}(S, \mathcal{O}(C))=0$ for $i>0$. So we only need to compute $\chi(\mathcal{O}(C))$ :

$$
\chi(\mathcal{O}(C))=\frac{1}{2} C\left(C-K_{S}\right)+\chi\left(\mathcal{O}_{S}\right)=d_{E} d_{B}-\frac{1}{2}\left(3 d_{B}^{2}+d_{B}-6\right) .
$$

Since the moduli of these $S$ is just $\mathbb{P}^{2}$ corresponding to the family of lines in $\mathbb{P}^{2}$, the moduli space of such curves $C$ is a $\mathbb{P} \chi(\mathcal{O}(C))-1$-bundle over $\mathbb{P}^{2}$, with $\chi(\mathcal{O}(C))$ given by (5.23).

\subsubsection{Examples and computations}

In this section, we apply the results of the previous section to describe many moduli spaces of curves and compute the associated Gopakumar-Vafa invariants. We denote the moduli space of curves of degree $\left(d_{E}, d_{B}\right)$ and genus $g$ by $\mathcal{M}_{d_{E}, d_{B}}^{g}$. If $\mathcal{M}_{d_{E}, d_{B}}^{g}$ is smooth and there are no curves of degree $\left(d_{E}, d_{B}\right)$ and genus strictly greater than $g$, we have

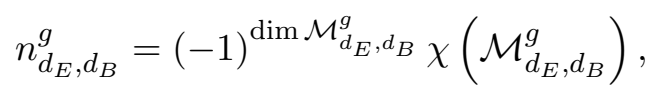

a formula that may need correction as discussed in section 5.2.1.

Now let $\mathcal{C}_{d_{E}, d_{B}}^{g}$ be the universal curve, also supposed smooth, and suppose that there are no other curves of degree $\left(d_{E}, d_{B}\right)$ and genus greater than or equal to $g-1$. Then we have, by [50]

$$
n_{d_{E}, d_{B}}^{g-1}=(-1)^{\operatorname{dim} \mathcal{M}_{d_{E}, d_{B}}^{g}+1}\left(\chi\left(\mathcal{M}_{d_{E}, d_{B}}^{g}\right)+(2 g-2) \chi\left(\mathcal{M}_{d_{E}, d_{B}}^{g}\right)\right) .
$$

This formula may also need correction.

Examples: we begin with $d_{E}=0$ and any $d_{B}>0$. Then we assume $d_{E}>0$ and treat $0 \leq d_{B} \leq 5$ in turn.

$\mathbf{d}_{\mathbf{E}}=\mathbf{0}$. If $g=\left(d_{B}-1\right)\left(d_{B}-2\right) / 2$, then Proposition 1 says that $\mathcal{M}_{0, d_{B}}^{g}$ is identical to the moduli space of plane curves of degree $d_{E}$ in local $\mathbb{P}^{2}$. In particular, the GV invariants $n_{0, d_{B}}^{g}$ for $g \leq p_{a}\left(d_{B}\right)$ are identical with the well-known GV invariants of local $\mathbb{P}^{2}$. This observation provides the geometric verification of the GV invariants in the entire first columns of tables $5-13$.

We will see later that a similar argument can be used to compute or verify many of the GV invariants in some of the subsequent columns.

Now let $C$ have degree $\left(d_{E}, d_{B}\right)$ with $d_{E}>0$ and we set out to bound the genus.

$\mathbf{d}_{\mathbf{B}}=\mathbf{0}$. If $d_{B}=0$, then by Corollary 1 any curve with $d_{B}=0$ is a union of fibers.

It is well known that for an elliptically fibered Calabi-Yau threefold $X$ over a base $B$, the only nonzero Gopakumar-Vafa invariants in multiples of the fiber class $f$ are

$$
\begin{aligned}
& n_{d f}^{1}=\chi(B), \quad d>0 \\
& n_{f}^{0}=-\chi(X) .
\end{aligned}
$$


We therefore get

$$
n_{d_{E}, 0}^{0}=540\left(d_{E}>0\right), \quad n_{1,0}^{1}=3,
$$

in agreement with the $d_{B}=0$ rows of tables $5-13$.

$\mathbf{d}_{\mathbf{B}}=\mathbf{1}$. We compute some Gopakumar-Vafa invariants for $d_{B}=1$ by geometry and always find complete agreement with table 2 and additional calculations we have done beyond the table.

We put $D=\pi(C)$ and note that $D=\ell$ is a line, by Lemma 1 . Then $C$ has a unique irreducible component $C^{\prime}$ mapping by $\pi$ onto $D$. Since any other components of $C$ map to points, they must be unions of fibers, which have $d_{B}=0$. Thus $C$ has $d_{B}=1$. By Proposition 1 we have $g(C) \leq 0$. Hence $g(C)=0$ and $C$ is a line in $E$, again by Proposition 1. Since $C$ has degree $(0,1)$, we see that the remaining components have degree $\left(d_{E}, 0\right)$, hence consists of $d_{E}$ fibers by the $d_{B}=0$ case. Thus $C$ is the union of a line in $E$ and $d_{E}$ fibers, and necessarily has genus $d_{E}$. From this description, we see that $\mathcal{M}_{d_{E}, 1}^{d_{E}}$ is the relative Hilbert scheme of $d_{E}$ points on lines in the plane, a $\mathbb{P}^{d_{E}}$-bundle over $\mathbb{P}^{2}$. Hence by (5.24) we have

$$
\begin{aligned}
n_{d_{E}, 1}^{d_{E}} & =(-1)^{d_{E}} 3\left(d_{E}+1\right) \\
n_{d, 1}^{d_{E}} & =0\left(d<d_{E}\right) .
\end{aligned}
$$

The second equation follows since the same description shows that all curves of degree $(d, 1)$ have genus $d<d_{E}$.

These results agree with those presented in the $d_{B}=1$ row of tables $5-13$. We have also checked these results for much larger values of $d_{E}$, and always find agreement.

$\mathbf{d}_{\mathbf{B}}=\mathbf{2}$. We compute some Gopakumar-Vafa invariants for $d_{B}=2$ by geometry and always find complete agreement with table 3 and additional calculations we have done beyond the table.

We $D=\pi(C)$ and note that $D$ has degree 1 or 2 .

If $D$ is a line $\ell$, then $C \subset \pi^{-1}(\ell)$. By Proposition 2 we get $g=2 d_{E}-4$.

If $D=\ell_{1} \cup \ell_{2}$ is union of lines, then just as in the $d_{B}=1$ case we have that $C$ contains irreducible components $C_{1}$ and $C_{2}$ mapping isomorphically by $\pi$ to $\ell_{1}$ and $\ell_{2}$ respectively. Since each $C_{i}$ has degree $(0,1)$, the remaining components of $C$ have degree $\left(d_{E}, 0\right)$, hence consist of $d_{E}$ fibers. Thus $C$ consists of the reducible conic $C_{1} \cup C_{2}$ in $E$ with $d_{E}$ fibers attached.

If $D$ is an irreducible conic, then $C$ has a unique component $C$ mapping to $C$ with degree 1 . Hence by smoothness of $C$ we see that $C$ is isomorphic to $C$. By Proposition 1, $C$ is a conic in $E$. Since $C$ has degree $(0,2)$, we see just as in the last case that $C$ is the union of $C$ and $d_{E}$ fibers. The last two cases combine into one moduli space of curves, parametrizing conics in $E$ (both smooth and singular) with $d_{E}$ fibers attached. This description shows that moduli space is isomorphic to the relative Hilbert scheme of $d_{E}$ points on plane conics. This space is smooth for $d_{E} \leq 3[50]$. 
Thus the moduli space of all curves of degree $\left(d_{E}, 2\right)$ has two components: the one just described as the relative Hilbert scheme of $d_{E}$ points on plane conics, and the other component parametrizing curves in a surface $S=\pi^{-1}(\ell)$ as in Proposition 2.

The curves of the first component have genus $d_{E}$, and the curves of the second component have genus $2 d_{E}-4$. Thus we have $g \leq \max \left(d_{E}, 2 d_{E}-4\right)$. The maximum is realized on the first component for $d_{E} \leq 4$ and on the second component for $d_{E} \geq 4$.

So for $d_{E} \leq 3$, all curves of genus $g=d_{E}$ are unions of plane conics in $E$ with $d_{E}$ fibers attached, so this moduli space is isomorphic to the relative Hilbert scheme of $d_{E}$ points on plane conics, and this Hilbert scheme is smooth. Computing Euler characteristics exactly as in [50] or [14], we have by (5.24)

$$
n_{0,2}^{0}=-6, n_{1,2}^{1}=15, n_{2,2}^{2}=-36, n_{3,2}^{3}=66
$$

in agreement with the numbers in tables $5-8$. The same argument also shows that all numbers to the left of these numbers are zero.

For $d_{E} \geq 5$, the above discussion shows that curves of genus $g=2 d_{E}-4$ are all contained in surfaces $S=\pi^{-1}(\ell)$ and are described by Proposition 2. So $\mathcal{M}_{d_{E}, 2}^{2 d_{E}-4}$ is a $\mathbb{P}^{2 d_{E}-5}$ bundle over $\mathbb{P}^{2}$, hence by (5.24) we get

$$
n_{d_{E}, 2}^{2 d_{E}-4}=-3\left(2 d_{E}-4\right)
$$

and

$$
n_{d, 2}^{2 d_{E}-4}=0, \quad d<d_{E},
$$

in agreement with the $d_{B}=2$ lines of tables 11-13 for $d_{E} \geq 5$. We have also checked these results for much larger values of $d_{E}$ and always find agreement.

Our last set of checks come from the application of (5.25) to curves of type $((1,2))$. From the genus formula $\max \left(d_{E}, 2 d_{E}-4\right)$ we see that the hypotheses of $(5.25)$ hold if $d_{E}<2 d_{E}-5$, so that $d_{E} \geq 6$. We need to describe the universal curve $\mathcal{C}_{d_{E}, 2}^{2 d_{E}-4}$. Given a point of the universal curve, which we denote by $(C, p)$, we have a natural map $\mathcal{C}_{d_{E}, 2}^{2 d_{E}-4} \rightarrow X$ obtained by forgetting $C$. We claim that the fiber is a $\mathbb{P}^{2 d_{E}-6}$-bundle over $\mathbb{P}^{1}$, hence the universal curve is smooth.

To see this, note that the line $\ell$ for which $C \subset S=\pi^{-1}(\ell)$ must contain the point $q=\pi(p)$. Therefore the lines $\ell$ used to describe curves in the fiber over $p$ form a $\mathbb{P}^{1}$ rather than a $\mathbb{P}^{2}$. The fiber is itself fibered over this $\mathbb{P}^{1}$, and it only remains to find the fiber of this last fibration. Now fixing $\ell$, hence fixing $S$, the possible curves $C$ are those corresponding to sections of $H^{0}\left(S, \mathcal{O}\left(d_{E} L_{S}+d_{B} E_{S}\right)\right)$ which vanish at $p$, which is a hyperplane in $H^{0}\left(S, \mathcal{O}\left(d_{E} L_{S}+d_{B} E_{S}\right)\right)$. So the fiber is a $\mathbb{P}^{2 d_{E}-6}$ rather than a $\mathbb{P}^{2 d_{E}-5}$, as claimed.

We can now apply (5.25) to compute

$$
n_{d_{E}, 2}^{2 d_{E}-5}=(-540)(2)\left(2 d_{E}-5\right)+\left(4 d_{E}-10\right) 3\left(2 d_{E}-4\right), \quad d_{E} \geq 6,
$$

which simplifies to

$$
n_{d_{E}, 2}^{2 d_{E}-5}=12\left(2 d_{B}-5\right)\left(d_{B}-92\right), \quad d_{E} \geq 6,
$$


which agrees with the results in table 3 as well as larger values of $d_{E}$. Furthermore, all numbers to the left of these numbers are zero, which also checks.

The general procedure should now be clear. We let $D=\pi(C)$ and we study the cases where $D$ has irreducible components of each possible degree and multiplicity. In other words the degree $d_{B}$ curve $D$ can split up into components $D_{j}$ of degree $d_{j}$ and multiplicity $m_{j}$. So the possible components of moduli can be indexed by partitions of $d_{B}$ into unordered lists of pairs $\left(\left(d_{j}, m_{j}\right)\right)$ with repetition allowed satisfying

$$
d_{B}=\sum_{j} m_{j} d_{j}
$$

Correspondingly, $C$ splits up into a union of components $C_{j}$, mapping by $\pi$ to $D_{j}$ with multiplicity $m_{j}$, possibly together with fibers. We investigate the maximum genus of each $C_{j}$. The genus of $C$ can then be maximized by configuring the curves $C_{j}$ and any fibers so that they intersect in as many points (including multiplicity) as possible.

For $d_{B}=1$, only the partition $((1,1))$ is possible. For $d_{B}=2$ we could have either $((2,1)),((1,2))$, or $((1,1),(1,1))$, and all cases occurred in our discussion above.

The analysis gets more intricate for each degree. Rather than carry out this program for $d_{E} \leq 5$, we content ourselves with presenting some Gopakumar-Vafa invariants that can be computed by these methods, giving the corresponding $\left(\left(d_{j}, m_{j}\right)\right)$, and matching to the tables.

For any $d_{B} \geq 2$ some strata combine naturally, as we already saw for $d_{B}=2$. Let $d_{B}=\sum_{j=1}^{k}$ be a partition of $d_{B}$ and consider the list $\left(\left(d_{j}, 1\right)_{j=1}^{k}\right)$. These strata combine to describe the Hilbert scheme of $d_{E}$ points on plane curves of degree $d_{B}$. The stratification is just an artifact of our method, corresponding to splitting up the moduli space according to the degrees of the components of the plane curve.

$\mathbf{d}_{\mathbf{B}}=\mathbf{3}$. We compute some Gopakumar-Vafa invariants for $d_{B}=3$ by geometry and always find complete agreement with table 4 and additional calculations we have done beyond the table.

In this case, the possible lists are $((1,1),(1,1),(1,1)),((3,1)),((1,3)),((2,1),(1,1))$, $((1,2)$, or $(1,1))$,

- $((3,1)),((2,1),(1,1))$, and $((1,1),(1,1),(1,1))$ combine to give plane cubics union $d_{E}$ fibers, with moduli space the relative Hilbert scheme of $d_{E}$ points on plane cubics, which are curves of genus $d_{E}+1$.

- $((1,3))$ are curves in a surface of the form $S=\pi^{-1}(\ell)$, which have genus $3 d_{E}-11$ by Proposition 2.

- $((1,2),(1,1))$ is the union of a curve in a surface of the form $S=\pi^{-1}(\ell)$, a line in $E$, and possibly some fibers. It is not hard to see by extending the analysis below that the maximum genus is attained when there are no additional fibers. So $C$ is the union of a curve $C_{d_{E}, 2}$ in $S$ and a line $C_{0,1} \subset E$ (which has degree $(0,1)$ ). Here and in the sequel, a subscript denotes the degree of a curve. We have already seen that $C_{d_{E}, 2}$ has genus $2 d_{E}-4$. 
The genus of $C$ is therefore $\left(2 d_{E}-4\right)+m-1$, where $m$ is the number of intersection points $Z$ of $C_{d_{E}, 2}$ and $\ell$. We claim that either $m=1$ or we are actually in the $((1,3))$ case. Since $C_{0,1} \subset E$ we see that $Z \subset C_{d_{E}, 2} \cap E$. Since $S \cap E$ is a line, it follows that if $m \geq 2$, then $C_{0,1}$ must be equal to that line. In that case $C \subset S$, and we are really in the $((1,3))$ case. So $m=1$ and $C$ has genus $2 d_{E}-4$.

Combining the three cases, we have $g \leq \max \left(d_{E}+1,3 d_{E}-11,2 d_{E}-4\right)$.

The maximum genus is attained in the first case for $d_{E} \leq 5$, in the second case for $d_{E} \geq 7$, and in the third case for $5 \leq d_{E} \leq 7$.

If $d_{E} \leq 4$, then the maximum genus $d_{E}+1$ can only be attained in the first case, and $\mathcal{M}_{d_{E}, 3}^{d_{E}+1}$ is the relative Hilbert scheme of $d_{E}$ points on plane cubics, which is smooth by [50]. So we compute from the relative Hilbert scheme

$$
n_{0,3}^{1}=-10, n_{1,3}^{2}=27, n_{2,3}^{3}=-72, n_{3,3}^{4}=154, n_{4,3}^{5}=-306 .
$$

This agrees with tables $6-10$. In addition, our now-familiar argument shows that all numbers to the left of these numbers in the tables are zero, in agreement with the tables.

If $d_{E} \geq 8$, then the maximum genus $3 d_{E}-11$ can only be attained in the second case $((1,3))$, and $\mathcal{M}_{d_{E}, 3}^{3 d_{E}-11}$ is a $\mathbb{P}^{3 d_{E}-13}$-bundle over $\mathbb{P}^{2}$ by Proposition 2 . Hence, for $d_{E} \geq 8$ we have

$$
n_{d_{E}, 3}^{3 d_{E}-11}=(-1)^{d_{E}+1} 9\left(d_{E}-4\right) .
$$

For these $d_{E}$ we also have $n_{d, 3}^{3 d_{E}-11}=0$ for $d<d_{E}$. We have checked these results for large values of $d_{E}$ and always find agreement.

For $d_{E}=6$, the maximum genus $2 d_{E}-4=8$ can only be realized in the third case $((1,2),(1,1))$. By the discussion above, we have $C=C_{6,2} \cup C_{0,1}$, where $C_{6,2}$ lies in a surface $S=\pi^{-1}(\ell)$ and the line $C_{0,1}$ intersects $C_{6,2}$ in exactly one point. But $C_{6,2} \cdot E=0$ by (5.16). On the other hand, $C_{6,2}$ cannot be disjoint from $E$, since it intersects $C_{0,1} \subset E$ nontrivially. Therefore $C_{6,2}$ has a component which lies entirely in $E$, necessary a line or a conic. If it contains a conic plane curve, then since it also contains the line $C_{0,1}$ then it contains a (reducible) cubic plane curve. So we are really in the case of a cubic with $d_{E}$ fibers already considered. So $C_{6,2}$ contains a line. The remaining components are a curve $C_{6,1}$, which by the $d_{B}=1$ case are a line and 6 fibers. If the two lines are distinct, then they form a conic and we are in the previous case. Otherwise the line has multiplicity 2 , and the curve $C_{6,2}$ is the union of the line $E_{S}$ with multiplicity 2 (in $S$ ) and 6 fibers. The line $C_{0,1}$ is arbitrary. So $\mathcal{M}_{6,3}^{8}$ is the product of $\mathbb{P}^{2}$ and the relative Hilbert scheme of 6 points in lines in $\mathbb{P}^{2}$. Since this relative Hilbert scheme is a $\mathbb{P}^{6}$-bundle over $\mathbb{P}^{2}$, this gives

$$
n_{6,3}^{8}=63,
$$

in agreement with table 13. Furthermore, all of the numbers to the left are zero, again in agreement.

We can also apply $(5.25)$ for curves of type $((1,3))$, which have genus $3 d_{E}-11$. The hypotheses hold if $d_{E} \geq 9$ and then we get

$$
n_{d_{E}, 3}^{3 d_{E}-12}=(-1)^{d_{E}}\left((-540)(2)\left(3 d_{E}-13\right)+\left(6 d_{E}-24\right)(3)\left(3 d_{E}-12\right)\right),
$$


which simplifies to

$$
n_{d_{E}, 3}^{3 d_{E}-12}=(-1)^{d_{E}}(54)\left(d^{2}-68 d+276\right), \quad d_{E} \geq 9,
$$

which agrees with the results in table 4 as well as larger values of $d_{E}$. Furthermore, all numbers to the left of these numbers are zero, which also checks.

$\mathbf{d}_{\mathbf{B}}=4$. We compute some Gopakumar-Vafa invariants for $d_{B}=4$ by geometry and always find complete agreement with table 14 and additional calculations we have done beyond the table. By now we have provided enough examples that we can simply present our results and the reader will be able to check details.

Combining the cases $((4,1)),((3,1),(1,1)),((2,1),(2,1)),((2,1),(1,1),(1,1))$, and $((1,1),(1,1),(1,1),(1,1))$, we consider plane quartic curves in $E$ with $d_{E}$ fibers attached. These curves have genus $d_{E}+3$. If $d_{E} \leq 6$, any curve of degree $\left(d_{E}, 4\right)$ and genus $d_{E}+3$ can be seen to be of this type, and furthermore there are no curves of that degree and higher genus. We conclude that $\mathcal{M}_{d_{E}, 4}^{d_{E}+3}$ is the relative Hilbert scheme of $d_{E}$ points on plane quartic curves, a $\mathbb{P}^{14-d_{E}}$ bundle over $\operatorname{Hilb}^{d_{E}}\left(\mathbb{P}^{2}\right)$, and is smooth for $d_{E} \leq 5$ [50]. We therefore get

$$
n_{d_{E}, 4}^{d_{E}+3}=(-1)^{d_{E}}\left(15-d_{E}\right) \chi\left(\operatorname{Hilb}^{d_{E}}\left(\mathbb{P}^{2}\right)\right),
$$

hence by computing Euler characteristics

$$
n_{0,4}^{3}=15, n_{1,4}^{4}=-42, n_{2,4}^{5}=117, n_{3,4}^{6}=-264, n_{4,4}^{7}=561, n_{5,4}^{8}=-1080 .
$$

The same argument confirms in the usual way that all numbers to the left of these are zero, in agreement with the tables.

For curves of type $((1,4))$, i.e. curves contained in a surface $S=\pi^{-1}(\ell)$, we have $g=4 d_{E}-21$, and $\mathcal{M}_{d_{E}, 4}^{4 d_{E}-21}$ is a $\mathbb{P}^{4 d_{E}-24}$-bundle over $\mathbb{P}^{2}$ by Proposition 2 . If $d_{E} \geq 11$, then any curve of this degree and genus is of this type, and there are no curves of this degree and higher genus. This gives

$$
n_{d_{E}, 4}^{4 d_{E}-21}=3\left(4 d_{E}-23\right)=12 d_{E}-69, d_{E} \geq 11
$$

in complete agreement with our calculations. Also $n_{d, 4}^{4 d_{E}-21}=0$ for $d<d_{E}$.

For $d_{E}=7$ we have curves of type $((1,2),(2,1))$, which consist of a curve $C_{7,2}$ of degree $(7,2)$ union a conic $C_{0,2} \subset E$. The curve $C_{7,2}$ lies in a surface $S=\pi^{-1}(\ell)$ and has genus $2 d_{E}-4=10$. Since $C_{0,2}$ is to meet $C_{7,2}$ in two points but $C_{7,2} \cdot E=1$, we see that $C_{7,2}$ must contain a line in $E$. Repeating an argument from the $d_{B}=3$ case, we further see that $C_{7,2}$ must contain a line doubled in $S$ together with $d_{E}$ fibers. Our geometric description shows that the moduli space of $C_{7,2}$ is the relative Hilbert scheme of 7 points on lines, a $\mathbb{P}^{7}$-bundle over $\mathbb{P}^{2}$. There are no curves of degree $(7,2)$ and higher genus, so we can now multiply be the $\mathbb{P}^{5}$ moduli of $C_{0,2}$ and compute the Euler characteristic, giving

$$
n_{7,4}^{16}=144
$$


in agreement with our other calculations. In addition we have $n_{d, 4}^{16}=0$ for $d<7$, also in agreement.

We can also apply $(5.25)$ for curves of type $((1,4))$, which have genus $4 d_{E}-21$. The hypotheses hold if $d_{E} \geq 12$ and then we get

$$
n_{d_{E}, 4}^{4 d_{E}-22}=-\left((-540)(2)\left(4 d_{E}-24\right)+\left(8 d_{E}-44\right)(3)\left(4 d_{E}-23\right)\right),
$$

which simplifies to

$$
n_{d_{E}, 4}^{4 d_{E}-22}=-54\left(d^{2}-68 d+276\right), \quad d_{E} \geq 12,
$$

which agrees with the results in table 14 as well as larger values of $d_{E}$. Furthermore, all numbers to the left of these numbers are zero, which also checks.

$\mathbf{d}_{\mathbf{B}}=\mathbf{5}$. We compute some Gopakumar-Vafa invariants for $d_{B}=5$ by geometry and always find complete agreement with table 15 and additional calculations we have done beyond the table.

We first consider the curves which are unions of plane quintics and $d_{E}$ fibers, which have genus $d_{E}+6$. We also compute that for $d_{E} \leq 7$, all curves of degree $\left(d_{E}, 5\right)$ and genus $d_{E}+6$ are of this type. Therefore $\mathcal{M}_{d_{E}, 5}^{d_{E}+6}$ is the relative Hilbert scheme of $d_{E}$ points on plane quintics, which is smooth for $d_{E} \leq 6$. We also compute that there are no curves of that degree and higher genus. Since $\mathcal{M}_{d_{E}, 5}^{d_{E}+6}$ is a $\mathbb{P}^{20-d_{E}}$-bundle over $\operatorname{Hilb}^{d_{E}}\left(\mathbb{P}^{2}\right)$, we get

$$
n_{d_{E}, 5}^{d_{E}+6}=(-1)^{d_{E}}\left(21-d_{E}\right) \chi\left(\operatorname{Hilb}^{d_{E}}\left(\mathbb{P}^{2}\right)\right) \quad d_{E} \leq 6,
$$

or

$$
n_{0,5}^{6}=21, n_{1,5}^{7}=-60, n_{2,5}^{8}=171, n_{3,5}^{9}=-396, n_{4,5}^{10}=867, n_{5,5}^{11}=-1728, n_{6,5}^{12}=3315 .
$$

in complete agreement with our calculations. Also the Gopakumar-Vafa invariants to the left of these numbers are all zero, also in agreement.

For curves of type $((1,5))$, i.e. those contained in a surface $S=\pi^{-1}(\ell)$ we have $g=$ $5 d_{E}-34$ and moduli space a $\mathbb{P}^{5 d_{E}-38}$-bundle over $\mathbb{P}^{2}$. For $d_{E} \geq 14$ we compute that all curves of degree $\left(d_{E}, 5\right)$ are of this type, and that there are no curves of higher genus. This gives

$$
n_{5, d_{E}}^{5 d_{E}-34}=(-1)^{d_{E}} 3\left(5 d_{E}-37\right) \quad d_{E} \geq 14 .
$$

in complete agreement with our calculations. Also the Gopakumar-Vafa invariants to the left of these numbers are all zero, also in agreement.

We can also apply $(5.25)$ for curves of type $((1,5))$, which have genus $5 d_{E}-34$. The hypotheses hold if $d_{E} \geq 15$ and then we get

$$
n_{d_{E}, 5}^{5 d_{E}-35}=(-1)^{d_{E}+1}\left((-540)(2)\left(5 d_{E}-38\right)+\left(10 d_{E}-70\right)(3)\left(5 d_{E}-37\right)\right),
$$

which simplifies to

$$
n_{d_{E}, 5}^{5 d_{E}-35}=(-1)^{d_{E}+1}(30)\left(5 d_{B}^{2}-252 d_{B}+1627\right), \quad d_{E} \geq 15,
$$

which agrees with the results in table 14 as well as larger values of $d_{E}$. Furthermore, all numbers to the left of these numbers are zero, which also checks. 


\section{Acknowledgments}

We thanks Babak Haghighat, Guglielmo Lockhart and Cumrun Vafa for discussions about related structures in M- and E-string theories and Martin Westerholt-Raum and Don Zagier for crucial comments on Siegel modular- and weak Jacobi forms. We like to thanks Gaetan Borot, Hans Jockers and Marcos Mariño for comments on the draft. This work started when $\mathrm{MH}$ was affiliated with Kavli Institute for the Physics and Mathematics of the Universe (Kavli IPMU). He thanks Kavli IPMU for supports. MH also thanks the BCTP, the HCM and the organizers of "The 2nd Workshop on Developments in M-theory" in Korea for hospitality. MH is supported by the "Young Thousand People" plan by the Central Organization Department in China, and by the Natural Science Foundation of China. S.K. is supported by NSF DMS-12-01089. A.K. thanks for support by KL 2271/1-1 and NSF DMS-11-01089.

\section{A Gopakumar-Vafa invariants}

The involution symmetry of the topological strings on elliptic Calabi-Yau manifolds restricts the number of the coefficients in the ambiguity to roughly one fourth. This allows us to extract the higher genus invariants to sufficient genus $g \leq 8$ to test the conjectures about the even weak Jacobi-Forms in reasonable detail ${ }^{31}$

\begin{tabular}{|c|c|c|c|c|c|c|c|}
\hline$d_{B} \backslash d_{E}$ & 0 & 1 & 2 & 3 & 4 & 5 & 6 \\
\hline 0 & & 540 & 540 & 540 & 540 & 540 & 540 \\
\hline 1 & 3 & -1080 & 143370 & 204071184 & 21772947555 & 1076518252152 & 33381348217290 \\
\hline 2 & -6 & 2700 & -574560 & 74810520 & -49933059660 & 7772494870800 & 31128163315047072 \\
\hline 3 & 27 & -17280 & 5051970 & -913383000 & 224108858700 & -42712135606368 & 4047949393968960 \\
\hline 4 & -192 & 154440 & -57879900 & 13593850920 & -2953943334360 & 603778002921828 & -90433961251273800 \\
\hline 5 & 1695 & -1640520 & 751684050 & -218032516800 & 51350781706785 & -11035406089270080 & 2000248139674298880 \\
\hline
\end{tabular}

Table 5. The GV invariants $n_{\left(d_{E}, d_{B}\right)}^{g}$ for genus $g=0$ for the elliptic Calabi-Yau $X(1,1,1,6,9)$.

\begin{tabular}{|c|c|c|c|c|c|c|c|}
\hline$d_{B} \backslash d_{E}$ & 0 & 1 & 2 & 3 & 4 & 5 & 6 \\
\hline 0 & & 3 & 3 & 3 & 3 & 3 & 3 \\
\hline 1 & 0 & -6 & 2142 & -280284 & -408993990 & -44771454090 & -2285308753398 \\
\hline 2 & 0 & 15 & -8574 & 2126358 & 521856996 & 1122213103092 & 879831736511916 \\
\hline 3 & -10 & 4764 & -1079298 & 152278986 & -16704086880 & -3328467399468 & 1252978673852088 \\
\hline 4 & 231 & -154662 & 48907815 & -9759419622 & 1591062421074 & -186415241060547 & 8624795298947118 \\
\hline 5 & -4452 & 3762246 & -1510850250 & 385304916960 & -76672173887766 & 12768215950604064 & -1663415916220743876 \\
\hline
\end{tabular}

Table 6. The GV invariants $n_{\left(d_{E}, d_{B}\right)}^{g}$ for genus $g=1$ for the elliptic Calabi-Yau $X(1,1,1,6,9)$.

\footnotetext{
${ }^{31}$ As we explained it is in principle possible to evaluate them to genus 189 . The genus $g=0$ invariants agree with [32], the $g \leq 1$ invariants with [12] and the invariants $g \leq 3$ invariants with [3].
} 


\begin{tabular}{|c|c|c|c|c|c|c|c|}
\hline$d_{B} \backslash d_{E}$ & 0 & 1 & 2 & 3 & 4 & 5 & 6 \\
\hline 0 & & 0 & 0 & 0 & 0 & 0 & 0 \\
\hline 1 & 0 & 0 & 9 & -3192 & 412965 & 614459160 & 68590330119 \\
\hline 2 & 0 & 0 & -36 & 20826 & -5904756 & -47646003780 & -80065270602672 \\
\hline 3 & 0 & 27 & -16884 & 4768830 & -818096436 & 288137120463 & 67873415627151 \\
\hline 4 & -102 & 57456 & -15452514 & 2632083714 & -320511624876 & 18550698291252 & 780000198300540 \\
\hline 5 & 5430 & -4032288 & 1430896428 & -323858122812 & 55058565096630 & -7249216518163620 & 691264676523200805 \\
\hline
\end{tabular}

Table 7. The GV invariants $n_{\left(d_{E}, d_{B}\right)}^{2}$ for the elliptic Calabi-Yau $X(1,1,1,6,9)$. Note that $n_{g>1}^{\left(0, d_{2}\right)}=$ $0, \forall d_{2} \in \mathbb{N}$. We therefore omit the $\left(0, d_{2}\right)$ line below.

\begin{tabular}{|c|c|c|c|c|c|c|c|}
\hline$d_{B} \backslash d_{E}$ & 0 & 1 & 2 & 3 & 4 & 5 & 6 \\
\hline 1 & 0 & 0 & 0 & -12 & 4230 & -541440 & -820457286 \\
\hline 2 & 0 & 0 & 0 & 66 & -45729 & 627574428 & 3776946955338 \\
\hline 3 & 0 & 0 & -72 & 48036 & -14756490 & 297044064 & -7900517344212 \\
\hline 4 & 15 & -7236 & 1638918 & -226431351 & 20419274259 & -719284158099 & 236091664016826 \\
\hline 5 & -3672 & 2417742 & -764921214 & 154856849136 & -22866882491772 & 2493418732350750 & -194361733345447458 \\
\hline
\end{tabular}

Table 8. The GV invariants $n_{\left(d_{E}, d_{B}\right)}^{3}$ for the elliptic Calabi-Yau $X(1,1,1,6,9)$.

\begin{tabular}{|c|c|c|c|c|c|c|c|}
\hline$d_{B} \backslash d_{E}$ & 0 & 1 & 2 & 3 & 4 & 5 & 6 \\
\hline 1 & 0 & 0 & 0 & 0 & 15 & -5256 & 665745 \\
\hline 2 & 0 & 0 & 0 & 0 & -132 & -453960 & -95306132778 \\
\hline 3 & 0 & 0 & 0 & 154 & -110574 & 38259441 & 218140445904 \\
\hline 4 & 0 & -42 & 26946 & -7824888 & 1386011568 & -172919782116 & -4345528029372 \\
\hline 5 & 1386 & -819123 & 232934157 & -42321589218 & 5500907292240 & -520718843839590 & 38245592568676608 \\
\hline
\end{tabular}

Table 9. The GV invariants $n_{\left(d_{E}, d_{B}\right)}^{4}$ for the elliptic Calabi-Yau $X(1,1,1,6,9)$.

\begin{tabular}{|c|c|c|c|c|c|c|c|}
\hline$d_{B} \backslash d_{E}$ & 0 & 1 & 2 & 3 & 4 & 5 & 6 \\
\hline 1 & 0 & 0 & 0 & 0 & 0 & -18 & 6270 \\
\hline 2 & 0 & 0 & 0 & 0 & 0 & -5031 & 1028427996 \\
\hline 3 & 0 & 0 & 0 & 0 & -306 & 247014 & -2562122952 \\
\hline 4 & 0 & 0 & 117 & -81225 & 26211942 & -5223900087 & 1263109811373 \\
\hline 5 & -270 & 144414 & -36870264 & 5929743618 & -665294451264 & 53375661928620 & -3651471177372864 \\
\hline
\end{tabular}

Table 10. The GV invariants $n_{\left(d_{E}, d_{B}\right)}^{5}$ for the elliptic Calabi-Yau $X(1,1,1,6,9)$.

\begin{tabular}{|c|c|c|c|c|c|c|c|c|}
\hline$d_{B} \backslash d_{E}$ & 0 & 1 & 2 & 3 & 4 & 5 & 6 & 7 \\
\hline 1 & 0 & 0 & 0 & 0 & 0 & 0 & 21 & -7272 \\
\hline 2 & 0 & 0 & 0 & 0 & 0 & -18 & -771642 & -147864402162 \\
\hline 3 & 0 & 0 & 0 & 0 & 0 & 612 & 1401468 & 502063861662 \\
\hline 4 & 0 & 0 & 0 & -264 & 200430 & -70438068 & 9510828972 & -29413672557570 \\
\hline 5 & 21 & -9972 & 2156373 & -268703481 & 18682746903 & -182455706016 & -81900631565910 & 22514515679407491 \\
\hline
\end{tabular}

Table 11. The GV invariants $n_{\left(d_{E}, d_{B}\right)}^{6}$ for the elliptic Calabi-Yau $X(1,1,1,6,9)$. 


\begin{tabular}{|c|c|c|c|c|c|c|c|c|}
\hline$d_{B} \backslash d_{E}$ & 0 & 1 & 2 & 3 & 4 & 5 & 6 & 7 \\
\hline 1 & 0 & 0 & 0 & 0 & 0 & 0 & 0 & -24 \\
\hline 2 & 0 & 0 & 0 & 0 & 0 & 0 & 17386 & -7224 \\
\hline 3 & 0 & 0 & 0 & 0 & 0 & 0 & 170978160 & 605964021294 \\
\hline 4 & 0 & 0 & 0 & 0 & 561 & -447903 & -4962183570 \\
\hline 5 & 0 & -60 & 38340 & -10994520 & 1895073858 & -2177735855972 & 16072935664050 & -1937578925283840 \\
\hline 6 & 27538 & -16386600 & 4710791727 & -868872423987 & 115076024047737 & -11554540079426667 & 915758222342784613 & -59159328867116232828 \\
\hline
\end{tabular}

Table 12. The GV invariants $n_{\left(d_{E}, d_{B}\right)}^{7}$ for $X(1,1,1,6,9)$.

\begin{tabular}{|c|c|c|c|c|c|c|c|c|c|}
\hline$d_{B} \backslash d_{E}$ & 0 & 1 & 2 & 3 & 4 & 5 & 6 & 7 & 8 \\
\hline 1 & 0 & 0 & 0 & 0 & 0 & 0 & 0 & 0 & 27 \\
\hline 2 & 0 & 0 & 0 & 0 & 0 & 0 & -24 & -995490 & -203754011670 \\
\hline 3 & 0 & 0 & 0 & 0 & 0 & 0 & 63 & 3396663 & 11118565777779 \\
\hline 4 & 0 & 0 & 0 & 0 & 0 & -1080 & 951204 & -6600292956 & -74890630203552 \\
\hline 5 & 0 & 0 & 171 & -119415 & 38611944 & -7672460076 & 10568533387755 & -49874149196514 & 29707605109699254 \\
\hline 6 & -5310 & 2949516 & -785916540 & $132969 \cdot 10^{7}$ & $-159045 \cdot 10^{8}$ & $14105 \cdot 10^{11}$ & $-952701 \cdot 10^{11}$ & $471505 \cdot 10^{13}$ & $-112148 \cdot 10^{15}$ \\
\hline
\end{tabular}

Table 13. The GV invariants $n_{\left(d_{E}, d_{B}\right)}^{8}$ for $X(1,1,1,6,9)$. For last 6 numbers we give only 6 significant digits.

\section{B Derivation of the involution symmetry on the propagators}

For the compact models we have the an-holomorphic propagators $S^{i j}, S^{i}, S$, where $i, j$ runs over the complex structure moduli. It is convenient to make a change of variables with the derivative of the Kähler potential $K_{i}=\partial_{i} K$ by the following

$$
S^{i j} \rightarrow S^{i j}, \quad S^{i} \rightarrow S^{i}-S^{i j} K_{j}, \quad S \rightarrow S-S^{i} K_{i}+\frac{1}{2} S^{i j} K_{i} K_{j}
$$

In the following we refer to the propagators $S^{i j}, S^{i}, S$ as the ones after the change of variables in [2].

The propagators are defined by relating their anti-holomorphic derivatives to three point couplings. One can integrate these relations and also special geometry relation, and gets [2]

$$
\begin{aligned}
\Gamma_{i j}^{k} & =\delta_{i}^{k} K_{j}+\delta_{j}^{k} K_{i}-C_{i j l} S^{k l}+s_{i j}^{k}, \\
\partial_{i} S^{j k} & =C_{i m n} S^{m j} S^{n k}+\delta_{i}^{j} S^{k}+\delta_{i}^{k} S^{j}-s_{i m}^{j} S^{m k}-s_{i m}^{k} S^{m j}+h_{i}^{j k}, \\
\partial_{i} S^{j} & =C_{i m n} S^{m j} S^{n}+2 \delta_{i}^{j} S-s_{i m}^{j} S^{m}-h_{i k} S^{k j}+h_{i}^{j}, \\
\partial_{i} S & =\frac{1}{2} C_{i m n} S^{m} S^{n}-h_{i j} S^{j}+h_{i}, \\
\partial_{i} K_{j} & =K_{i} K_{j}-C_{i j n} S^{m n} K_{m}+s_{i j}^{m} K_{m}-C_{i j k} S^{k}+h_{i j} .
\end{aligned}
$$

Here the holomorphic ambiguities $s_{i j}^{k}, h_{i}^{j k}, h_{i j}, h_{i}^{j}, h_{i}$ are some rational functions from the integration constants of the anti-holomorphic derivatives. We can compute the Kahler potential $K$ and Christoffel connections $\Gamma_{i j}^{k}$ in the holomorphic limit from Picard-Fuchs equations. There is freedom to choose some of the ambiguities such that the set of equations has a consistent solution for the other ambiguities and the propagators $S^{i j}, S^{i}, S$. 


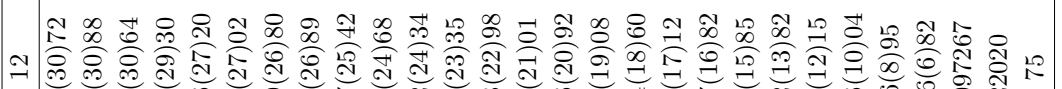

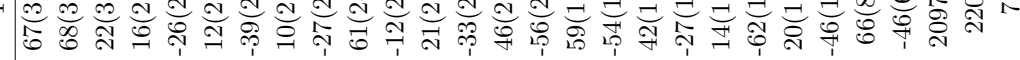

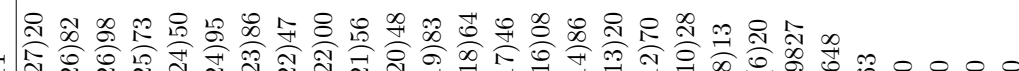

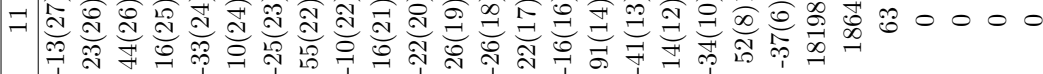
\& $\&$ \&

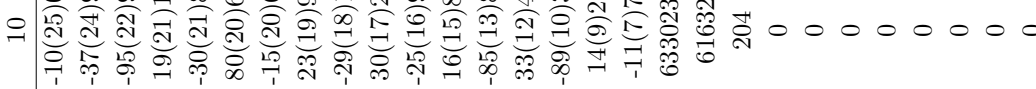

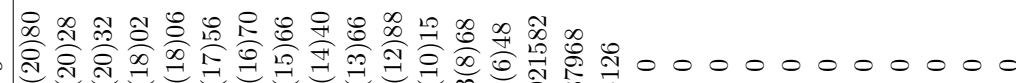

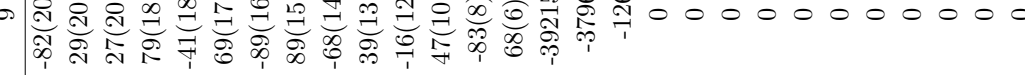

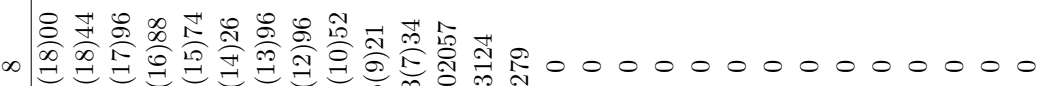

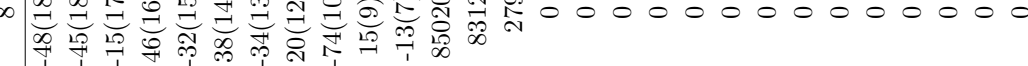

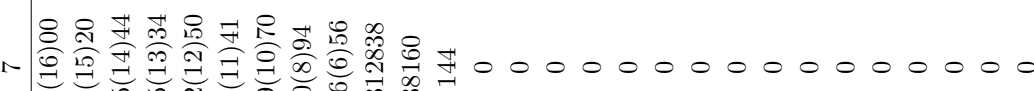

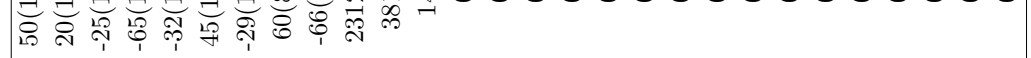

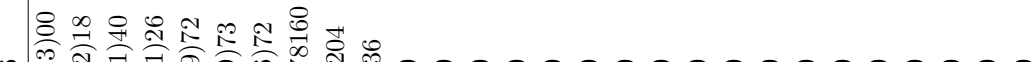

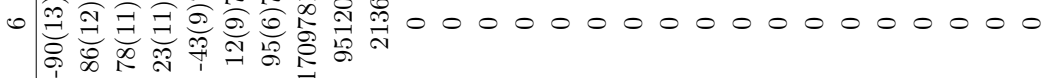

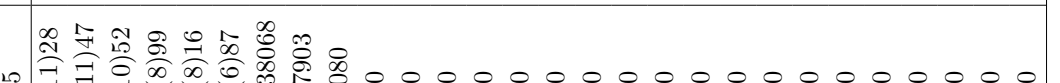
20

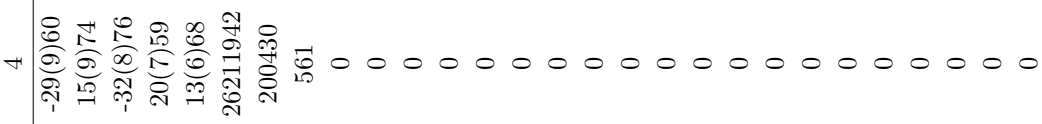

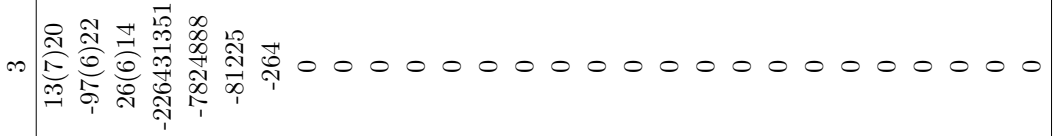
N

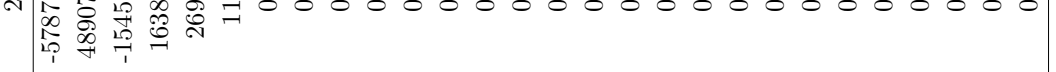

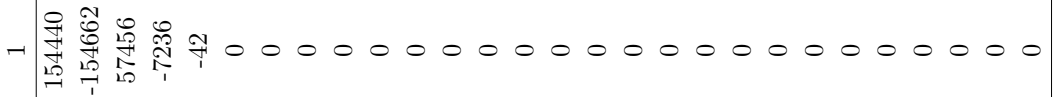

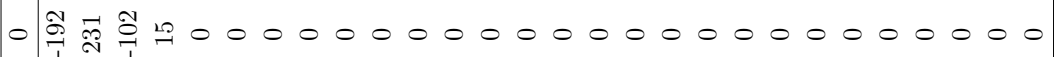

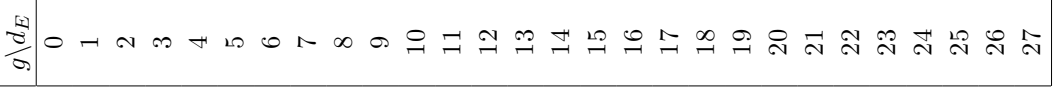




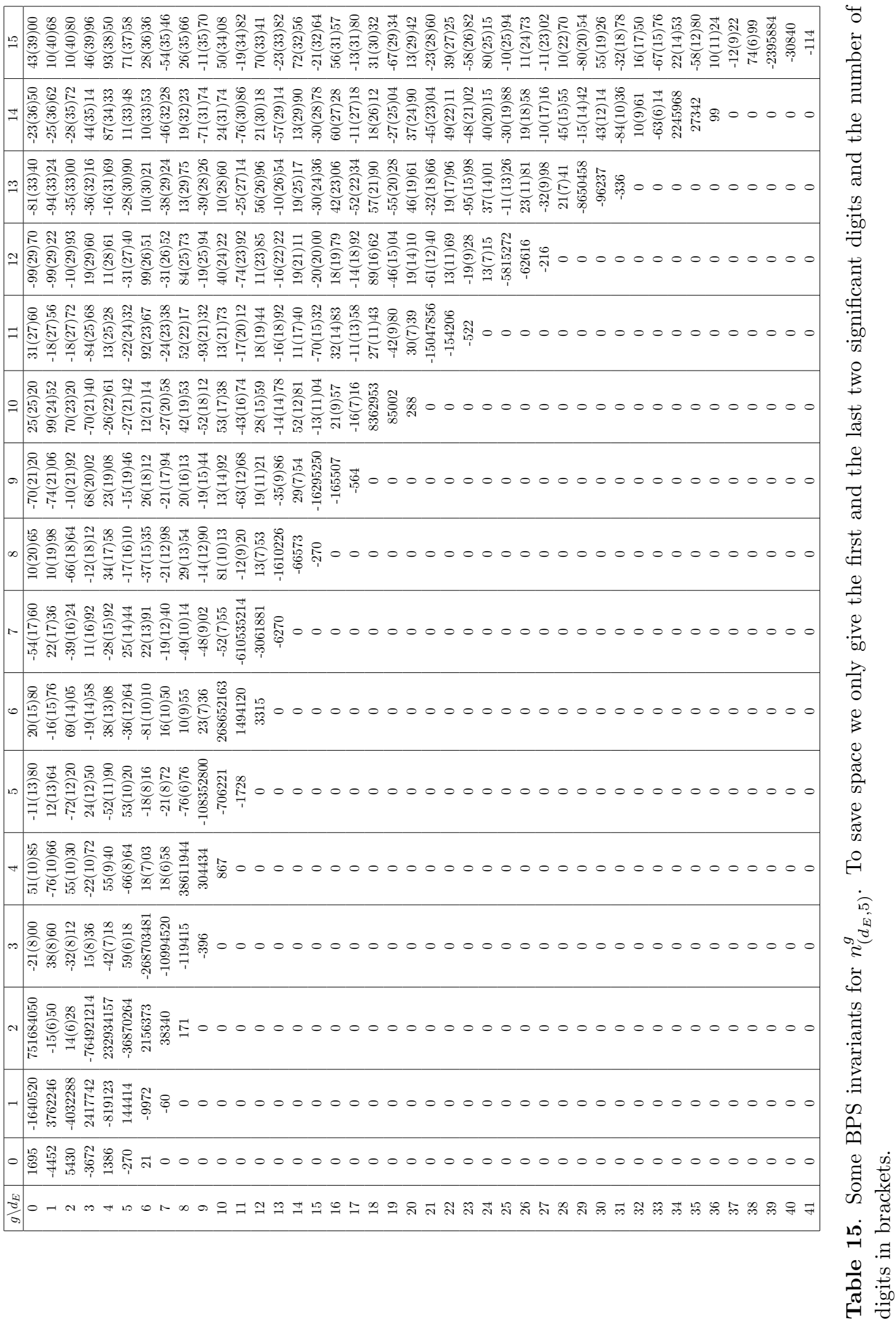


We first discuss the gauge choice made in [3], which are the following

$$
\begin{aligned}
& s_{11}^{1}=-\frac{2}{z_{1}}, \quad s_{12}^{1}=-\frac{1}{3 z_{2}}, \quad s_{22}^{1}=0, \\
& s_{11}^{2}=0, \quad s_{12}^{2}=0, \quad s_{22}^{2}=-\frac{4}{3 z_{2}}, \\
& h_{1}^{11}=z_{1}\left[\frac{1}{9}-48 z_{1}+\frac{5}{6} z_{2}-540 z_{1} z_{2}\right], \quad h_{1}^{12}=z_{2}\left[-\frac{5}{108}-\frac{5}{4} z_{2}+20 z_{1}+540 z_{1} z_{2}\right] \text {, } \\
& h_{1}^{22}=-60 z_{2}^{2}\left(1+27 z_{2}\right), \quad h_{2}^{11}=-60 z_{1}^{3}, \\
& h_{2}^{12}=z_{1}\left[\frac{1}{9}+\frac{5}{12} z_{2}-48 z_{1}\right], \quad h_{2}^{22}=z_{2}\left[-\frac{23}{54}+40 z_{1}-\frac{5}{2} z_{2}-540 z_{1} z_{2}\right] \text {, } \\
& h_{1}^{1}=\frac{155}{27} z_{1}-\frac{25}{1296} z_{2}+50 z_{1} z_{2}, \quad h_{1}^{2}=0, \\
& h_{2}^{1}=-\frac{5}{18} z_{1}+120 z_{1}^{2}, \quad h_{2}^{2}=\frac{155}{27} z_{1}+\frac{1055}{1296} z_{2}+50 z_{1} z_{2}, \\
& h_{1}=\frac{25}{23328 z_{1}}, \quad h_{2}=-\frac{50}{3} z_{1}, \\
& h_{11}=\frac{5}{36 z_{1} z_{2}}, \quad h_{12}=\frac{5}{108 z_{1} z_{2}}, \quad h_{22}=0 .
\end{aligned}
$$

We will need to know how the propagators transform under the involution. Our guiding principle is the following. We replace the coordinates $z_{i}$ 's with $x_{i}$ 's and all quantities with their transformations in the equations (B.2). We then require that the resulting equations are equivalent to the original ones. Here for the holomorphic ambiguities we can simply replace the $z_{i}$ coordinates with $x_{i}$ 's in the expressions (B.3). Since the Picard-Fuchs operators are invariant under the involution, we argue that the Kahler potential is invariant $\tilde{K}=K$, and the Christoffel symbols transform as

$$
\tilde{\Gamma}_{i j}^{k}=\frac{\partial x_{k}}{\partial z_{l}} \frac{\partial z_{m}}{\partial x_{i}} \frac{\partial z_{n}}{\partial x_{j}} \Gamma_{m n}^{l}+\frac{\partial^{2} z_{m}}{\partial x_{i} \partial x_{j}} \frac{\partial x_{k}}{\partial z_{m}}
$$

We find that it is possible to solve for the transformed propagators $\tilde{S}^{i j}, \tilde{S}^{i}, \tilde{S}$ such that the transformed equations of (B.2) are equivalent to the original ones.

It is easy to check that with the choices of the ambiguities $s_{j k}^{i}$ in (B.3), the transformations are

$$
\tilde{s}_{i j}^{k}=\frac{\partial x_{k}}{\partial z_{l}} \frac{\partial z_{m}}{\partial x_{i}} \frac{\partial z_{n}}{\partial x_{j}} s_{m n}^{l}+\frac{\partial^{2} z_{m}}{\partial x_{i} \partial x_{j}} \frac{\partial x_{k}}{\partial z_{m}} .
$$

The shift exactly cancels that of the Christoffel symbols in the first equation in (B.2), therefore if $S^{i j}$ transform as tensor with a minus sign, the transformed equation is equivalent to the original one by a coordinate transformation.

To solve for the shifts in the transformed propagators $\tilde{S}^{i}$, we combine the involution transformation with the coordinate transformation of the second equation in (B.2). We find the shift in the gauge $s_{i j}^{k}$ exactly cancels the one from the derivative of $S^{j k}$ propagator and all propagators cancel out, so the shifts $f^{i}(i=1,2)$ are determined by the equations

$$
\delta_{i}^{j} f^{k}+\delta_{i}^{k} f^{j}+\tilde{h}_{i}^{j k}+\frac{\partial x_{j}}{\partial z_{l}} \frac{\partial x_{k}}{\partial z_{m}} \frac{\partial z_{n}}{\partial x_{i}} h_{n}^{l m}=0 .
$$


We see that if $h_{i}^{j k}$ transformed as a tensor with a minus sign, the shifts would have vanished. The shifts are results of the non-tensorial transformation of the gauge choice $h_{i}^{j k}$.

Similarly we combine the involution transformation with the coordinate transformation of the third equation in (B.2). Utilizing the solution (3.33), we again find all dependence on propagators cancel, and the shift $f^{0}$ is determined by

$$
\frac{\partial z_{k}}{\partial x_{i}}\left(\partial_{k} f^{j}\right)=2 \delta_{i}^{j} f^{0}-\tilde{s}_{i m}^{j} f^{m}+\tilde{h}_{i}^{j}+\frac{\partial x_{j}}{\partial z_{l}} \frac{\partial z_{n}}{\partial x_{i}} h_{n}^{l}
$$

and we find the the last equation in (3.33)

With the solutions for the shifts (3.33), one can further check that the involution transformation of the fourth and fifth equations in (B.2) are equivalent to the original ones by coordinate transformations.

It is possible to find a gauge choice different from (B.3), such that the shifts in propagators $f^{0}, f^{i}$ vanish. We can keep the propagators $S^{i j}$ and ambiguity $s_{j k}^{i}$, and shift the gauge choice for $h_{i}^{j k}$ in (B.3) by

$$
h_{i}^{j k} \rightarrow h_{i}^{j k}+\delta_{i}^{i} h^{k}+\delta_{i}^{k} h^{j}
$$

where as an example we choose

$$
h^{1}=\frac{5 z_{1}}{12}\left(432 z_{1}-1\right), \quad h^{2}=\frac{5 z_{2}}{216}\left(1+54 z_{2}\right) .
$$

One can easily check that after the shifts, the ambiguity $h_{i}^{j k}$ transforms as a tensor with a minus sign under the involution transformation. So according to (B.6) this new gauge choice will eliminate the shifts $f^{1}, f^{2}$ in the involution transformation of the propagators $S^{i}$. The shift in $h_{i}^{j k}$ will change the propagators $S^{i}, S$ and the other ambiguities $h_{i}^{j}, h_{i}, h_{i j}$ as well, which can be straightforwardly determined.

Similarly we can further shift the ambiguity $h_{i}^{j}$ to cancel the shift $f^{0}$ in the involution transformation of the propagator $S$.

The gauge choice which gives no shifts for the involution transformation of the propagators is conceptually simple, and gives a much better understanding of the involution symmetry as an autoequivalence of the underlying derived categories. However, as it turns out such a gauge choice would introduce pole of $1-432 z_{1}$ in the ambiguities $h_{i}^{j}, h_{i}$. Without detailed calculations, this point can be seen by noting that the shifts (3.33) have poles in $1-432 z_{1}$ which does not appear in the gauge choice in (B.3), so in order to cancel the shifts, we would likely need to introduce poles of $1-432 z_{1}$ in the ambiguities. As a consequence, the holomorphic ambiguities at higher genus would have also poles at $1-432 z_{1}$. The total topological string amplitudes at higher genus should be regular at $1-432 z_{1}$, so the singular part of the holomorphic ambiguities at the pole $1-432 z_{1}$ can be fixed and the gauge choice does not really enlarge the space of holomorphic ambiguities at higher genus but complicates the calculations.

In practical calculations it is simpler to use the gauge (B.3), for which it turns out the holomorphic ambiguities at higher genus have no pole at $1-432 z_{1}$, but only at the conifold divisors $\Delta_{1}$ and $\Delta_{2}$. In the following we will still use the gauge choice (B.3). 


\section{Reducing the ambiguity with the involution symmetry}

We can compute the number of remaining unknown constants for general genus $g$ after imposing the condition (3.5) more precisely. First we can find a particular holomorphic ambiguity such that the total amplitude with the propagator dependent part satisfies the constraint (3.5). Then there is the freedom to add an additional piece $f^{(g)}$ to the holomorphic ambiguity which satisfies the same symmetry condition

$$
\tilde{f}^{(g)}=(-1)^{g-1} f^{(g)} .
$$

The space of such $f^{(g)}$ form a linear space. The number of remaining unknown constants is the dimension of this linear space.

We can define the following linear vector spaces of holomorphic ambiguities

$$
\begin{aligned}
V_{0}^{(g, m, n)} & :=\left\{f \mid f=\frac{p\left(z_{1}\right) z_{2}^{n}}{\left(\Delta_{1} \Delta_{2}\right)^{2 g-2}}, \text { where } p\left(z_{1}\right) \text { is a polynomial degree } m \text { in } z_{1}\right\} . \\
V_{1}^{(g, m, n)} & :=\left\{f \mid f \in V_{0}^{(g, m, n)} \text { and } \tilde{f} \in V_{0}^{(g, m, n)}\right\} . \\
V_{2}^{(g, m, n)} & :=\left\{f \mid f \in V_{1}^{(g, m, n)} \text { and } \tilde{f}=(-1)^{g-1} f\right\} . \\
V_{3}^{(g, m, n)} & :=\left\{f \mid f \in V_{1}^{(g, m, n)} \text { and } \tilde{f}=(-1)^{g} f\right\} .
\end{aligned}
$$

It is easy to check they are indeed linear vector spaces, i.e., any linear combinations of elements of the space is also an element in the space. We would like to construct the explicit linear basis for these spaces.

Clearly $\operatorname{dim}\left(V_{0}^{(g, m, n)}\right)=m+1$. A convenient linearly independent basis is

$$
f\left(z_{1}, z_{2}\right)=\frac{z_{1}^{k}\left(\frac{1}{432}-z_{1}\right)^{m-k} z_{2}^{n}}{\left(\Delta_{1} \Delta_{2}\right)^{2 g-2}}, \quad \text { where } k=0,1, \cdots, m
$$

We can work out the involution transformation

$$
\frac{z_{1}^{k}\left(\frac{1}{432}-z_{1}\right)^{m-k} z_{2}^{n}}{\left(\Delta_{1} \Delta_{2}\right)^{2 g-2}} \rightarrow \frac{(-1)^{n} z_{2}^{n}}{\left(\Delta_{1} \Delta_{2}\right)^{2 g-2}} z_{1}^{m-k+3 n-6 g+6}\left(\frac{1}{432}-z_{1}\right)^{k-3 n+6 g-6} .
$$

We discuss two cases

1. The case $n \geq 2 g-2$. The holomorphic ambiguity in (C.3) $f \in V_{1}^{(g, m, n)}$ for $k \geq$ $3 n-(6 g-6)$. On the other hand, any linear combinations of the basis with $k<$ $3 n-(6 g-6)$ in (C.3) is not in the space $V_{1}^{(g, m, n)}$ since there will be poles of $\left(\frac{1}{432}-z_{1}\right)$ in the numerator. Therefore the space $V_{1}^{(g, m, n)}$ is generated by the linear combinations of $\frac{z_{1}^{k}\left(\frac{1}{432}-z_{1}\right)^{m-k} z_{2}^{n}}{\left(\Delta_{1} \Delta_{2}\right)^{2 g-2}}$, where $k=3 n-(6 g-6), \cdots, m$. In this case the dimension of $V_{1}^{(g, m, n)}$ is

$$
\operatorname{dim}\left(V_{1}^{(g, m, n)}\right)=\left\{\begin{array}{lll}
m+6 g-6-3 n+1, & \text { if } & m+6 g-6-3 n \geq 0 \\
0, & \text { if } & m<3 n-(6 g-6) .
\end{array}\right.
$$


2. The case $n<2 g-2$. A similar discussion shows that the space $V_{1}^{(g, m, n)}$ is generated by the linear combinations of $\frac{z_{1}^{k}\left(\frac{1}{432}-z_{1}\right)^{m-k} z_{2}^{n}}{\left(\Delta_{1} \Delta_{2}\right)^{2 g-2}}$, where $k=0,1, \cdots, m+3 n-6 g+6$. In this case the dimension of $V_{1}^{(g, m, n)}$ is

$$
\operatorname{dim}\left(V_{1}^{(g, m, n)}\right)= \begin{cases}m+3 n-6 g+6+1, & \text { if } \quad m+3 n-6 g+6 \geq 0 \\ 0, & \text { if } \quad m<3 n-(6 g-6) .\end{cases}
$$

In both cases we can write the formula

$$
\operatorname{dim}\left(V_{1}^{(g, m, n)}\right)=\left\{\begin{array}{lll}
m-|3 n-6 g+6|+1, & \text { if } & m \geq|3 n-6 g+6| \\
0, & \text { if } & m<|3 n-6 g+6|
\end{array}\right.
$$

For any element $f \in V_{1}^{(g, m, n)}$ we can write

$$
f=\frac{1}{2}\left(f+(-1)^{g-1} \tilde{f}\right)+\frac{1}{2}\left(f+(-1)^{g} \tilde{f}\right) .
$$

Clearly the first term $\frac{1}{2}\left(f+(-1)^{g-1} \tilde{f}\right) \in V_{2}^{(g, m, n)}$ and the second term $\frac{1}{2}\left(f+(-1)^{g} \tilde{f}\right) \in$ $V_{3}^{(g, m, n)}$. We also note that $V_{2}^{(g, m, n)} \cap V_{3}^{(g, m, n)}=\{0\}$. So we have the decomposition

$$
V_{1}^{(g, m, n)}=V_{2}^{(g, m, n)} \oplus V_{3}^{(g, m, n)}
$$

To construct the linear basis for $V_{2}^{(g, m, n)}$ and $V_{3}^{(g, m, n)}$, we further define two spaces $V_{+}^{(g, m, n)}$ and $V_{-}^{(g, m, n)}$ generated by linearly independent basis

$$
\begin{aligned}
& V_{+}^{(g, m, n)}:=\left\{\text { linear space generated by } \frac{z_{1}^{k_{1}}\left(\frac{1}{432}-z_{1}\right)^{k_{2}} z_{2}^{n}}{\left(\Delta_{1} \Delta_{2}\right)^{2 g-2}} \in V_{0}^{(g, m, n)},\right. \\
& \text { with } k_{1}-k_{2}=3 n-6 g+6 \text {. } \\
& V_{-}^{(g, m, n)}:=\left\{\text { linear space generated by } \frac{\left(\frac{1}{432}-2 z_{1}\right) z_{1}^{k_{1}}\left(\frac{1}{432}-z_{1}\right)^{k_{2}} z_{2}^{n}}{\left(\Delta_{1} \Delta_{2}\right)^{2 g-2}} \in V_{0}^{(g, m, n)},\right. \\
& \text { with } k_{1}-k_{2}=3 n-6 g+6 \text {. }
\end{aligned}
$$

From the transformation (C.4), we can check that for $f \in V_{+}^{(g, m, n)}$ we have $\tilde{f}=(-1)^{n} f$, while for $f \in V_{-}^{(g, m, n)}$ we have $\tilde{f}=(-1)^{n+1} f$. Therefore if $n+g$ is an odd integer, we have $V_{+}^{(g, m, n)} \subseteq V_{2}^{(g, m, n)}$ and $V_{-}^{(g, m, n)} \subseteq V_{3}^{(g, m, n)}$, while if $n+g$ is an even integer, we have $V_{+}^{(g, m, n)} \subseteq V_{3}^{(g, m, n)}$ and $V_{-}^{(g, m, n)} \subseteq V_{2}^{(g, m, n)}$. So we find

$$
\operatorname{dim}\left(V_{+}^{(g, m, n)}\right)+\operatorname{dim}\left(V_{-}^{(g, m, n)}\right) \leq \operatorname{dim}\left(V_{2}^{(g, m, n)}\right)+\operatorname{dim}\left(V_{3}^{(g, m, n)}\right)=\operatorname{dim}\left(V_{1}^{(g, m, n)}\right) .
$$

We can compute the dimensions of $V_{+}^{(g, m, n)}$ and $V_{-}^{(g, m, n)}$ easily from the explicit linearly independent basis in (C.10) and (C.11). Similar to the case of $V_{1}^{(g, m, n)}$, one also need to 
consider two cases $n \geq 2 g-2$ and $n<2 g-2$, and find a universal formulae

$$
\begin{aligned}
& \operatorname{dim}\left(V_{+}^{(g, m, n)}\right)=\left\{\begin{array}{lll}
{\left[\frac{m-|3 n-6 g+6|}{2}\right]+1,} & \text { if } & m \geq|3 n-6 g+6| ; \\
0, & \text { if } & m<|3 n-6 g+6| .
\end{array}\right. \\
& \operatorname{dim}\left(V_{-}^{(g, m, n)}\right)=\left\{\begin{array}{lll}
{\left[\frac{m-1-|3 n-6 g+6|}{2}\right]+1,} & \text { if } & m-1 \geq|3 n-6 g+6| \\
0, & \text { if } & m-1<|3 n-6 g+6| .
\end{array}\right.
\end{aligned}
$$

Comparing with (C.7) we find actually the inequality in (C.12) is saturated. So the spaces $V_{+}^{(g, m, n)}$ and $V_{-}^{(g, m, n)}$ provide the complete linear basis, we have $V_{+}^{(g, m, n)}=$ $V_{2}^{(g, m, n)}, V_{-}^{(g, m, n)}=V_{3}^{(g, m, n)}$ for the case of $n+g$ an odd integer, and $V_{+}^{(g, m, n)}=$ $V_{3}^{(g, m, n)}, V_{-}^{(g, m, n)}=V_{2}^{(g, m, n)}$ for the case of $n+g$ an even integer.

We can estimate the number of unknown constants for the holomorphic ambiguity at large genus $g$ after imposing the involution symmetry, assuming the degree of $z_{1}$ is $7(g-1)$ in the numerator

$$
\sum_{n=0}^{\infty} \operatorname{dim}\left(V_{2}^{(g, 7(g-1), n)}\right)=\sum_{n=0}^{\frac{13(g-1)}{3}} \frac{7(g-1)-|3 n-6(g-1)|}{2} \sim \frac{97}{12} g^{2}, \quad \text { for large } g .
$$

This is about one quarter of the $(7 g-6)(5 g-4)$ unknown constants in the naive ansatz at large genus.

\section{Fibre modularity versus involution symmetry}

In this appendix we will prove that the constraints imposed on the amplitudes and especially on the holomorphic ambiguity from the involution symmetry is equivalent to the constraints from the modularity in the fibre direction discussed in section 4 . In addition the appendix provides many identities that are useful for further discussion of the model [39].

From the solutions ${ }^{32}$ of the (2.10) around the large volume point $\left(z_{1}, z_{2}\right) \sim(0,0)$,

$$
\begin{aligned}
& X_{0}=1+60 z_{1}+13860 z_{1}^{2}+\cdots, \\
& X_{1}=X_{0} \log \left(z_{1}\right)+312 z_{1}+2 z_{2}+\cdots, \\
& X_{2}=X_{0} \log \left(z_{2}\right)+180 z_{1}-6 z_{2}+\cdots,
\end{aligned}
$$

we find

$$
\begin{aligned}
& q_{E}:=q_{1}=\exp \left(\frac{X_{1}}{X_{0}}\right)=z_{1}+312 z_{1}^{2}+2 z_{1} z_{2}+\cdots, \\
& q_{B}:=q_{2}=\exp \left(\frac{X_{2}}{X_{0}}\right)=z_{2}+180 z_{1} z_{2}-6 z_{2}^{2}+\cdots .
\end{aligned}
$$

The above relations can be readily inverted and we can write $z_{1,2}$ as power series of $q_{1,2}$.

\footnotetext{
${ }^{32}$ We lower the index on the A-periods $X^{i}$ in order to avoid cluttering with exponentials.
} 
If we keep the gauge $X_{0}^{2 g-2}$ explicit and note that $q_{B}$ is defined without the shift (4.1) then (4.4), (4.5) read

$$
X_{0}^{2 g-2} \mathcal{F}^{(g)}=\sum_{k=0}^{\infty} P_{k}^{(g)}\left(q_{E}\right)\left(\frac{q_{E}}{\eta\left(q_{E}\right)^{24}}\right)^{\frac{3 k}{2}} q_{B}^{k}, \quad g \geq 1,
$$

while for the case of $g=0$ we do not need the factor of $X_{0}^{-2}$ in the conventional definition of the prepotential.

From section 4 it follows for the $X_{18}(1,1,1,6,9)$ model that the $P_{k}^{(g)}\left(q_{E}\right)$ are quasimodular forms of $\mathrm{SL}(2, \mathbb{Z})$, of modular weight $18 k+2 g-2$ and satisfy (4.13) as a specialization of (4.8). We would like to understand whether and to what extent this constraint from the modularity of the fiber fixes the holomorphic ambiguity at higher genus. Suppose at genus $g \geq 2$ we have found a particular holomorphic ambiguity such that the topological string amplitude $\mathcal{F}^{(g)}$ satisfies the fiber modularity constrain. Then we may add an additional holomorphic ambiguity $f^{(g)}$, whose expansion is

$$
X_{0}^{2 g-2} f^{(g)}=\sum_{k=0}^{\infty} p_{k}^{(g)}\left(q_{E}\right)\left(\frac{q_{E}}{\eta\left(q_{E}\right)^{24}}\right)^{\frac{3 k}{2}} q_{B}^{k},
$$

we require $p_{k}^{(g)}\left(q_{E}\right)$ to be also a quasi-modular form of weight $18 k+2 g-2$. Furthermore, since the sum $P_{k}^{(g)}\left(q_{E}\right)+p_{k}^{(g)}\left(q_{E}\right)$ also need to satisfy the equation (4.13), we find

$$
\frac{\partial p_{k}^{(g)}}{\partial E_{2}}=-\frac{1}{12} \sum_{s=1}^{k-1} s(k-s) p_{s}^{(g)} P_{k-s}^{(0)} .
$$

In particular for the case $k=0,1$, this equation is understood as $\frac{\partial p_{k}^{(g)}}{\partial E_{2}}=0$, i.e. $p_{0}^{(g)}$ and $p_{1}^{(g)}$ are always modular forms.

It turns out that not all rational functions $f^{(g)}$ satisfy the fiber modularity constraint (D.4), (D.5), so the fiber modularity does impose some conditions on holomorphic ambiguities. However we find that there exist some non-vanishing rational functions $f^{(g)}$ satisfying these conditions, so the fiber modularity does not completely fix the holomorphic ambiguity. We see that the BCOV holomorphic anomaly equation alone does not implies the modularity of the fiber at higher genus.

However it turns out that the modularity of the fiber is equivalent to the involution symmetry. It gives more precise bounds for the degrees in the polynomials in the numerators of the holomorphic ambiguity and further reduces the number of unknown constants in the holomorphic ambiguity. To understand this equivalence, we consider a holomorphic ambiguity $f \in V_{0}^{(g,+\infty, n)}$. Since $f \sim z_{2}^{n} \sim q_{B}^{n}$ in the small $z$ limit, it is easy to see that the first non-vanish coefficient in the expansion in base degree (D.4) is that of $q_{B}^{n}$

$$
X_{0}^{2 g-2} f=\sum_{k=n}^{\infty} p_{k}\left(q_{E}\right)\left(\frac{q_{E}}{\eta\left(q_{E}\right)^{24}}\right)^{\frac{3 k}{2}} q_{B}^{k},
$$

According to (D.5) the first coefficient $p_{n}\left(q_{E}\right)$ has no $E_{2}$ dependence and is purely a modular form, i.e. polynomials of $E_{4}$ and $E_{6}$. 
We define the linear space

$$
W_{0}^{(g, n)}:=\left\{f \mid f \in V_{0}^{(g,+\infty, n)} \text {, and the first non-vanishing coefficient } p_{n}\left(q_{E}\right)\right.
$$

in the expansion (D.6) is a $\mathrm{SL}(2, \mathbb{Z})$ modular form of weight $18 n+2 g-2\}$.

We will prove the following proposition

\section{Proposition 3.}

$$
W_{0}^{(g, n)}=V_{2}^{\left(g,\left[\frac{19}{3}(g-1)\right], n\right)}
$$

Proof. The proof will proceed in two steps. We shall first show $V_{2}^{\left(g,\left[\frac{19}{3}(g-1)\right], n\right)} \subseteq W_{0}^{(g, n)}$, and then $\operatorname{dim}\left(V_{2}^{\left(g,\left[\frac{19}{3}(g-1)\right], n\right)}\right) \geq \operatorname{dim}\left(W_{0}^{(g, n)}\right)$. We will see how the number $\left[\frac{19}{3}(g-1)\right]$ appears in the process.

In order to prove the Proposition 3, we only need the leading order term in the small $z_{2}$ expansion. The first Picard-Fuchs equation in (2.10) in the $z_{2} \rightarrow 0$ limit has been studied before in $[34,54]$. We find the power series solution and A-model parameter $q_{E}$ are determined by the exact relations

$$
E_{4}\left(q_{E}\right)=X_{0}^{4}, \quad z_{1}\left(1-432 z_{1}\right)=\frac{\eta\left(q_{E}\right)^{24}}{E_{4}\left(q_{E}\right)^{3}},
$$

where we have taken the $z_{2} \rightarrow 0$ limit for $X_{0}$ and $q_{E}$. We will drop the argument $q_{E}=q_{1}$ in the modular forms and $\eta$ function for simplicity.

For the other logarithmic solution we need to keep the log term in $z_{2} \rightarrow 0$ limit

$$
X_{2}=X_{0} \log \left(z_{2}\right)+\xi\left(z_{1}\right)+\mathcal{O}\left(z_{2}\right)
$$

where $\xi\left(z_{1}\right)=180 z_{1}+\mathcal{O}\left(z_{1}^{2}\right)$ is a power series. Using the first Picard-Fuchs equation in $(2.10)$ we find

$$
\left[\theta_{1}^{2}-12 z_{1}\left(6 \theta_{1}+1\right)\left(6 \theta_{1}+5\right)\right] \xi\left(z_{1}\right)=3 \theta_{1} X_{0}
$$

There is an exact solution for $\xi\left(z_{1}\right)$ using (D.8) and the well-known Ramanujan derivative relations for quasi-modular forms

$$
\xi\left(z_{1}\right)=-\frac{3}{2} X_{0} \log \left[\frac{q_{1}\left(1-432 z_{1}\right)}{z_{1}}\right] .
$$

Of course one can add any linear combination of two solutions of PF equation in the $z_{2} \rightarrow 0$ limit, which are $E_{4}\left(q_{1}\right)^{\frac{1}{4}}$ and $\log \left(q_{1}\right) E_{4}\left(q_{1}\right)^{\frac{1}{4}}$. The above solution is the only one with the correct asymptotic behavior $\xi\left(z_{1}\right) \sim z_{1}$ for small $z_{1}$. So the A-model parameter $q_{B}$ is

$$
q_{B}=z_{2} \exp \left(\frac{\xi\left(z_{1}\right)}{X_{0}}\right)=z_{2}\left(\frac{q_{1}\left(1-432 z_{1}\right)}{z_{1}}\right)^{-\frac{3}{2}}+\mathcal{O}\left(z_{2}^{2}\right)
$$

We first discuss the case that $n+g$ is an odd integer. Suppose $f \in V_{2}^{\left(g,\left[\frac{19}{3}(g-1)\right], n\right)}=$ $V_{+}^{\left(g,\left[\frac{19}{3}(g-1)\right], n\right)}$ is a base vector in (C.10)

$$
\begin{aligned}
& f=\frac{z_{1}^{k_{1}}\left(\frac{1}{432}-z_{1}\right)^{k_{2}} z_{2}^{n}}{\left(\Delta_{1} \Delta_{2}\right)^{2 g-2}}, \\
& \text { with } k_{1} \geq 0, k_{2} \geq 0, k_{1}+k_{2} \leq \frac{19}{3}(g-1), k_{1}-k_{2}=3 n-6 g+6,
\end{aligned}
$$


then the leading term in the expansion (D.6) is

$$
\begin{aligned}
X_{0}^{2 g-2} f & \sim E_{4}^{\frac{g-1}{2}} z_{1}^{k_{1}-\frac{3 n}{2}}\left(1-432 z_{1}\right)^{k_{2}+\frac{3 n}{2}-6 g+6} \eta^{36 n}\left(\frac{q_{E}}{\eta^{24}}\right)^{\frac{3 n}{2}} q_{B}^{n} \\
& \sim E_{4}^{\frac{g-1}{2}+\frac{9 n}{2}-3 k_{1}} \eta^{24 k_{1}}\left(\frac{q_{E}}{\eta^{24}}\right)^{\frac{3 n}{2}} q_{B}^{n},
\end{aligned}
$$

where we have used the relations (D.8) and ignore constant factors. Since $n+g$ is an odd integer, the exponent of $E_{4}$ is an integer. Furthermore

$$
\frac{g-1}{2}+\frac{9 n}{2}-3 k_{1}=\frac{19}{2}(g-1)-\frac{3}{2}\left(k_{1}+k_{2}\right) \geq 0,
$$

so the coefficient $E_{4}^{\frac{g-1}{2}+\frac{9 n}{2}-3 k_{1}} \eta^{24 k_{1}}$ is a modular form of weight $18 n+2 g-2$.

The case of $n+g$ an even integer is similar. We need to use the formula $1-864 z_{1}=$ $E_{6} / E_{4}^{\frac{3}{2}}$. In both cases we find $V_{2}^{\left(g,\left[\frac{19}{3}(g-1)\right], n\right)} \subseteq W_{0}^{(g, n)}$.

Now we compute $\operatorname{dim}\left(W_{0}^{(g, n)}\right)$. We discuss two cases.

1. The case of $n \leq 2 g-2$. We consider the dimension of the space $\mathcal{M}_{18 n+2 g-2}$ of modular forms of weight $18 n+2 g-2$. It is clear $\operatorname{dim}\left(\mathcal{M}_{18 n+2 g-2}\right)=\left[\frac{9 n+g-1}{6}\right]+1$ if $n+g$ is odd, and $\operatorname{dim}\left(\mathcal{M}_{18 n+2 g-2}\right)=\left[\frac{9 n+g-4}{6}\right]+1$ if $n+g$ is even. This is exactly the dimension $\operatorname{dim}\left(V_{2}^{\left(g,\left[\frac{19}{3}(g-1)\right], n\right)}\right)$ according to (C.13). The expansion (D.6) is a linear map from $W_{0}^{(g, n)}$ to $\mathcal{M}_{18 n+2 g-2}$ and the kernel is the zero element. So in this case we find

$$
\operatorname{dim}\left(W_{0}^{(g, n)}\right) \leq \operatorname{dim}\left(\mathcal{M}_{18 n+2 g-2}\right)=\operatorname{dim}\left(V_{2}^{\left(g,\left[\frac{19}{3}(g-1)\right], n\right)}\right)
$$

2. The case of $n>2 g-2$. Suppose $f=\frac{h\left(z_{1}\right) z_{2}^{n}}{\left(\Delta_{1} \Delta_{2}\right)^{2 g-2}}$ where $h\left(z_{1}\right)$ is a polynomial of $z_{1}$. We find the leading term in the expansion (D.6)

$$
X_{0}^{2 g-2} f \sim E_{4}^{\frac{g-1}{2}} \frac{h\left(z_{1}\right)}{z_{1}^{\frac{3 n}{2}}\left(1-432 z_{1}\right)^{6 g-6-\frac{3 n}{2}}} \eta^{36 n}\left(\frac{q_{E}}{\eta^{24}}\right)^{\frac{3 n}{2}} q_{B}^{n},
$$

We consider the zero at $1-432 z_{1} \sim 0$, which correspond to $E_{6}+E_{4}^{\frac{3}{2}} \sim 0$. From see $(\mathrm{D} .8)$ we see $\eta^{24} \sim\left(1-432 z_{1}\right)$. So the leading coefficient in (D.6) $p_{n}\left(q_{E}\right) \sim$ $\left(1-432 z_{1}\right)^{3 n-6 g+6}$. If $p_{n}\left(q_{E}\right)$ is a modular form of weight $18 n+2 g-2$, then $\frac{p_{n}\left(q_{E}\right)}{\eta^{72(n-2 g+2)}}$ should be also a modular form of weight $74(g-1)-18 n$. So we find

$$
\operatorname{dim}\left(W_{0}^{(g, n)}\right) \leq \operatorname{dim}\left(\mathcal{M}_{74(g-1)-18 n}\right)=\operatorname{dim}\left(V_{2}^{\left(g,\left[\frac{19}{3}(g-1)\right], n\right)}\right)
$$

So we have proven $W_{0}^{(g, n)}=V_{2}^{\left(g,\left[\frac{19}{3}(g-1)\right], n\right)}$, and in the proof we see that they are also isomorphic to the space of modular forms $\mathcal{M}_{18 n+2 g-2}$ for the $n \leq 2 g-2$ case, and $\mathcal{M}_{74(g-1)-18 n}$ for the $n>2 g-2$ case. We further conjecture this is sufficient for the full fiber modularity constrain of the higher terms. 
Conjecture 1. For $f \in W_{0}^{(g, n)}$ with the expansion (D.6), the higher order terms $p_{k}\left(q_{E}\right)$ with $k>n$ are quasi-modular forms of weight $18 k+2 g-2$ and satisfy the equation (D.5).

If our conjecture is correct, then the space of holomorphic ambiguities at genus $g$ satisfying the modularity constraint of the elliptic fiber is linearly generated by $\sum_{n=0}^{\infty} \oplus W_{0}^{(g, n)}=$ $\sum_{n=0}^{\infty} \oplus V_{2}^{\left(g,\left[\frac{19}{3}(g-1)\right], n\right)}$. For example, the explicit basis at genus $g=2$ are the the following 7 elements

$$
\begin{aligned}
& \frac{\left(1-432 z_{1}\right)^{3} z_{2}}{\left(\Delta_{1} \Delta_{2}\right)^{2}}, \quad \frac{\left(1-432 z_{1}\right)^{4} z_{1} z_{2}}{\left(\Delta_{1} \Delta_{2}\right)^{2}}, \quad \frac{\left(1-864 z_{1}\right) z_{2}^{2}}{\left(\Delta_{1} \Delta_{2}\right)^{2}}, \quad \frac{\left(1-864 z_{1}\right)\left(1-432 z_{1}\right) z_{1} z_{2}^{2}}{\left(\Delta_{1} \Delta_{2}\right)^{2}}, \\
& \frac{\left(1-864 z_{1}\right)\left(1-432 z_{1}\right)^{2} z_{1}^{2} z_{2}^{2}}{\left(\Delta_{1} \Delta_{2}\right)^{2}}, \quad \frac{z_{1}^{3} z_{2}^{3}}{\left(\Delta_{1} \Delta_{2}\right)^{2}}, \quad \frac{\left(1-432 z_{1}\right) z_{1}^{4} z_{2}^{3}}{\left(\Delta_{1} \Delta_{2}\right)^{2}} .
\end{aligned}
$$

The numbers of unknown constants at genus 2, 3, 4, 5, 6 after using the fiber modularity constrain are 7, 31, 70, 109, 176 respectively.

We can compute the number of unknown constants in the holomorphic ambiguity at genus $g$ with the formula (C.13), and we only need to change the bound for degree of $z_{1}$ from $7(g-1)$ to $\frac{19}{3}(g-1)$ in the calculations (C.14)

$$
\sum_{n=0}^{\infty} \operatorname{dim}\left(V_{2}^{\left(g, \frac{19}{3}(g-1), n\right)}\right)=\sum_{n=0}^{\frac{37}{9}(g-1)} \frac{\frac{19}{3}(g-1)-|3 n-6(g-1)|}{2} \sim \frac{721}{108} g^{2}, \text { for large } g,
$$

which is a better estimate than (C.14).

The degree $\left[\frac{19}{3}(g-1)\right]$ of $z_{1}$ from fiber modularity (3) can be also derived from the regularity condition at $z_{1} \sim \infty$. We can use the local coordinates $\left(\frac{1}{z_{1}}, z_{2}\right)$ around the point $\left(z_{1}, z_{2}\right)=(\infty, 0)$. It is easy to check that the power series solutions to the PF equations have the asymptotic behavior $w \sim z_{1}^{-\frac{1}{6}}$ or $w \sim z_{1}^{-\frac{5}{6}}$. For the scaling factor $X_{0}$ in the topological string amplitude we take the one with the lowest exponent $X_{0} \sim z_{1}^{-\frac{1}{6}}$. So the scaling exponent including the conifold divisors is

$$
\left(\frac{X_{0}}{\Delta_{1} \Delta_{2}}\right)^{2 g-2} \sim z_{1}^{-\frac{19}{3}(g-1)}
$$

and the regularity of $X_{0}^{2 g-2} \mathcal{F}^{(g)}$ at $z_{1} \sim \infty$ requires the degree of $z_{1}$ at the numerator in the holomorphic ambiguity be no bigger than $\frac{19}{3}(g-1)$. The non-zero scaling exponent in $X_{0}$ around $z_{1} \sim \infty$ is characteristic of compact Calabi-Yau models, and we will see that it also is responsible for preventing the solution of the model at large genus as in one-parameter models like the quintic.

\section{D.1 Some (quasi-)modularity formulae}

In order to understand Conjecture 1, we provide some more formulae relating the complex structure coordinates $z_{1,2}$ to (quasi)modular forms of the fiber parameter. We note that for $f \in W_{0}^{(g, n)}$ in the case of $n+g$ odd, we can factor (D.13) with $k_{2}=k_{1}-3 n+6 g-6$ as the following

$$
f=\left[z_{1}\left(1-432 z_{1}\right)\right]^{k_{1}}\left(\frac{z_{2}}{\left(1-432 z_{1}\right)^{3}}\right)^{n}\left(\frac{\Delta_{1} \Delta_{2}}{\left(1-432 z_{1}\right)^{3}}\right)^{2-2 g} .
$$


So we need to understand the expansion of $z_{1}\left(1-432 z_{1}\right), \frac{z_{2}}{\left(1-432 z_{1}\right)^{3}}$, and $\frac{\Delta_{1} \Delta_{2}}{\left(1-432 z_{1}\right)^{3}}$ in terms of exponential of flat coordinates $q_{E}, q_{B}$. There is an extra factor of $1-864 z_{1}$ in the case of $n+g$ an even integer. Furthermore, we also need to consider the A-model scaling factor $X_{0}$ which is the power series solution to the Picard-Fuchs equation.

We define the parameter

$$
Z:=\frac{z_{2}}{\left(1-432 z_{1}\right)^{3} E_{4}\left(q_{E}\right)^{\frac{9}{2}}}
$$

and as a first step we expand some components as asymptotic series of $Z$, and we find that the coefficients are modular forms of fiber parameter $q_{E}$ as the followings

$$
\begin{aligned}
& z_{1}\left(1-432 z_{1}\right)=\frac{\eta^{24}}{E_{4}^{3}}\left[1-\frac{77 E_{4}^{3} E_{6}+211 E_{6}^{3}}{144} Z+\frac{1}{165888}\left(-11858 E_{4}^{9}+330395 E_{4}^{6} E_{6}^{2}\right.\right. \\
& \left.\left.+1434560 E_{4}^{3} E_{6}^{4}+1066999 E_{6}^{6}\right) Z^{2}+\mathcal{O}\left(Z^{3}\right)\right], \\
& \frac{\Delta_{1} \Delta_{2}}{\left(1-432 z_{1}\right)^{3}}=1+\frac{27}{4}\left(3 E_{4}^{3} E_{6}+E_{6}^{3}\right) Z-\frac{9}{128}\left(E_{4}^{3}-E_{6}^{2}\right)\left(85 E_{4}^{6}-612 E_{4}^{3} E_{6}^{2}-49 E_{6}^{4}\right) Z^{2} \\
& +\mathcal{O}\left(Z^{3}\right) \\
& 1-864 z_{1}=\frac{1}{E_{4}^{\frac{3}{2}}}\left[E_{6}+\frac{1}{288}\left(E_{4}^{3}-E_{6}^{2}\right)\left(77 E_{4}^{3}+211 E_{6}^{2}\right) Z-\frac{1}{331776} E_{6}\left(E_{4}^{3}-E_{6}^{2}\right)\right. \\
& \left.\cdot\left(383525 E_{4}^{6}+1458614 E_{4}^{3} E_{6}^{2}+977957 E_{6}^{4}\right) Z^{2}+\mathcal{O}\left(Z^{3}\right)\right], \\
& X_{0}=E_{4}^{\frac{1}{4}}\left[1-\frac{5}{72} E_{6}\left(E_{4}^{3}-E_{6}^{2}\right) Z+\frac{5}{7962624}\left(E_{4}^{3}-E_{6}^{2}\right)\right. \\
& \left.\cdot\left(15935 E_{4}^{6}+488258 E_{4}^{3} E_{6}^{2}+435839 E_{6}^{4}\right) Z^{2}+\mathcal{O}\left(Z^{3}\right)\right],
\end{aligned}
$$

Here we fix the modular forms in low orders by perturbative calculations. In general we conjecture the following

Conjecture 2. With the appropriate normalization factors as in (D.24), (D.25), (D.26), (D.27), the coefficients of $Z^{n}$ in the expansion are modular forms, and the modular weights are $18 n$ for the cases of $z_{1}\left(1-432 z_{1}\right), \frac{\Delta_{1} \Delta_{2}}{\left(1-432 z_{1}\right)^{3}}, X_{0}$, and $18 n+6$ for the case of $1-864 z_{1}$.

In the next subsection D.2, we shall prove this modularity conjecture. We note that we only need to prove the modularity of the coefficients of the expansion of $z_{1}\left(1-432 z_{1}\right)$ and $1-864 z_{1}$, which immediately imply that of $\frac{\Delta_{1} \Delta_{2}}{\left(1-432 z_{1}\right)^{3}}$, since we can write

$$
\begin{aligned}
\frac{\Delta_{1} \Delta_{2}}{\left(1-432 z_{1}\right)^{3}}= & 1-\frac{27 z_{2}}{\left(1-432 z_{1}\right)^{3}}\left(1-864 z_{1}\right)\left[\left(1-864 z_{1}\right)^{2}+1296 z_{1}\left(1-432 z_{1}\right)\right] \\
& -\frac{\left(27 z_{2}\right)^{2}}{\left(1-432 z_{1}\right)^{6}}\left[432 z_{1}\left(1-432 z_{1}\right)\right]^{3}
\end{aligned}
$$


We also notice that the higher order modular form coefficients in the expansion formula (D.26) contain a factor of $\eta^{24}=\frac{E_{4}^{3}-E_{6}^{2}}{1728}$. This condition is sufficient to ensure the modularity of expansion (D.24), and therefore that of (D.25) as well. To see this, suppose

$$
1-864 z_{1}=\frac{1}{E_{4}^{\frac{3}{2}}}\left[E_{6}+\left(E_{4}^{3}-E_{6}^{2}\right) \sum_{n=1}^{\infty} f_{18 n-6} Z^{n}\right],
$$

where $f_{18 n-6}$ are some modular forms of weight $18 n-6$. We can compute (D.24) easily

$$
z_{1}\left(1-432 z_{1}\right)=\frac{\eta^{24}}{E_{4}^{3}}\left[1-2 E_{6}\left(\sum_{n=1}^{\infty} f_{18 n-6} Z^{n}\right)+\left(E_{4}^{3}-E_{6}^{2}\right)\left(\sum_{n=1}^{\infty} f_{18 n-6} Z^{n}\right)^{2}\right]
$$

Now it is easy to see that if Conjecture 2 is correct, then for $f \in W_{0}^{(g, n)}$, it has the following expansion

$$
f=\sum_{n=0}^{+\infty} f_{n} Z^{n}
$$

where $f_{n}$ are modular forms of $q_{E}$ of modular weight $18 n+2 g-2$.

The next step is to expand $Z$ parameter in terms of the base coordinate $q_{B}$. For convenience we denote the normalized Kahler coordinate

$$
Q:=\left(\frac{q_{E}}{\eta^{24}}\right)^{\frac{3}{2}} q_{B}
$$

and we find the formulae for low orders

$$
\begin{aligned}
Z=Q+ & \frac{1}{576}\left[-E_{2} E_{4}\left(31 E_{4}^{3}+113 E_{6}^{2}\right)+1759 E_{4}^{3} E_{6}+1841 E_{6}^{3}\right] Q^{2} \\
& +\frac{1}{15925248}\left[72 E_{2}^{2} E_{4}^{2}\left(31 E_{4}^{3}+113 E_{6}^{2}\right)^{2}\right. \\
& \quad-108 E_{2} E_{4} E_{6}\left(25249 E_{4}^{6}+143422 E_{4}^{3} E_{6}^{2}+163105 E_{6}^{4}\right) \\
& \left.\quad+102919 E_{4}^{9}+33568143 E_{4}^{6} E_{6}^{2}+79676877 E_{4}^{3} E_{6}^{4}+64318109 E_{6}^{6}\right] Q^{3}+\mathcal{O}\left(Q^{4}\right)
\end{aligned}
$$

We can check that the coefficients $c_{n}$ of $Q^{n}$ are quasi-modular forms, and satisfy the following equation as in (D.5)

$$
\frac{\partial c_{n}}{\partial E_{2}}=-\frac{1}{12} \sum_{s=1}^{n-1} s(n-s) c_{n-s} P_{s}^{(0)}
$$

where the $P_{n}^{(0)}$ are the quasi-modular forms of the prepotential expanded in terms of base degree, for example

$$
\begin{aligned}
P_{1}^{(0)}= & \frac{E_{4}}{48}\left(31 E_{4}^{3}+113 E_{6}^{2}\right), \\
P_{2}^{(0)}=-\frac{1}{221184}\left[4 E _ { 2 } E _ { 4 } ^ { 2 } \left(31 E_{4}^{3}+\right.\right. & \left.113 E_{6}^{2}\right)^{2}+208991 E_{4}^{7} E_{6} \\
& \left.+755906 E_{4}^{4} E_{6}^{3}+196319 E_{4} E_{6}^{5}\right]
\end{aligned}
$$


We define the following space

$$
\begin{aligned}
W_{1}:=\left\{f \mid f=\sum_{n=1}^{\infty} c_{n} Q^{n}, \text { where } c_{n}\right. \text { are quasi-modular forms } \\
\text { and satisfy equation (D.34) }\} .
\end{aligned}
$$

Here we do not specify the modular weights of $c_{n}$, so unlike the previous cases, the space $W_{1}$ is not a linear space. Rather, we will see it is a graded module over modular forms. Since we know the modular weight of $P_{n}^{(0)}$ is $18 n-2$, the modular weights of all $c_{n}$ 's are actually determined by the first non-vanishing coefficient according to equation (D.34). It is clear if $f \in W_{1}$ and $g$ is a modular form, then $f g \in W_{1}$.

The equation (D.34) can be equivalently written as

$$
\frac{\partial f}{\partial E_{2}}=-\frac{1}{12} Q^{2}\left(\partial_{Q} f\right) \partial_{Q} P^{(0)}
$$

where we define the generating function $P^{(0)}:=\sum_{s=1}^{\infty} P_{s}^{(0)} Q^{s}$. Suppose $f \in W_{1}$ and $g \in W_{1}$, then we have $f g \in W_{1}$ since

$$
\frac{\partial(f g)}{\partial E_{2}}=-\frac{1}{12} g Q^{2}\left(\partial_{Q} f\right) \partial_{Q} P^{(0)}-\frac{1}{12} f Q^{2}\left(\partial_{Q} f\right) \partial_{Q} P^{(0)}=-\frac{1}{12} Q^{2}\left(\partial_{Q} f g\right) \partial_{Q} P^{(0)} .
$$

Furthermore, If $f \in W_{1}$, then any algebraic function of $f$ would also satisfies the equation (D.37). However, $f$ may not have the structure of $Q$-expansion defined by $W_{1}$ space in (D.36), and the coefficients $c_{n}$ may be only algebraic functions of quasi-modular forms.

The equation (D.37) can be further written in a simpler way which will be useful later. In the left hand side of (D.37), we regard $f$ as function of $f\left(Q, E_{2}, E_{4}, E_{6}\right)$ when taking partial derivative with respect to $E_{2}$. Alternatively, we can also use inverse function and regard $Q$ as function of $Q\left(f, E_{2}, E_{4}, E_{6}\right)$. In this perspective we have $\frac{\partial f}{\partial E_{2}}=0$ and $\frac{\partial Q}{\partial E_{2}} \neq 0$. We can take partial derivative of $E_{2}$ on $f=\sum_{n=1}^{\infty} c_{n} Q^{n}$, and find

$$
\sum_{n=1}^{\infty}\left(\partial_{E_{2}} c_{n}\right) Q^{n}+\left(\partial_{Q} f\right)\left(\partial_{E_{2}} Q\right)=0 .
$$

So we can write equation (D.37) as

$$
\frac{\partial \log (Q)}{\partial E_{2}}=\frac{1}{12} Q \partial_{Q} P^{(0)} .
$$

Based on the perturbative calculations (D.33), we conjecture

Conjecture 3. $Z \in W_{1}$.

If our conjecture is correct, then obviously it is also true that $Z^{n} \in W_{1}$ for any positive integer $n$. Using the expansion (D.31) and keeping track of the modular weights, we can see that Conjectures 2 and 3 and calculations in this subsection would imply the validity of the main conjecture, Conjecture 1. 


\section{D.2 Proof of Conjecture 2 and Conjecture 3.}

In this subsection we shall prove the Conjectures 2 and 3 for our compact model. The proof uses some ingredients in the proof of genus zero modular anomaly equation for the non-compact half K3 model in [34, 54], but it is much more complicated for compact model.

To solve the Picard-Fuchs equations (2.10), it is convenient to define the variable $\tilde{q}$, which is related to $z_{1}$ by

$$
J(\tilde{q})=\frac{1}{z_{1}\left(1-432 z_{1}\right)},
$$

with the asymptotic behavior $\tilde{q} \sim z_{1} \sim 0$ in small $z_{1}$ limit. This is the Kahler parameter in local large base limit $z_{2} \rightarrow 0$, but in the compact model, it is different from the physical Kähler parameter $q_{1}$ which depends on both $z_{1}$ and $z_{2}$. To be clear in this subsection we will keep the arguments $\tilde{q}$ and $q_{1}$ in the modular functions, and we also define the logarithmic flat coordinates $t_{1}=\log \left(q_{1}\right)$ and $\tilde{t}=\log (\tilde{q})$.

We can write the ansatz for the solution to the equations (2.10) in small $z_{2}$ power series expansion

$$
w=\sum_{n=0}^{\infty} c_{n}\left(z_{1}\right) z_{2}^{n}
$$

It is well known in the local $z_{2} \rightarrow 0$ limit, there are two linearly independent solutions, which can be written as $E_{4}(\tilde{q})^{\frac{1}{4}}$ and $\log (\tilde{q}) E_{4}(\tilde{q})^{\frac{1}{4}}$, in terms of the modular parameter $\tilde{q}$ defined in (D.41). So the initial function $c_{0}\left(z_{1}\right)$ in the expansion is $E_{4}(\tilde{q})^{\frac{1}{4}}$ or $\log (\tilde{q}) E_{4}(\tilde{q})^{\frac{1}{4}}$.

The first and second PF equations in (2.10) become

$$
\begin{aligned}
12 z_{1}\left(6 \theta_{1}+1\right)\left(6 \theta_{1}+5\right) c_{n}\left(z_{1}\right) & =\theta_{1}\left(\theta_{1}-3 n\right) c_{n}\left(z_{1}\right), \\
c_{n+1}\left(z_{1}\right) & =\frac{1}{(n+1)^{3}} \prod_{i=0}^{2}\left(\theta_{1}-3 n-i\right) c_{n}\left(z_{1}\right)
\end{aligned}
$$

where $\theta_{z_{1}}=z_{1} \partial_{z_{1}}$. We see that the second equation provides a recursion relation to compute the higher order coefficients $c_{n}\left(z_{1}\right)$. Furthermore, one can check that the two equations are consistent, i.e. the $c_{n+1}$ from recursion in the second equation also satisfies the first equation for $c_{n}$ with by replacing $n$ with $n+1$. The recursion implies

$$
c_{n}\left(z_{1}\right)=\frac{1}{(n !)^{3}} \prod_{k=0}^{3 n-1}\left(\theta_{z_{1}}-k\right) c_{0}\left(z_{1}\right)
$$

A particular modularity structure for such recursion was derived in [34, 54]. Here we repeat the analysis. From the relation (D.41) it is easy to compute

$$
1-864 z_{1}=\frac{E_{6}(\tilde{q})}{E_{4}(\tilde{q})^{\frac{3}{2}}}, \quad \theta_{z_{1}}=\frac{2 E_{4}(\tilde{q})}{E_{4}(\tilde{q})^{\frac{3}{2}}+E_{6}(\tilde{q})} \partial_{\tilde{t}} .
$$


We can write some derivative formulae

$$
\begin{aligned}
\partial_{\tilde{t}} \frac{E_{4}(\tilde{q})^{\frac{3}{2}}}{E_{4}(\tilde{q})^{\frac{3}{2}}+E_{6}(\tilde{q})} & =\frac{E_{4}(\tilde{q})^{\frac{1}{2}}}{2} \frac{E_{4}(\tilde{q})^{\frac{3}{2}}-E_{6}(\tilde{q})}{E_{4}(\tilde{q})^{\frac{3}{2}}+E_{6}(\tilde{q})} \\
\partial_{\tilde{t}} \frac{E_{2}(\tilde{q})}{E_{4}(\tilde{q})^{\frac{1}{2}}} & =-\frac{E_{2}(\tilde{q})^{2} E_{4}(\tilde{q})-2 E_{2}(\tilde{q}) E_{6}(\tilde{q})+E_{4}(\tilde{q})^{2}}{12 E_{4}(\tilde{q})^{\frac{3}{2}}} \\
\partial_{\tilde{t}} \frac{E_{6}(\tilde{q})}{E_{4}(\tilde{q})^{\frac{3}{2}}} & =-\frac{E_{4}(\tilde{q})^{3}-E_{6}(\tilde{q})^{2}}{2 E_{4}(\tilde{q})^{\frac{5}{2}}} \\
\partial_{\tilde{t}} E_{4}(\tilde{q})^{\frac{1}{4}} & =\frac{E_{2}(\tilde{q}) E_{4}(\tilde{q})-E_{6}(\tilde{q})}{12 E_{4}(\tilde{q})^{\frac{3}{4}}} .
\end{aligned}
$$

First we consider the case $c_{0}\left(z_{1}\right)=E_{4}(\tilde{q})^{\frac{1}{4}}$. From the derivative formulae (D.46) one can deduce inductively that the recursion function

$$
\prod_{k=0}^{n-1}\left(\theta_{z_{1}}-k\right) E_{4}(\tilde{q})^{\frac{1}{4}} \sim \frac{E_{4}(\tilde{q})^{\frac{1}{4}}}{\left(E_{4}(\tilde{q})^{\frac{3}{2}}+E_{6}(\tilde{q})\right)^{n}} P_{6 n}\left(E_{2}, E_{4}, E_{6}\right),
$$

where $P_{6 n}\left(E_{2}, E_{4}, E_{6}\right)$ is a quasi-modular form of weight $6 n$ and is linear in $E_{2}(\tilde{q})$. We can write

$$
\prod_{k=0}^{n-1}\left(\theta_{z_{1}}-k\right) E_{4}(\tilde{q})^{\frac{1}{4}}=\frac{E_{4}(\tilde{q})^{\frac{1}{4}}}{\left(E_{4}(\tilde{q})^{\frac{3}{2}}+E_{6}(\tilde{q})\right)^{n}}\left(a_{n} E_{2}(\tilde{q})+b_{n}\right),
$$

where $a_{n}$ and $b_{n}$ are modular forms of weight $6 n-2$ and $6 n$, without $E_{2}$ dependence. Some low order formulae are the followings

$$
\begin{aligned}
a_{0} & =0, \quad b_{0}=1, & a_{1} & =\frac{E_{4}(\tilde{q})}{6}, \\
a_{2} & =-\frac{E_{4}(\tilde{q}) E_{6}(\tilde{q})}{3}, & b_{2} & =\frac{5 E_{4}(\tilde{q})^{3}+7 E_{6}(\tilde{q})^{2}}{36}, \\
a_{3} & =\frac{E_{4}(\tilde{q})\left[77 E_{4}(\tilde{q})^{3}+211 E_{6}(\tilde{q})^{2}\right]}{216}, & b_{3} & =-\frac{E_{6}(\tilde{q})\left[197 E_{4}(\tilde{q})^{3}+91 E_{6}(\tilde{q})^{2}\right]}{216} .
\end{aligned}
$$

The recursion relation for the modular forms $a_{n}$ and $b_{n}$ are

$$
\begin{aligned}
& a_{n+1}=-\left(2 n-\frac{1}{6}\right) E_{6}(\tilde{q}) a_{n}+\frac{1}{6} E_{4}(\tilde{q}) b_{n}+2 E_{4}(\tilde{q})^{\frac{3 n+1}{2}} \partial_{\tilde{t}}\left[\frac{a_{n}}{E_{4}(\tilde{q})^{\frac{3 n-1}{2}}}\right], \\
& b_{n+1}=-\left(2 n+\frac{1}{6}\right) E_{6}(\tilde{q}) b_{n}-\frac{1}{6} E_{4}(\tilde{q})^{2} a_{n}+2 E_{4}(\tilde{q})^{\frac{3 n+2}{2}} \partial_{\tilde{t}}\left[\frac{b_{n}}{E_{4}(\tilde{q})^{\frac{3 n}{2}}}\right] .
\end{aligned}
$$

We then consider the case $c_{0}\left(z_{1}\right)=\tilde{t} E_{4}(\tilde{q})^{\frac{1}{4}}$. From the recursion relation (D.49) we can check inductively that

$$
\prod_{k=0}^{n-1}\left(\theta_{z_{1}}-k\right)\left[\tilde{t} E_{4}(\tilde{q})^{\frac{1}{4}}\right]=\frac{E_{4}(\tilde{q})^{\frac{1}{4}}}{\left(E_{4}(\tilde{q})^{\frac{3}{2}}+E_{6}(\tilde{q})\right)^{n}}\left[\tilde{t}\left(a_{n} E_{2}(\tilde{q})+b_{n}\right)+12 a_{n}\right]
$$


So we find the power series solution and one $\operatorname{logarithmic} \log \left(z_{1}\right)$ solution to the PicardFuchs equations (2.10)

$$
\begin{aligned}
& X_{0}=E_{4}(\tilde{q})^{\frac{1}{4}}\left[1+\sum_{n=1}^{\infty} \frac{a_{3 n} E_{2}(\tilde{q})+b_{3 n}}{2^{3 n}(n !)^{3} E_{4}(\tilde{q})^{\frac{9 n}{2}}} \frac{z_{2}^{n}}{\left(1-432 z_{1}\right)^{3 n}}\right], \\
& X_{1}=E_{4}(\tilde{q})^{\frac{1}{4}}\left[\tilde{t}+\sum_{n=1}^{\infty} \frac{\tilde{t}\left(a_{3 n} E_{2}(\tilde{q})+b_{3 n}\right)+12 a_{3 n}}{2^{3 n}(n !)^{3} E_{4}(\tilde{q})^{\frac{9 n}{2}}} \frac{z_{2}^{n}}{\left(1-432 z_{1}\right)^{3 n}}\right],
\end{aligned}
$$

We compute the flat coordinate of the compact model

$$
\begin{aligned}
t_{1} & =\frac{X_{1}}{X_{0}} \\
& =\tilde{t}+\left[\sum_{n=1}^{\infty} \frac{12 a_{3 n}}{2^{3 n}(n !)^{3} E_{4}(\tilde{q})^{\frac{9 n}{2}}} \frac{z_{2}^{n}}{\left(1-432 z_{1}\right)^{3 n}}\right] /\left[1+\sum_{n=1}^{\infty} \frac{a_{3 n} E_{2}(\tilde{q})+b_{3 n}}{2^{3 n}(n !)^{3} E_{4}(\tilde{q})^{\frac{9 n}{2}}} \frac{z_{2}^{n}}{\left(1-432 z_{1}\right)^{3 n}}\right] .
\end{aligned}
$$

The expression quantifies the deformation of $t_{1}$ away from $\tilde{t}$ as asymptotic expansion in small $z_{2}$, where the coefficients are rational functions of quasi-modular forms of $\tilde{t}$

$$
t_{1}-\tilde{t}=\sum_{n=1}^{\infty} \frac{P_{18 n-2}(\tilde{q})}{E_{4}(\tilde{q})^{\frac{9 n}{2}}} \frac{z_{2}^{n}}{\left(1-432 z_{1}\right)^{3 n}},
$$

where $P_{18 n-2}(\tilde{q})$ are some quasi-modular forms of weight $18 n-2$. We will need the following nice formula

$$
\partial_{E_{2}(\tilde{q})}\left(t_{1}-\tilde{t}\right)^{-1}=\frac{1}{12}
$$

Here we regard $z_{2}$ as free variable which is independent of $\tilde{q}$, so that $\partial_{E_{2}(\tilde{q})} z_{2}=0$.

Before we proceed further, we shall prove a useful general formula on $E_{2}$ derivative. Suppose $P_{k}$ is a rational function of quasi-modular forms, with modular weight $k$, then we have the formula

$$
\partial_{E_{2}} \partial_{t} P_{k}=\partial_{t} \partial_{E_{2}} P_{k}+\frac{k}{12} P_{k}
$$

where we use the notation $t=\log (q)=2 \pi i \tau$. A similar formula was used in [36] to derive of holomorphic anomaly equation in the Nekrasov-Shatashvili limit, and in [37] to studying the relation between holomorphic and modular anomaly equations in the Seiberg-Witten theory. The derivation of (D.55) uses the covariant derivative. It is well known that $E_{2}$ is not a modular form, but with an an-holomorphic shift $\hat{E}_{2}=E_{2}-\frac{6 i}{\pi(\tau-\bar{\tau})}$ it becomes a modular form. To preserve the almost holomorphic structure, we shall use the covariant or Maass derivative

$$
D_{\tau}=\partial_{\tau}+\frac{k}{(\tau-\bar{\tau})}
$$

where $k$ is the modular weight. The derivative with respect to $E_{2}$ can be related to the anti-holomorphic derivative

$$
\bar{\partial}_{\bar{\tau}}=\left(\bar{\partial}_{\bar{\tau}} \hat{E}_{2}\right) \partial_{\hat{E}_{2}}=\frac{6}{\pi i(\tau-\bar{\tau})^{2}} \partial_{\hat{E}_{2}}
$$


We can compute the derivative

$$
\bar{\partial}_{\bar{\tau}} D_{\tau} P_{k}=\left(\partial_{\tau}+\frac{k}{(\tau-\bar{\tau})}\right) \bar{\partial}_{\bar{\tau}} P_{k}+\left(\bar{\partial}_{\bar{\tau}} \frac{k}{(\tau-\bar{\tau})}\right) P_{k}
$$

Taking the holomorphic limit and cancel out the infinitesimal factor $(\tau-\bar{\tau})^{-2}$ we arrive at the formula (D.55).

The above formula (D.55) is valid for any rational function $P_{k}$ of quasi-modular forms, with possible fractional powers, which satisfies the homogeneous relation with modular weight

$$
P_{k}\left(\lambda^{2} E_{2}, \lambda^{4} E_{4}, \lambda^{6} E_{6}\right)=\lambda^{k} P_{k}\left(E_{2}, E_{4}, E_{6}\right) .
$$

However it is a little tricky for the logarithmic function, which does not satisfy the above relation (D.59). Naively the logarithmic function has zero modular weight and the $E_{2}$ derivative should commute with $\tau$ derivative. But this is not the case, as we can easily compute with the formula (D.55),

$$
\partial_{E_{2}} \partial_{t} \log \left(P_{k}\right)=\partial_{t} \partial_{E_{2}} \log \left(P_{k}\right)+\frac{k}{12}
$$

We will not need this logarithmic formula in this subsection, but it will be useful later.

We can take derivative $n$ times on a rational function of quasi-modular forms, and use formula (D.55) to compute

$$
\partial_{E_{2}} \partial_{t}^{n} P_{k}=\partial_{t}^{n} \partial_{E_{2}} P_{k}+\frac{n(k+n-1)}{12} \partial_{t}^{n-1} P_{k}
$$

Suppose $P_{k}\left(q_{1}\right)$ is a rational function with possible fractional powers of quasi-modular forms of $q_{1}$, with modular weight $k$. We can expand it as a power series of $\frac{z_{2}}{(1-432)^{3}}$ with coefficients as rational functions of (quasi)-modular forms of $\tilde{q}$, using the expansion (D.52)

$$
P_{k}\left(q_{1}\right)=\sum_{n=0}^{\infty} \frac{\left(t_{1}-\tilde{t}\right)^{n}}{n !} \partial_{\tilde{t}}^{n} P_{k}(\tilde{q}),
$$

We can compute the derivative of $E_{2}(\tilde{q})$ and use the two formulae (D.54), (D.61)

$$
\begin{aligned}
& \partial_{E_{2}(\tilde{q})} P_{k}\left(q_{1}\right)= \sum_{n=0}^{\infty}\left\{\frac{\left(t_{1}-\tilde{t}\right)^{n-1}}{(n-1) !}\left[\partial_{E_{2}(\tilde{q})}\left(t_{1}-\tilde{t}\right)\right] \partial_{\tilde{t}}^{n} P_{k}(\tilde{q})+\frac{\left(t_{1}-\tilde{t}\right)^{n}}{n !} \partial_{E_{2}(\tilde{q})} \partial_{\tilde{t}}^{n} P_{k}(\tilde{q})\right\} \\
&= \sum_{n=0}^{\infty}\left\{-\frac{1}{12} \frac{\left(t_{1}-\tilde{t}\right)^{n+1}}{(n-1) !} \partial_{\tilde{t}}^{n} P_{k}(\tilde{q})\right. \\
&\left.\quad+\frac{\left(t_{1}-\tilde{t}\right)^{n}}{n !}\left[\partial_{\tilde{t}}^{n} \partial_{E_{2}(\tilde{q})} P_{k}(\tilde{q})+\frac{n(k+n-1)}{12} \partial_{\tilde{t}}^{n-1} P_{k}(\tilde{q})\right]\right\}, \\
&=\partial_{E_{2}\left(q_{1}\right)} P_{k}\left(q_{1}\right)+\frac{k}{12}\left(t_{1}-\tilde{t}\right) P_{k}\left(q_{1}\right) .
\end{aligned}
$$

We see the derivative vanishes when $P_{k}\left(q_{1}\right)$ has zero modular weight $k=0$ and is modular, i.e. no $E_{2}$ dependence. 
Now we are have the necessary ingredients for proving the conjecture (2). First we consider the case of $1-864 z_{1}=\frac{E_{6}(\tilde{q})}{E_{4}(\tilde{q})^{\frac{3}{2}}}$. We shall expand in terms of the modular parameter $q_{1}$ of the compact model

$$
\frac{E_{6}(\tilde{q})}{E_{4}(\tilde{q})^{\frac{3}{2}}}=\frac{1}{E_{4}\left(q_{1}\right)^{\frac{3}{2}}}\left[E_{6}\left(q_{1}\right)+\sum_{n=1}^{\infty} \frac{f_{n}\left(q_{1}\right)}{E_{4}\left(q_{1}\right)^{\frac{9 n}{2}}} \frac{z_{2}^{n}}{\left(1-432 z_{1}\right)^{3 n}}\right],
$$

We can determine the coefficients $f_{n}$ recursively by expanding the modular forms of $q_{1}$ in terms of $\tilde{q}$ and compare with the left hand side. It is clear that $f_{n}\left(q_{1}\right)$ are rational functions of quasi-modular forms of $q_{1}$ of modular weight $18 n+6$, with possible negative powers of $E_{4}$, but no fractional powers. To show it has no $E_{2}$ dependence, we take derivative of $E_{2}(\tilde{q})$ on both sides and use the formula (D.63)

$$
0=\sum_{n=1}^{\infty} \frac{\partial_{E_{2}\left(q_{1}\right)} f_{n}\left(q_{1}\right)}{E_{4}\left(q_{1}\right)^{\frac{9 n+3}{2}}} \frac{z_{2}^{n}}{\left(1-432 z_{1}\right)^{3 n}}
$$

where we have use the fact that the coefficients have zero modular weight. Since $z_{2}$ is a free variable, we find $\partial_{E_{2}\left(q_{1}\right)} f_{n}\left(q_{1}\right)=0$, i.e. it is modular.

We also need to check that there is no negative powers of $E_{4}\left(q_{1}\right)$ in $f_{n}\left(q_{1}\right)$. From the expansion (D.53) we see that the coefficients of $\frac{z_{2}^{n}}{\left(1-432 z_{1}\right)^{3 n}}$ always have the factor of $E_{4}\left(q_{1}\right)^{-\frac{9 n}{2}}$. There could be also negative powers of $E_{4}$ from the derivative $\partial_{\tilde{t}}$ in (D.62) since each derivative increases the negative power of $E_{4}$ by one. However, we note that since $a_{n}$ has modular weight is $6 n-2$, it can not be purely a power of $E_{6}(\tilde{q})$ and always has a factor $E_{4}(\tilde{q})$. So the $\left(t_{1}-\tilde{t}\right)^{n}$ factor in $(\mathrm{D} .62)$ contributes a factor $E_{4}(\tilde{q})^{n}$ which cancel the one from taking derivative. Therefore $f_{n}\left(q_{1}\right)$ is a modular form.

Furthermore, since the derivative $\partial_{\tilde{t}}^{n}\left[\frac{E_{6}(\tilde{q})}{E_{4}(\tilde{q})^{\frac{3}{2}}}\right]$ always contains a factor of $E_{4}(\tilde{q})^{3}-E_{6}(\tilde{q})^{2}$, we see that the the coefficient $f_{n}\left(q_{1}\right)$ always have a factor of $E_{4}\left(q_{1}\right)^{3}-E_{6}\left(q_{1}\right)^{2}$, as confirmed by the explicit expansion in (D.26). According to the discussion in equations (D.29), (D.30), we immediately infer the modularity of the expansion coefficients of $z_{1}\left(1-432 z_{1}\right)$.

Alternatively, we can also expand $z_{1}\left(1-432 z_{1}\right)=\frac{E_{4}(\tilde{q})^{3}-E_{6}(\tilde{q})^{2}}{1728 E_{4}(\tilde{q})^{3}}$ in the same way as $1-864 z_{1}$ in (D.64). From the same reasoning as above we can also see the modularity of the expansion (D.24). We note that the derivative $\partial_{\tilde{t}}^{n}\left[E_{4}(\tilde{q})^{3}-E_{6}(\tilde{q})^{2}\right]$ also always contains a factor of $E_{4}(\tilde{q})^{3}-E_{6}(\tilde{q})^{2}$, and it is factored out in this case in the expansion (D.24), so that the higher order coefficients do not necessarily contain the factor.

Finally we consider the case of $X_{0}$, which is a little trickier than the previous two cases since the modular weight is not zero. Again we make the ansatz in terms of the modular parameter $q_{1}$ of the compact model

$$
X_{0}=E_{4}\left(q_{1}\right)^{\frac{1}{4}}\left[1+\sum_{n=1}^{\infty} \frac{f_{n}\left(q_{1}\right)}{E_{4}\left(q_{1}\right)^{\frac{9 n}{2}}} \frac{z_{2}^{n}}{\left(1-432 z_{1}\right)^{3 n}}\right],
$$

and determine $f_{n}\left(q_{1}\right)$ recursively comparing with the solution (D.51) and using the deformation (D.53). From the same reasonings above we see $f_{n}\left(q_{1}\right)$ contains no negative power 
of $E_{4}\left(q_{1}\right)$ and no fractional powers, so it is a quasi-modular form. We only need to show it is $E_{2}$ independent. We compute the $E_{2}(\tilde{q})$ derivative in the $X_{0}$ solution in (D.51)

$$
\begin{aligned}
\partial_{E_{2}(\tilde{q})} X_{0} & =E_{4}(\tilde{q})^{\frac{1}{4}}\left[\sum_{n=1}^{\infty} \frac{a_{3 n}}{2^{3 n}(n !)^{3} E_{4}(\tilde{q})^{\frac{9 n}{2}}} \frac{z_{2}^{n}}{\left(1-432 z_{1}\right)^{3 n}}\right] \\
& =\frac{t_{1}-\tilde{t}}{12} X_{0},
\end{aligned}
$$

where in the last step we use (D.52). On the other hand, we can also compute the $E_{2}(\tilde{q})$ derivative using the ansatz (D.66) and the formula (D.63) with the modular weight $k=1$, and we get

$$
\partial_{E_{2}(\tilde{q})} X_{0}=\frac{t_{1}-\tilde{t}}{12} X_{0}+\sum_{n=1}^{\infty} \frac{\partial_{E_{2}\left(q_{1}\right)} f_{n}\left(q_{1}\right)}{E_{4}\left(q_{1}\right)^{\frac{18 n-1}{4}}} \frac{z_{2}^{n}}{\left(1-432 z_{1}\right)^{3 n}} .
$$

Comparing the two results (D.67), (D.68) we find that $f_{n}\left(q_{1}\right)$ has no $E_{2}$ dependence. Thus we have proven Conjecture 2. We see the case of $X_{0}$ is quite interesting. It is quasimodular when we use the $\tilde{q}$ parameter, but the $E_{2}$ dependence vanishes and it becomes purely modular when we use the physical Kähler parameter $q_{1}$ of the compact model.

We can also show that the modular forms $f_{n}\left(q_{1}\right)$ in (D.66) contain a factor of $\eta^{24} \sim$ $E_{4}^{3}-E_{6}^{2}$, as can be seen from the explicit formulae in the expansion (D.27). Since $f_{n}\left(q_{1}\right)$ is a modular form, it is sufficient to show that $f_{n}\left(q_{1}\right)=0$ at $q_{1}=0$ in order to be divisible by $\eta^{24}\left(q_{1}\right)$. We note that the derivative of any rational function of modular forms and their fractional powers vanish at $q_{1}$, as long as it is not singular at $q_{1}=0$, due to its $q$-series expansion. So the contributions to $f_{n}(0)$ from lower order terms in (D.66) vanish. We also need to evaluate the solution (D.51) for $X_{0}$ at $\tilde{q}=0$. We denote the value of $a_{n}(\tilde{q}), b_{n}(\tilde{q})$ in the solution at $\tilde{q}=0$ simply as $a_{n}(0), b_{n}(0)$. The recursion relation (D.49) at $\tilde{q}=0$ is

$$
\begin{aligned}
& a_{n+1}(0)=-\left(2 n-\frac{1}{6}\right) a_{n}(0)+\frac{1}{6} b_{n}(0), \\
& b_{n+1}(0)=-\left(2 n+\frac{1}{6}\right) b_{n}(0)-\frac{1}{6} a_{n}(0) .
\end{aligned}
$$

Adding together we find

$$
a_{n+1}(0)+b_{n+1}(0)=-2 n\left[a_{n}(0)+b_{n}(0)\right],
$$

so the sum $a_{n}(0)+b_{n}(0)$ vanishes for $n \geq 1$. The solution is simply $X_{0}=1$ for $\tilde{q}=0$, and does not contribute to higher order terms either. Thus $f_{n}\left(q_{1}\right)$ vanish at $q_{1}=0$, i.e. is divisible by $\eta^{24}\left(q_{1}\right)$.

Next we would like to find the solution to Picard-Fuchs equation with leading $\log \left(z_{2}\right)$ behavior. We make the ansatz

$$
X_{2}=X_{0}\left[\log \left(z_{2}\right)-\frac{3}{2} t_{1}-\frac{3}{2} \log \left(\frac{1-432 z_{1}}{z_{1}}\right)\right]+\sum_{n=0}^{\infty} \alpha_{n}\left(z_{1}\right) z_{2}^{n},
$$

where $X_{0}$ and $X_{0} t_{1}=X_{1}$ are the two solutions found previously in (D.51). The terms $\frac{3}{2} t_{1}+\frac{3}{2} \log \left(\frac{1-432 z_{1}}{z_{1}}\right)$ comes from (D.11) which is the solution to the $z_{2}$ zero order equation 
with the correct leading asymptotic behavior, so that we can choose the initial function $\alpha_{0}\left(z_{1}\right)=0$. For convenience, we denote

$$
d_{n}=\frac{E_{4}(\tilde{q})^{\frac{1}{4}}}{\left(E_{4}(\tilde{q})^{\frac{3}{2}}+E_{6}(\tilde{q})\right)^{n}}\left(a_{n} E_{2}(\tilde{q})+b_{n}\right),
$$

where $a_{n}, b_{n}$ are modular forms defined in (D.48), and satisfy the recursion relation (D.49), so that the power series solution

$$
X_{0}=\sum_{n=0}^{\infty} \frac{d_{3 n}}{(n !)^{3}} z_{2}^{n}
$$

Again after some calculations, we find recursion relation for $\alpha_{n}\left(z_{1}\right)$ in (D.71) from the second Picard-Fuchs equation

$$
\begin{aligned}
\alpha_{n+1}\left(z_{1}\right)= & \frac{1}{(n+1)^{3}} \prod_{i=0}^{2}\left(\theta_{1}-3 n-i\right) \alpha_{n}\left(z_{1}\right)-\frac{3 d_{3 n+3}}{(n+1)(n+1) !^{3}} \\
& -\frac{9 E_{6}(\tilde{q}) d_{3 n+2}}{(n+1) !^{3}\left(E_{4}(\tilde{q})^{\frac{3}{2}}+E_{6}(\tilde{q})\right)}+\frac{9\left(E_{4}(\tilde{q})^{3}+E_{6}(\tilde{q})^{2}\right) d_{3 n+1}}{(n+1) !^{3}\left(E_{4}(\tilde{q})^{\frac{3}{2}}+E_{6}(\tilde{q})\right)^{2}} \\
& -\frac{6 E_{6}(\tilde{q})\left(3 E_{4}(\tilde{q})^{3}+E_{6}(\tilde{q})^{2}\right) d_{3 n}}{(n+1) !^{3}\left(E_{4}(\tilde{q})^{\frac{3}{2}}+E_{6}(\tilde{q})\right)^{3}}
\end{aligned}
$$

From the recursion relation and initial condition $\alpha_{0}=0$, it is clear that the coefficients $\alpha_{n}\left(z_{1}\right)$ have the following structure

$$
\alpha_{n}=\frac{E_{4}(\tilde{q})^{\frac{1}{4}}}{\left(E_{4}(\tilde{q})^{\frac{3}{2}}+E_{6}(\tilde{q})\right)^{3 n}}\left(A_{n}(\tilde{q}) E_{2}(\tilde{q})+B_{n}(\tilde{q})\right),
$$

where $A_{n}(\tilde{q}), B_{n}(\tilde{q})$ are modular forms of $\tilde{q}$ of weight $18 n-2,18 n$. Some low order formulae are

$$
\begin{aligned}
& A_{1}=\frac{E_{4}\left(31 E_{4}^{3}+113 E_{6}^{2}\right)}{72}, \quad B_{1}=-\frac{E_{6}\left(1297 E_{4}^{3}+575 E_{6}^{2}\right)}{72}, \\
& A_{2}=-\frac{E_{4} E_{6}}{6912}\left(233753 E_{4}^{6}+812750 E_{4}^{3} E_{6}^{2}+252953 E_{6}^{4}\right), \\
& B_{2}=\frac{1}{82944}\left(3717955 E_{4}^{9}+59441331 E_{4}^{6} E_{6}^{2}+66006225 E_{4}^{3} E_{6}^{4}+5867321 E_{6}^{6}\right) .
\end{aligned}
$$

The second flat coordinate is then

$$
t_{2}=\frac{X_{2}}{X_{0}}=\log \left(z_{2}\right)-\frac{3}{2} t_{1}-\frac{3}{2} \log \left(\frac{1-432 z_{1}}{z_{1}}\right)+\frac{1}{X_{0}} \sum_{n=1}^{\infty} \alpha_{n}\left(z_{1}\right) z_{2}^{n},
$$

where $\alpha_{n}$ are expressed in (D.75) in terms of the modular forms $A_{n}$ and $B_{n}$. It is clear we can write a series expansion

$$
\log (Q)=\log (Z)+\sum_{n=1}^{\infty} f_{n}\left(q_{1}\right) Z^{n}
$$


where $Q=\frac{\exp \left(t_{2}+\frac{3 t_{2}}{2}\right)}{\eta\left(q_{1}\right)^{36}}, Z=\frac{z_{2}}{\left(1-432 z_{1}\right)^{3} E_{4}\left(q_{1}\right)^{\frac{9}{2}}}$ are the variables defined in (D.32), (D.23). Using the relation between $t_{1}$ and $\tilde{t}$ as in equation (D.53) we see that $f_{n}\left(q_{1}\right)$ here must be quasi-modular forms of weight $18 n$. Because the coefficients of $z_{2}^{n}$ have zero modular weight, the derivative of $E_{2}(\tilde{q})$ and $E_{2}\left(q_{1}\right)$ are the the same for $\log (Q)$ according to the formula (D.63). In order to prove conjecture (3), we should confirm the equation (D.40). We compute the $E_{2}$ derivative

$$
\begin{aligned}
\partial_{E_{2}\left(q_{1}\right)} \log (Q) & =\partial_{E_{2}(\tilde{q})} \log (Q) \\
& =-\frac{t_{1}-\tilde{t}}{12 X_{0}} \sum_{n=1}^{\infty} \alpha_{n}\left(z_{1}\right) z_{2}^{n}+\frac{1}{X_{0}} \sum_{n=1}^{\infty} \frac{E_{4}(\tilde{q})^{\frac{1}{4}} A_{n}(\tilde{q})}{\left(E_{4}(\tilde{q})^{\frac{3}{2}}+E_{6}(\tilde{q})\right)^{3 n}} z_{2}^{n},
\end{aligned}
$$

where we have used (D.67). We shall prove the above equation is equal to the $\frac{1}{12} \partial_{t_{2}} \mathcal{F}_{\text {inst }}^{(0)}\left(t_{1}, t_{2}\right)$, where $\mathcal{F}_{\text {inst }}^{(0)}$ is the instanton part of the the prepotential.

The classical contribution in the prepotential for our model is

$$
\mathcal{F}_{\text {classical }}^{(0)}=\frac{3 t_{1}^{3}}{2}+\frac{3}{2} t_{1}^{2} t_{2}+\frac{1}{2} t_{1} t_{2}^{2}
$$

The prepotential is determined by the fact that $X_{0} \partial_{t_{i}} \mathcal{F}^{(0)}$ are the double logarithmic solutions of the Picard-Fuchs (PF) equations (2.10). In order to confirm the equation (D.40), we shall show the following is a solution to the PF equations

$$
\begin{aligned}
w_{3} & =X_{0}\left(\frac{3}{2} t_{1}^{2}+t_{1} t_{2}\right)-\left(t_{1}-\tilde{t}\right) \sum_{n=1}^{\infty} \alpha_{n}\left(z_{1}\right) z_{2}^{n}+12 \sum_{n=1}^{\infty} \frac{E_{4}(\tilde{q})^{\frac{1}{4}} A_{n}(\tilde{q})}{\left(E_{4}(\tilde{q})^{\frac{3}{2}}+E_{6}(\tilde{q})\right)^{3 n}} z_{2}^{n} \quad \text { (D.81) } \\
& =X_{1}\left[\log \left(z_{2}\right)-\frac{3}{2} \log \left(\frac{1-432 z_{1}}{z_{1}}\right)\right]+\tilde{t} \sum_{n=1}^{\infty} \alpha_{n}\left(z_{1}\right) z_{2}^{n}+12 \sum_{n=1}^{\infty} \frac{E_{4}(\tilde{q})^{\frac{1}{4}} A_{n}(\tilde{q})}{\left(E_{4}(\tilde{q})^{\frac{3}{2}}+E_{6}(\tilde{q})\right)^{3 n}} z_{2}^{n},
\end{aligned}
$$

where we have use the solution for $X_{2}$ in (D.71).

After some tedious calculations, we check that (D.81) is indeed a solution. We can provide some details in the following. At zero order $z_{2}$, we need to check the equation from the first PF operator

$$
\left[\theta_{1}^{2}-12 z_{1}\left(6 \theta_{1}+1\right)\left(6 \theta_{1}+5\right)\right]\left[-\frac{3}{2} \tilde{t} E_{4}(\tilde{q})^{\frac{1}{4}} \log \left(\frac{1-432 z_{1}}{z_{1}}\right)\right]=3 \theta_{1}\left[\tilde{t} E_{4}(\tilde{q})^{\frac{1}{4}}\right] .
$$

Assuming the two Picard-Fuchs equations are consistent, we only need to further check the second $\mathrm{PF}$ equation which provide recursion relation for the coefficients of $z_{2}^{n}$. We denote the coefficient of $z_{2}^{n}$ in the solution (D.81) as

$$
\beta_{n}=\tilde{t} \alpha_{n}+\frac{12 E_{4}(\tilde{q})^{\frac{1}{4}} A_{n}(\tilde{q})}{\left(E_{4}(\tilde{q})^{\frac{3}{2}}+E_{6}(\tilde{q})\right)^{3 n}} .
$$

We see the similar structure for the ansatz of $X_{2}$ in (D.71). From the same calculations we derive the recursion relation for $\beta_{n}\left(z_{1}\right)$ from the second Picard-Fuchs equation, similar 
to (D.74),

$$
\begin{aligned}
\beta_{n+1}\left(z_{1}\right)= & \frac{1}{(n+1)^{3}} \prod_{i=0}^{2}\left(\theta_{1}-3 n-i\right) \beta_{n}\left(z_{1}\right)-\frac{3 e_{3 n+3}}{(n+1)(n+1) !^{3}} \\
& -\frac{9 E_{6}(\tilde{q}) e_{3 n+2}}{(n+1) !^{3}\left(E_{4}(\tilde{q})^{\frac{3}{2}}+E_{6}(\tilde{q})\right)}+\frac{9\left(E_{4}(\tilde{q})^{3}+E_{6}(\tilde{q})^{2}\right) e_{3 n+1}}{(n+1) !^{3}\left(E_{4}(\tilde{q})^{\frac{3}{2}}+E_{6}(\tilde{q})\right)^{2}} \\
& -\frac{6 E_{6}(\tilde{q})\left(3 E_{4}(\tilde{q})^{3}+E_{6}(\tilde{q})^{2}\right) e_{3 n}}{(n+1) !^{3}\left(E_{4}(\tilde{q})^{\frac{3}{2}}+E_{6}(\tilde{q})\right)^{3}}
\end{aligned}
$$

where we denote

$$
e_{n}=\frac{E_{4}(\tilde{q})^{\frac{1}{4}}}{\left(E_{4}(\tilde{q})^{\frac{3}{2}}+E_{6}(\tilde{q})\right)^{n}}\left[\tilde{t}\left(a_{n} E_{2}(\tilde{q})+b_{n}\right)+12 a_{n}\right],
$$

so that the solution $X_{1}=\sum_{n=0}^{\infty} \frac{e_{3 n}}{n !^{3}} z_{2}^{n}$. We further denote

$$
\frac{E_{4}(\tilde{q})^{\frac{1}{4}}\left[A_{n}^{(k)} E_{2}(\tilde{q})+B_{n}^{(k)}\right]}{\left(E_{4}(\tilde{q})^{\frac{3}{2}}+E_{6}(\tilde{q})\right)^{3 n+k+1}}=\prod_{i=0}^{k}\left(\theta_{1}-3 n-i\right) \alpha_{n},
$$

where $A_{n}^{(k)}$ and $B_{n}^{(k)}$ are modular forms of $\tilde{q}$ of weight $6(3 n+k+1)-2$ and $6(3 n+k+1)$. Then similar to the case of solution $X_{1}$, one can show inductively

$$
\frac{E_{4}(\tilde{q})^{\frac{1}{4}}\left[\tilde{t}\left(A_{n}^{(k)} E_{2}(\tilde{q})+B_{n}^{(k)}\right)+12 A_{n}^{(k)}\right]}{\left(E_{4}(\tilde{q})^{\frac{3}{2}}+E_{6}(\tilde{q})\right)^{3 n+k+1}}=\prod_{i=0}^{k}\left(\theta_{1}-3 n-i\right)\left(\tilde{t} \alpha_{n}+\frac{12 E_{4}(\tilde{q})^{\frac{1}{4}} A_{n}(\tilde{q})}{\left(E_{4}(\tilde{q})^{\frac{3}{2}}+E_{6}(\tilde{q})\right)^{3 n}}\right) .
$$

¿From the recursion relation for $\alpha_{n}$ in (D.74), we can write the recursion separately for $A_{n}$ and $B_{n}$ as

$$
\begin{aligned}
A_{n+1}= & \frac{A_{n}^{(2)}}{(n+1)^{3}}-\frac{3 a_{3 n+3}}{(n+1)(n+1) !^{3}}-\frac{9 E_{6}(\tilde{q}) a_{3 n+2}}{(n+1) !^{3}}+\frac{9\left(E_{4}(\tilde{q})^{3}+E_{6}(\tilde{q})^{2}\right) a_{3 n+1}}{(n+1) !^{3}} \\
& -\frac{6 E_{6}(\tilde{q})\left(3 E_{4}(\tilde{q})^{3}+E_{6}(\tilde{q})^{2}\right) a_{3 n}}{(n+1) !^{3}}, \\
B_{n+1}= & \frac{B_{n}^{(2)}}{(n+1)^{3}}-\frac{3 b_{3 n+3}}{(n+1)(n+1) !^{3}}-\frac{9 E_{6}(\tilde{q}) b_{3 n+2}}{(n+1) !^{3}}+\frac{9\left(E_{4}(\tilde{q})^{3}+E_{6}(\tilde{q})^{2}\right) b_{3 n+1}}{(n+1) !^{3}} \\
& -\frac{6 E_{6}(\tilde{q})\left(3 E_{4}(\tilde{q})^{3}+E_{6}(\tilde{q})^{2}\right) b_{3 n}}{(n+1) !^{3}} .
\end{aligned}
$$

Now it is straightforward to check that $\beta_{n}$ in (D.83) satisfy the recursion relation (D.84). This completes the proof of Conjecture 3 .

\section{E Expansion around a point on the conifold divisor}

We now study the expansion of the higher genus topological string amplitudes around a point on the conifold divisor in terms of the local flat coordinates, and use the gap condition to fix the holomorphic ambiguity. Since the involution symmetry exchanges the 
two conifold divisors, it is sufficient to consider the simpler one $\Delta_{2}=1+27 z_{2}$. The simplest point to consider is the intersection point of the divisor $z_{1}=0$ and $\Delta_{2}=1+27 z_{2}$. This is a normal intersection, and we can choose the local coordinates as

$$
\left(z_{c 1}, z_{c 2}\right)=\left(z_{1}, z_{2}+\frac{1}{27}\right)
$$

We solve the PF equations (2.10) perturbatively around this point. There should be 6 linearly independent solutions. We only need 3 of them with at most one logarithmic leading term in order to compute the flat coordinates. The three solutions are two power series solutions $X_{0}, X_{1}$ and one logarithmic solution $X_{2}$ as the following

$$
\begin{aligned}
X_{0}= & {\left[1+60 z_{c 1}+13860 z_{c 1}^{2}+\frac{9529520 z_{c 1}^{3}}{3}+148728580 z_{c 1}^{4}-569333004240 z_{c 1}^{5}\right.} \\
& \left.-\frac{5035405372197200 z_{c 1}^{6}}{9}+\mathcal{O}\left(z_{c 1}^{7}\right)\right]+\left[24504480 z_{c 1}^{3}+32125373280 z_{c 1}^{4}\right. \\
& \left.+27949074753600 z_{c 1}^{5}+19093358660376000 z_{c 1}^{6}+\mathcal{O}\left(z_{c 1}^{7}\right)\right] z_{c 2} \\
& +\left[15162373053828000 z_{c 1}^{6}+\mathcal{O}\left(z_{c 1}^{7}\right)\right] z_{c 2}^{2}+\mathcal{O}\left(z_{c 2}^{3}\right) \\
X_{1}= & z_{c 2}+\left(\frac{33}{2}+270 z_{c 1}\right) z_{c 2}^{2}+\left(327+7020 z_{c 1}+374220 z_{c 1}^{2}\right) z_{c 2}^{3}+\mathcal{O}\left(z_{c 2}^{4}\right) \\
X_{2}= & X_{0} \log \left(z_{c 1}\right)+\left[372 z_{c 1}+98442 z_{c 1}^{2}+\frac{224976472 z_{c 1}^{3}}{9}+\frac{7098994823 z_{c 1}^{4}}{3}+\mathcal{O}\left(z_{c 1}^{5}\right)\right] \\
& +\left[-540 z_{c 1}-124740 z_{c 1}^{2}+132742656 z_{c 1}^{3}+220660710516 z_{c 1}^{4}+\mathcal{O}\left(z_{c 1}^{5}\right)\right] z_{c 2} \\
& +\left[27-4860 z_{c 1}-1683990 z_{c 1}^{2}-606485880 z_{c 1}^{3}-307198881990 z_{c 1}^{4}+\mathcal{O}\left(z_{c 1}^{5}\right)\right] z_{c 2}^{2} \\
& +\mathcal{O}\left(z_{c 2}^{3}\right)
\end{aligned}
$$

The local flat coordinates are computed as the ratio of the solutions $t_{c 1}=\frac{X_{1}}{X_{0}}$ and $t_{c 2}=\frac{X_{2}}{X_{0}}$. For the power series solution, the flat coordinate goes like $t_{c 1} \sim z_{c 2}=z_{2}+\frac{1}{27}$, which is the expected asymptotic behavior of near a conifold point. For the logarithmic solution, the flat coordinate goes like $t_{c 2} \sim \log \left(z_{c 1}\right)$, which is similar to the behavior near large volume point, and we should use the exponential of the flat coordinate as the expansion parameter. So we define the expansion parameters in terms of flat coordinates

$$
t_{c}:=t_{c 1}=\frac{X_{1}}{X_{0}}, \quad q_{c}:=\exp \left(t_{c 2}\right)
$$

We shall compute the asymptotic expansion of the propagators $S^{i j}, S^{i}, S$, where we have written the propagators in $z_{i}$ coordinate and for simplicity we denote $S^{i j}=S^{z_{i} z_{j}}$ and $S^{i}=S^{z_{i}}$. Here the propagators should simply transform as tensors. In the case of involution symmetry, there is a minus sign and shifts for $S^{i}$ and $S$ because the involution map the point to a different branch of moduli space. But this should not happen locally. The three point function also transforms as tensor without minus sign. The equations (B.2) 
are coordinate invariant if we take the holomorphic ambiguities $h_{i}^{j k}, h_{i}^{j}, h_{i}, h_{i j}$ to transform as tensors, while the Christoffel symbol $\Gamma_{i j}^{k}$ and the holomorphic ambiguity $s_{i j}^{k}$ transforms with a shift according to the well known transformation rule of Christoffel symbol as in (B.4).

We can compute the asymptotic expansion of the Christoffel symbol $\Gamma_{z_{c i} z_{c j}}^{z_{c k}}$ in the local coordinates (E.1) by the well known formula from special geometry $\Gamma_{z_{c i} z_{c j}}^{z_{c k}}=\frac{\partial z_{c k}}{\partial t_{c l}} \frac{\partial^{2} t_{c l}}{\partial z_{c i} \partial z_{c j}}$ in the holomorphic limit, and compute the propagators $S^{z_{c i} z_{c j}}, S^{z_{c i}}, S$ according to (B.4). Here the transformation between the coordinates $z_{c i}$ and $z_{i}$ are particularly simple because the Jacobian transformation matrix $\frac{\partial z_{c i}}{\partial z_{j}}$ is an identity matrix. So the propagators $S^{i j}, S^{i}, S$ and holomorphic ambiguities $h_{i}^{j k}, h_{i}^{j}, h_{i}, h_{i j}, s_{i j}^{k}$ are actually invariant under the coordinate transformation (E.1).

We find that after including the well known scaling factor $X_{0}^{2 g-2}$, the asymptotic expansion of the higher genus topological string amplitudes with the correct holomorphic ambiguity satisfy the gap condition near the point $\left(z_{1}, z_{2}\right)=\left(0,-\frac{1}{27}\right)$. Specifically, we find

$$
X_{0}^{2 g-2} \mathcal{F}^{(g)}=\frac{B_{2 g}}{4 g(g-1) 3^{5(g-1)}} \frac{1}{t_{c}^{2(g-1)}}+\sum_{n=0}^{\infty} f_{n}\left(q_{c}\right) t_{c}^{n}
$$

where $f_{n}\left(q_{c}\right)$ are some power series of $q_{c}$.

Now we can study how the gap condition (E.6) fixes holomorphic ambiguity at higher genus. First as in previous situations, suppose we have found a particular holomorphic ambiguity at genus $g$ such that the gap condition (E.6) is satisfied. Then we may add an additional piece of rational function of $z_{1}, z_{2}$ to $\mathcal{F}^{(g)}$ such that the gap condition (E.6) is not affected. Since near the point $\left(z_{1}, z_{2}\right)=\left(0,-\frac{1}{27}\right)$, we have the asymptotic behavior $X_{0} \sim 1$ and $t_{c} \sim z_{c 2} \sim \Delta_{2}$, this additional rational function should have no pole at the conifold divisor $\Delta_{2}$ in order to be regular at $t_{c} \sim 0$. Together with the involution symmetry as defined by the spaces in (C.2), we consider the following linear space of holomorphic ambiguity

$$
X^{(g, m)}:=\left\{f \mid f \in \sum_{n=0}^{\infty} \oplus V_{2}^{(g, m, n)} \text { and has no pole at } \Delta_{2}\right\} .
$$

In particular, in the case of $m=\left[\frac{19}{3}(g-1)\right]$, according to the proposition (3), the dimension of $X^{\left(g,\left[\frac{19}{3}(g-1)\right]\right)}$ is exactly the remaining number of unknown constants in the genus $g$ holomorphic ambiguity after we impose both the fiber modularity and the gap conditions.

Clearly if $f \in X^{(g, m)}$, then its involution transformation $\tilde{f}=(-1)^{g-1} f \in X^{(g, m)}$ as well. Since the involution transformation symmetry exchanges the two conifold divisors, we infer that $f$ must have no pole at $\Delta_{1}$ either, so it is actually a polynomial of $z_{1}, z_{2}$. Canceling the pole $\Delta_{1}^{2 g-2}$ reduces the degree of $z_{1}$ in the numerator by $6(g-1)$. So the space can be determined as

$$
X^{(g, m)}= \begin{cases}\sum_{n=0}^{\infty} \oplus\left(V_{-}^{(1, m-6(g-1), 2 n)} \oplus V_{+}^{(1, m-6(g-1), 2 n+1)}\right), & \text { if } g \text { is even } \\ \sum_{n=0}^{\infty} \oplus\left(V_{+}^{(1, m-6(g-1), 2 n)} \oplus V_{-}^{(1, m-6(g-1), 2 n+1)}\right), & \text { if } g \text { is odd }\end{cases}
$$


It is now easy to compute the dimension of $X^{(g, m)}$ with the formulae (C.13). We can consider in particular the case of $m=\left[\frac{19}{3}(g-1)\right]$, and find the number of remaining unknown constants at low genus $\operatorname{Dim}\left(X^{\left(g,\left[\frac{19}{3}(g-1)\right]\right)}\right)=0,1,1,1,1,2,1,2,3,2,3$ for $g=2,3, \cdots, 12$ respectively.

We can also estimate the asymptotic behavior of $\operatorname{Dim}\left(X^{\left(g,\left[\frac{19}{3}(g-1)\right]\right)}\right)$ at large genus. Ignoring sub-leading contributions, we find

$$
\operatorname{Dim}\left(X^{\left(g,\left[\frac{19}{3}(g-1)\right]\right)}\right)=\sum_{n=0}^{\frac{g-1}{9}}\left(\frac{g-1}{3}-3 n\right) \sim \frac{g^{2}}{54} .
$$

Since the elements of the space $X^{(g, m)}$ are regular at both conifold divisors $\Delta_{1}, \Delta_{2}$, they would not affect the gap conditions at any other points on the conifold divisors. For example, studying the expansion of topological string amplitudes around the intersection of $\Delta_{1}$ and $\Delta_{2}$ would not give more boundary conditions to fix the holomorphic ambiguity. It is thus sufficient to consider the gap condition around only one point $\left(z_{1}, z_{2}\right)=\left(0,-\frac{1}{27}\right)$ here.

At low genus we can fix the remaining holomorphic ambiguity (E.8) by the boundary conditions at large volume point $\left(z_{1}, z_{2}\right)=(0,0)$, which include the known higher genus constant map contributions and some vanishing property of GV invariants. For example, the GV invariants vanish $n_{d_{E}, d_{B}}^{g}=0$ for base degree $d_{B}=0$ and $g \geq 2$ [3]. We see that only the holomorphic ambiguities with zero $z_{2}$ degree in the space (E.8) contribute to the zero base degree GV invariants. So this property alone can eliminate the subspace $V_{ \pm}^{\left(1,\left[\frac{g-1}{3}\right], 0\right)}$ in the remaining holomorphic ambiguities, which are now actually completely fixed up to genus $g \leq 9$.

We consider also the local limit. The topological string amplitudes on local $\mathbb{P}^{2}$ model which has only the base class are solvable to arbitrary high genus. So we known the zero fiber degree GV invariants $n_{d_{E}, d_{B}}^{g}$ for $d_{E}=0$ from local model calculations, which may fix some more holomorphic ambiguity for the compact model. However, at a closer look we find that the local limit actually does not help. It is easy to check that except one element which is the constant in $V_{+}^{(1, m-6(g-1), 0)}$ or $1-864 z_{1}$ in $V_{-}^{(1, m-6(g-1), 0)}$, all other elements in $X^{(g, m)}$ are polynomially divisible by $z_{1}$, so they do not affect the zero fiber degree GV invariants. So the local limit only fix the constant or $1-864 z_{1}$ in $X^{(g, m)}$, which is already fixed by the known constant map contribution in Gromov-Witten theory.

We can discuss a little more detail on fixing the holomorphic ambiguity with GV invariants. From the definition of the spaces $V_{ \pm}$in (C.10), (C.11), we find that the elements in $V_{ \pm}^{(1, m-6(g-1), n)}$ are polynomially divisible by $z_{1}^{3 n}$, so they only affect the GV invariants $n_{d_{E}, d_{B}}^{g}$ with fiber degree $d_{E} \geq 3 n$. So in order to fix the elements in $V_{ \pm}^{(1, m-6(g-1), n)}$ we need to use the GV invariants $n_{d_{E}, d_{B}}^{g}$ with fiber degree $d_{E} \geq 3 n$ and $d_{B} \geq n$.

These B-model considerations are combined with the use of weak Jacobi forms to fix the topological string amplitudes. Suppose we have the exact formulas up to a base degree $d_{B}$ which are valid for all genus and fiber class, then in the following we shall show that we have sufficient boundary conditions to fix the B-model formula up to any genus no bigger 
than $9\left(d_{B}+1\right)$. To see this, we further define a vector space

$$
Y^{\left(g, d_{B}\right)}=\sum_{n=d_{B}+1}^{\infty} \oplus V_{(-1)^{g+n+1}}^{\left(1,\left[\frac{g-1}{3}\right], n\right)},
$$

where we denote the \pm subscript universally as $(-1)^{g+n+1}$ depending on the sign. Here we have also set $m=\left[\frac{19(g-1)}{3}\right]$ in (E.8). The space $Y^{\left(g, d_{B}\right)}$ is exactly the space of remaining holomorphic ambiguities at genus $g$, after we impose the boundary conditions at the conifold and orbifold loci, and use the exact formulas for base degrees up to $d_{B}$. Now we consider $g=9\left(d_{B}+1\right)$, then according to (C.10), (C.11), the dimension of the first linear space in the direct sum (E.10) is

$$
\operatorname{Dim}\left(V_{-}^{\left(1,3 d_{B}+2, d_{B}+1\right)}\right)=0 .
$$

The dimensions of the other spaces in (E.10) are smaller and also vanish, so we have $\operatorname{Dim}\left(Y^{\left(9\left(d_{B}+1\right), d_{B}\right)}\right)=0$, i.e. all holomorphic ambiguities can be fixed at genus $9\left(d_{B}+1\right)$. On the other hand, consider one genus higher at $g=9\left(d_{B}+1\right)+1$, then the dimension of the first linear space is computed similarly

$$
\operatorname{Dim}\left(V_{+}^{\left(1,3 d_{B}+3, d_{B}+1\right)}\right)=1>0 .
$$

so we have $\operatorname{Dim}\left(Y^{\left(9\left(d_{B}+1\right)+1, d_{B}\right)}\right) \geq \operatorname{Dim}\left(V_{+}^{\left(1,3 d_{B}+3, d_{B}+1\right)}\right)>0$, i.e. there are some unfixed holomorphic ambiguities.

Open Access. This article is distributed under the terms of the Creative Commons Attribution License (CC-BY 4.0), which permits any use, distribution and reproduction in any medium, provided the original author(s) and source are credited.

\section{References}

[1] M. Aganagic, V. Bouchard and A. Klemm, Topological strings and (almost) modular forms, Commun. Math. Phys. 277 (2008) 771 [hep-th/0607100] [INSPIRE].

[2] M. Alim and J.D. Lange, Polynomial structure of the (open) topological string partition function, JHEP 10 (2007) 045 [arXiv:0708.2886] [INSPIRE].

[3] M. Alim and E. Scheidegger, Topological strings on elliptic fibrations, Commun. Num. Theor. Phys. 08 (2014) 729 [arXiv:1205.1784] [INSPIRE].

[4] M. Alim, E. Scheidegger, S.-T. Yau and J. Zhou, Special polynomial rings, quasi modular forms and duality of topological strings, Adv. Theor. Math. Phys. 18 (2014) 401 [arXiv: 1306.0002] [INSPIRE].

[5] F. Benini, R. Eager, K. Hori and Y. Tachikawa, Elliptic genera of $2 D N=2$ gauge theories, Commun. Math. Phys. 333 (2015) 1241 [arXiv:1308.4896] [InSPIRE].

[6] M. Bershadsky, S. Cecotti, H. Ooguri and C. Vafa, Holomorphic anomalies in topological field theories, Nucl. Phys. B 405 (1993) 279 [hep-th/9302103] [INSPIRE].

[7] M. Bershadsky, S. Cecotti, H. Ooguri and C. Vafa, Kodaira-Spencer theory of gravity and exact results for quantum string amplitudes, Commun. Math. Phys. 165 (1994) 311 [hep-th/9309140] [INSPIRE]. 
[8] R.E. Borcherds, Automorphic forms with singularities on Grassmannians, Invent. Math. 132 (1998) 491 [alg-geom/9609022] [INSPIRE].

[9] J.H. Bruinier, G. van der Geer, G. Harder and D. Zagier, The 1-2-3 of modular forms, Springer, Berlin Germany (2008).

[10] N.C. Bizet, A. Klemm and D.V. Lopes, Landscaping with fluxes and the E8 Yukawa point in F-theory, arXiv: 1404.7645 [INSPIRE].

[11] W. Cai, M.-x. Huang and K. Sun, On the elliptic genus of three E-strings and heterotic strings, JHEP 01 (2015) 079 [arXiv:1411.2801] [INSPIRE].

[12] P. Candelas, A. Font, S.H. Katz and D.R. Morrison, Mirror symmetry for two parameter models. 2, Nucl. Phys. B 429 (1994) 626 [hep-th/9403187] [InSPIRE].

[13] T.M. Chiang, A. Klemm, S.-T. Yau and E. Zaslow, Local mirror symmetry: calculations and interpretations, Adv. Theor. Math. Phys. 3 (1999) 495 [hep-th/9903053] [InSPIRE].

[14] J. Choi, S. Katz and A. Klemm, The refined BPS index from stable pair invariants, Commun. Math. Phys. 328 (2014) 903 [arXiv:1210.4403] [InSPIRE].

[15] A. Dabholkar, S. Murthy and D. Zagier, Quantum black holes, wall crossing and mock modular forms, arXiv:1208.4074 [INSPIRE].

[16] B. de Wit and A. Van Proeyen, Potentials and symmetries of general gauged $N=2$ supergravity: Yang-Mills models, Nucl. Phys. B 245 (1984) 89 [INSPIRE].

[17] R. Dijkgraaf, G.W. Moore, E.P. Verlinde and H.L. Verlinde, Elliptic genera of symmetric products and second quantized strings, Commun. Math. Phys. 185 (1997) 197 [hep-th/9608096] [INSPIRE].

[18] N. Drukker, M. Mariño and P. Putrov, From weak to strong coupling in ABJM theory, Commun. Math. Phys. 306 (2011) 511 [arXiv:1007.3837] [InSPIRE].

[19] M. Eichler and D. Zagier, The theory of Jacobi forms, Progress in mathematics volume 55, Birkhäuser (1985).

[20] C. Faber and R. Pandharipande, Hodge integrals and Gromow-Witten theory, Invent. Math 139 (2000) 173 [math. AG/9810173].

[21] D.Z. Freedmann and A. van Proyen, Supergravity, Cambridge University Press, Cambridge U.K. (2012).

[22] W. Fulton, Introduction to toric varieties, Annals of Math Studies volume 131, Princeton University Press, Princeton U.S.A. (1993).

[23] O.J. Ganor, A test of the chiral $E_{8}$ current algebra on a $6-D$ noncritical string, Nucl. Phys. B 479 (1996) 197 [hep-th/9607020] [INSPIRE].

[24] R. Gopakumar and C. Vafa, M theory and topological strings. 2, hep-th/9812127 [INSPIRE].

[25] T.W. Grimm, A. Klemm, M. Mariño and M. Weiss, Direct integration of the topological string, JHEP 08 (2007) 058 [hep-th/0702187] [INSPIRE].

[26] M. Günaydin, A. Neitzke and B. Pioline, Topological wave functions and heat equations, JHEP 12 (2006) 070 [hep-th/0607200] [INSPIRE].

[27] B. Haghighat, A. Iqbal, C. Kozçaz, G. Lockhart and C. Vafa, M-strings, Commun. Math. Phys. 334 (2015) 779 [arXiv:1305.6322] [INSPIRE]. 
[28] B. Haghighat, A. Klemm, G. Lockhart and C. Vafa, Strings of minimal 6D SCFTs, Fortsch. Phys. 63 (2015) 294 [arXiv:1412.3152] [INSPIRE].

[29] B. Haghighat, A. Klemm and M. Rauch, Integrability of the holomorphic anomaly equations, JHEP 10 (2008) 097 [arXiv: 0809.1674] [INSPIRE].

[30] B. Haghighat, G. Lockhart and C. Vafa, Fusing E-strings to heterotic strings: $E+E \rightarrow H$, Phys. Rev. D 90 (2014) 126012 [arXiv:1406.0850] [InSPIRE].

[31] S. Hosono, Counting BPS states via holomorphic anomaly equations, hep-th/0206206 [INSPIRE].

[32] S. Hosono, A. Klemm, S. Theisen and S.-T. Yau, Mirror symmetry, mirror map and applications to Calabi-Yau hypersurfaces, Commun. Math. Phys. 167 (1995) 301 [hep-th/9308122] [INSPIRE].

[33] S. Hosono, A. Klemm, S. Theisen and S.-T. Yau, Mirror symmetry, mirror map and applications to complete intersection Calabi-Yau spaces, Nucl. Phys. B 433 (1995) 501 [hep-th/9406055] [INSPIRE].

[34] S. Hosono, M.H. Saito and A. Takahashi, Holomorphic anomaly equation and BPS state counting of rational elliptic surface, Adv. Theor. Math. Phys. 3 (1999) 177 [hep-th/9901151] [INSPIRE].

[35] S. Hosono, M.-H. Saito and A. Takahashi, Relative Lefschetz action and BPS state counting, math/0105148 [INSPIRE].

[36] M.-x. Huang, On gauge theory and topological string in Nekrasov-Shatashvili limit, JHEP 06 (2012) 152 [arXiv: 1205.3652] [INSPIRE].

[37] M.-x. Huang, Modular anomaly from holomorphic anomaly in mass deformed $\mathcal{N}=2$ superconformal field theories, Phys. Rev. D 87 (2013) 105010 [arXiv:1302.6095] [InSPIRE].

[38] M.-x. Huang, A.-K. Kashani-Poor and A. Klemm, The $\Omega$ deformed B-model for rigid $\mathcal{N}=2$ theories, Annales Henri Poincaré 14 (2013) 425 [arXiv:1109.5728] [INSPIRE].

[39] M.X. Huang, S. Katz, A. Klemm and D. Zagier, in progress.

[40] M.X. Huang and A. Klemm, Holomorphic anomaly in gauge theories and matrix models, JHEP 09 (2007) 054 [hep-th/0605195] [INSPIRE].

[41] M.X. Huang and A. Klemm, Holomorphicity and modularity in Seiberg-Witten theories with matter, JHEP 07 (2010) 083 [arXiv: 0902.1325] [INSPIRE].

[42] M.X. Huang and A. Klemm, Direct integration for general $\Omega$ backgrounds, Adv. Theor. Math. Phys. 16 (2012) 805 [arXiv: 1009.1126] [INSPIRE].

[43] M.X. Huang, A. Klemm and M. Poretschkin, Refined stable pair invariants for $E_{-}, M$ - and [p,q]-strings, JHEP 11 (2013) 112 [arXiv:1308.0619] [INSPIRE].

[44] M.X. Huang, A. Klemm and S. Quackenbush, Topological string theory on compact Calabi-Yau: modularity and boundary conditions, Lect. Notes Phys. 757 (2009) 45 [hep-th/0612125] [INSPIRE].

[45] A. Iqbal, C. Kozcaz and C. Vafa, The refined topological vertex, JHEP 10 (2009) 069 [hep-th/0701156] [INSPIRE].

[46] H. Jockers, A. Klemm and M. Romo, in preparation. 
[47] S. Kachru and C. Vafa, Exact results for $N=2$ compactifications of heterotic strings, Nucl. Phys. B 450 (1995) 69 [hep-th/9505105] [INSPIRE].

[48] M. Kaneko and D. Zagier, A generalized Jacobi theta function and quasimodular forms, in The moduli spaces of curves, R. Dijkgraaf et al. eds., Progress in Mathematics 129, Birkhäuser (1995).

[49] S. Katz, Gromov-Witten, Gopakumar-Vafa, and Donaldson-Thomas invariants of Calabi-Yau threefolds, in Snowbird lectures on string geometry, Contemporary Mathematics volume 401, K. Becker et al. eds., American Mathematical Society, Providence U.S.A. (2006).

[50] S.H. Katz, A. Klemm and C. Vafa, M theory, topological strings and spinning black holes, Adv. Theor. Math. Phys. 3 (1999) 1445 [hep-th/9910181] [InSPIRE].

[51] J. Kim, S. Kim, K. Lee, J. Park and C. Vafa, Elliptic genus of E-strings, arXiv:1411.2324 [INSPIRE].

[52] A. Klemm, M. Kreuzer, E. Riegler and E. Scheidegger, Topological string amplitudes, complete intersection Calabi-Yau spaces and threshold corrections, JHEP 05 (2005) 023 [hep-th/0410018] [INSPIRE].

[53] A. Klemm, W. Lerche and P. Mayr, K3 fibrations and heterotic type-II string duality, Phys. Lett. B 357 (1995) 313 [hep-th/9506112] [INSPIRE].

[54] A. Klemm, J. Manschot and T. Wotschke, Quantum geometry of elliptic Calabi-Yau manifolds, arXiv:1205.1795 [INSPIRE].

[55] A. Klemm, P. Mayr and C. Vafa, BPS states of exceptional noncritical strings, hep-th/9607139 [INSPIRE].

[56] A. Klemm, M. Poretschkin, T. Schimannek and M. Westerholt-Raum, to appear.

[57] K. Kodaira, On compact analytic surfaces, II, Ann. Math. 77 (1963) 563.

[58] K. Kodaira, On compact analytic surfaces, III, Ann. Math. 78 (1963) 1.

[59] M. Kontsevich, Product formulas for modular forms on $O(2, n)$ (after R.Borcherds), Astérisque 245 (1997) 41 [alg-geom/9709006].

[60] D. Krefl and J. Walcher, Extended holomorphic anomaly in gauge theory, Lett. Math. Phys. 95 (2011) 67 [arXiv:1007.0263] [INSPIRE].

[61] B.H. Lian and S.-T. Yau, Mirror maps, modular relations and hypergeometric series. II, Nucl. Phys. Proc. Suppl. 46 (1996) 248 [hep-th/9507153] [INSPIRE].

[62] J. Louis and K. Foerger, Holomorphic couplings in string theory, Nucl. Phys. Proc. Suppl. 55B (1997) 33 [hep-th/9611184] [INSPIRE].

[63] M. Mariño and G.W. Moore, Counting higher genus curves in a Calabi-Yau manifold, Nucl. Phys. B 543 (1999) 592 [hep-th/9808131] [INSPIRE].

[64] J.A. Minahan, D. Nemeschansky and N.P. Warner, Partition functions for BPS states of the noncritical $E_{8}$ string, Adv. Theor. Math. Phys. 1 (1998) 167 [hep-th/9707149] [INSPIRE].

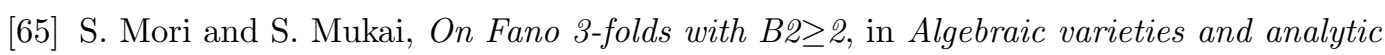
varieties, S. Iitaka ed., North-Holland publ. Co. (1983).

[66] G. Oberdieck and R. Pandharipande, Curve counting on $K 3 \times E$, the Igusa cusp form $\chi_{10}$ and descendent integration, arXiv:1411.1514 [INSPIRE]. 
[67] R. Pandharipande and R.P. Thomas, Stable pairs and BPS invariants, J. Amer. Math. Soc. 23 (2010) 267 [arXiv:0711.3899] [INSPIRE].

[68] M. Reid, The moduli space of 3-folds with $K=0$ may nevertheless be irreducible, Math. Ann. 278 (1987) 329.

[69] K. Sakai, Topological string amplitudes for the local half K3 surface, arXiv:1111.3967 [INSPIRE].

[70] A. Strominger, S.-T. Yau and E. Zaslow, Mirror symmetry is T duality, Nucl. Phys. B 479 (1996) 243 [hep-th/9606040] [INSPIRE].

[71] M.A.A. van Leeuwen, A.M. Cohen and B. Lisser, LiE, A Package for Lie Group Computations, Computer Algebra Nederland, Amsterdam The Netherlands (1992), http://wwwmathlabo.univ-poitiers.fr/ maavl/LiE/form.html.

[72] A. Van Proeyen, Vector multiplets in $N=2$ supersymmetry and its associated moduli spaces, hep-th/9512139 [INSPIRE].

[73] E. Witten, Quantum background independence in string theory, hep-th/9306122 [INSPIRE].

[74] S. Yamaguchi and S.-T. Yau, Topological string partition functions as polynomials, JHEP 07 (2004) 047 [hep-th/0406078] [INSPIRE].

[75] S.P. Zwegers, Mock theta functions, thesis, Utrecht, The Netherlands (2002). 